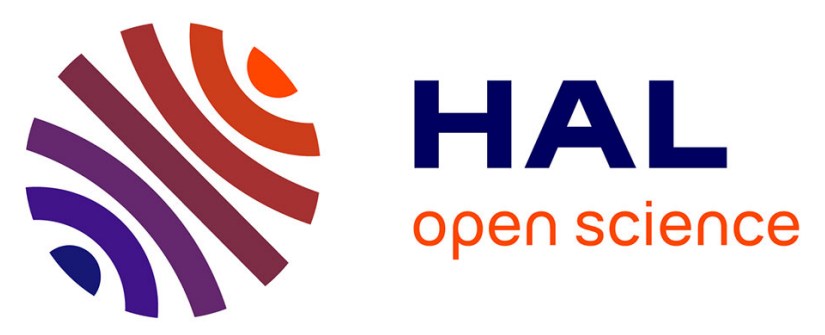

\title{
Self-determined motivation profiles among undergraduate students: A robust test of profile similarity as a function of gender and age
}

David Litalien, Nicolas Gillet, Marylène Gagné, Catherine Ratelle, Alexandre J.S. Morin

\section{To cite this version:}

David Litalien, Nicolas Gillet, Marylène Gagné, Catherine Ratelle, Alexandre J.S. Morin. Selfdetermined motivation profiles among undergraduate students: A robust test of profile similarity as a function of gender and age. Learning and Individual Differences, 2019, 70, pp.39-52. 10.1016/j.lindif.2019.01.005 . hal-03174109

\author{
HAL Id: hal-03174109 \\ https://hal.science/hal-03174109
}

Submitted on 18 Mar 2021

HAL is a multi-disciplinary open access archive for the deposit and dissemination of scientific research documents, whether they are published or not. The documents may come from teaching and research institutions in France or abroad, or from public or private research centers.
L'archive ouverte pluridisciplinaire HAL, est destinée au dépôt et à la diffusion de documents scientifiques de niveau recherche, publiés ou non, émanant des établissements d'enseignement et de recherche français ou étrangers, des laboratoires publics ou privés. 


\title{
Similarity as a Function of Gender and Age
}

\author{
David Litalien* \\ Faculté des sciences de l'éducation, Université Laval \\ Nicolas Gillet* \\ Department of Psychology, Université François-Rabelais \\ Marylène Gagné \\ Future of Work Institute, Faculty of Business and Law, Curtin University, Australia \\ Catherine F. Ratelle \\ Faculté des sciences de l'éducation, Université Laval, Canada \\ Alexandre J. S. Morin \\ Department of Psychology, Concordia University
}

This is the prepublication version of the following manuscript:

Litalien, D., Gillet, N., Gagné, M., Ratelle, C. F., \& Morin, A. J. S. (2019). Self-determined motivation profiles among undergraduate students: A robust test of profile similarity as a function of gender and age. Learning and Individual Differences, 70, 39-52.

(C2019. This paper is not the copy of record and may not exactly replicate the authoritative document published in Learning and Individual Differences. Please do not copy or cite without author's permission. The final article is available, upon publication, at: https://doi.org/10.1016/j.lindif.2019.01.005.

* Since the first two authors (D. L \& N. G.) contributed equally to the preparation of this article, their order was randomly determined.

Acknowledgements: The first author's work was supported by a research grant from the Fonds de Recherche du Québec - Société et Culture. 


\begin{abstract}
This study examines profiles of undergraduate students defined based on their types of behavioral regulation, as proposed by self-determination theory, as well as the similarity of these academic motivation profiles as a function of gender and age. This research also documents the implications of these profiles for students' vitality and investigates the role of maternal and paternal involvement, autonomy support, and warmth in predicting profile membership. A total of 1072 undergraduate students participated in this study (Mage $=22.7$ years; $58.4 \%$ female). To test for profile similarity, participants were divided into three age categories. Latent profile analyses revealed five distinct motivation profiles: Knowledge-Oriented, Controlled, Multifaceted, Unmotivated, and Hedonist profiles. These profiles, as well as their associations with the covariates, were similar across gender and age groups. Students' level of vitality varied across profiles and was higher in the Hedonist and Knowledge-Oriented profiles, whereas both parents' warmth predicted membership in profiles characterized by high levels of intrinsic motivation.
\end{abstract}

Keywords: academic motivation profiles, undergraduate students, gender, latent profile analyses, selfdetermination theory 
Self-determination theory (SDT; Deci \& Ryan, 2000; Ryan \& Deci, 2017) conceptualizes academic motivation as a series of distinct, yet complementary, forms of behavioral regulation that may coexist within students and play a role in the emergence of goal-directed behaviors. These types of regulation range from more autonomous forms of motivation, which characterize an engagement in activities driven by pleasure, volition, and choice, to more controlled forms of motivations, where activity engagement is driven by internal or external pressures. Numerous variable-centered studies have supported the existence of well-differentiated links between these forms of motivation and a series of personal and educational covariates (e.g., vitality, well-being, achievement, attainment; Gillet, Huyghebaert et al., 2017; Guay, Ratelle, \& Chanal, 2008; Mouratidis, Vansteenkiste, Lens, \& Sideridis, 2008). However, variablecentered research identifies average relations among variables occurring in a specific sample of students, and thus only provides limited information regarding the combined effects of these forms of motivation at the individual level. For instance, interaction effects involving more than three predictors (e.g., types of motivation) are almost impossible to interpret adequately using variable-centered analyses.

In contrast, person-centered analyses seek to identify subpopulations, referred to as profiles, characterized by distinct configurations on a set of interacting variables (Marsh, Lüdtke, Trautwein, \& Morin, 2009; Morin \& Wang, 2016), making them naturally suited to investigating the combined effects of behavioral regulations. Recently, person-centered studies began to look at how the different forms of motivation proposed by SDT combine with one another within specific profiles across multiple settings including education (Wang, Morin, Ryan, \& Liu, 2016), work (Gillet, Fouquereau, Vallerand, Abraham, \& Colombat, 2017), and sport (Gillet, Vallerand, \& Rosnet, 2009).

Unfortunately, prior investigations of students' academic motivation profiles (Baars \& Wijnia, 2018; Cox, Ullrich-French, \& Sabiston, 2013; Liu, Wang, Tan, Koh, \& Ee, 2009; Ullrich-French \& Cox, 2009; Ullrich-French, Cox, \& Cooper, 2016), have not yet considered all key facets of academic motivation, and have resulted in divergent conclusions regarding the relative importance of autonomous and controlled forms of motivation. In addition, to ascertain that profiles represent substantively meaningful subpopulations, it is important to systematically assess the extent to which these profiles generalize to distinct groups of participants (Marsh et al., 2009; Meyer \& Morin, 2016; Morin, Meyer, Creusier, \& Biétry, 2016). Person-centered evidence is cumulative in nature, and requires an accumulation of results obtained within distinct samples to differentiate the core subset of profiles that systematically emerges, the peripheral profiles that only emerges in specific situations, and the even less frequent set of profiles that simply reflects random sampling variations (e.g., Morin, 2016; Solinger, Van Olffen, Roe, \& Hofmans, 2013). Morin, Meyer et al. (2016) recently proposed a methodological framework designed to test the generalizability of the profiles identified across subpopulations of participants, making it possible to test for profile similarity within a single study and across meaningful subpopulations of participants.

In the present study, we rely on this person-centered framework to identify the configurations of behavioral regulations naturally occurring within specific profiles of students. Specifically, the present research extends the literature on undergraduate students' academic motivation profiles by (1) simultaneously considering all forms of motivation proposed by SDT, rather than relying on a reduced number of more global dimensions (e.g., autonomous versus controlled motivations); (2) assessing the three forms of intrinsic motivation proposed by Vallerand (1997), namely intrinsic motivation to know, intrinsic motivation to experience stimulation, and intrinsic motivation toward accomplishment (see also Carbonneau, Vallerand, \& Lafrenière, 2012), rather than relying on a global score of intrinsic motivation; (3) considering students' levels of amotivation, contrary to previous research which has tended to neglect this key facet of human motivation (e.g., Cox et al., 2013; Vansteenkiste, Sierens, Soenens, Luyckx, \& Lens, 2009); (4) systematically assessing the similarity (generalizability) of the profiles occurring across distinct groups of students formed on the basis of age and gender; (5) assessing the construct validity of the profiles in relation to one key educational covariate (i.e., vitality); and (6) considering the role of students' perceptions of their parents' behaviors (i.e., maternal and paternal involvement, autonomy support, and warmth) as possible determinants of motivation profiles.

\section{Self-Determination Theory}

According to SDT (Deci \& Ryan, 2000; Ryan \& Deci, 2017), students can be motivated for a variety of reasons. Intrinsic motivation represents volitional engagement in an activity for the pleasure and satisfaction that it affords. Vallerand (1997; Vallerand et al., 1992) underscored the importance of differentiating between three types of intrinsic motivation: (a) to know (i.e., engaging in an activity for the pleasure and satisfaction experienced while learning and exploring something new); (b) to accomplish (i.e., 
engaging in an activity for the pleasure derived from trying to surpass oneself and to improve one's skills), and (c) to experience stimulation (i.e., engaging in an activity for the pleasant sensations one derives from it). In education, studies have since corroborated this tripartite conceptualization of intrinsic motivation (e.g., Carbonneau et al., 2012), although a recent variable-centered meta-analysis reveals high correlations between these three subscales (Howard, Gagné, \& Bureau, 2017). Identified regulation refers to engagement in an activity that serves a personally-endorsed value or objective. Intrinsic motivation and identified regulation are conceptualized as autonomous forms of behavioral regulation. Introjected regulation refers to engagement in an activity driven by internal pressures, such as the avoidance of guilt and shame, or the pursuit of pride. External regulation refers to engagement in an activity that is controlled by external sources, such as rewards, punishments, or constraints. Introjected and external regulations are conceptualized as controlled forms of behavioral regulation. Finally, amotivation refers to the lack of motivation or intention toward the target behavior.

SDT does not conceptualize these types of motivation as mutually exclusive. Instead, these forms of motivation are proposed to coexist within individual students (Ratelle, Guay, Vallerand, Larose, \& Senécal, 2007), and to follow a self-determination continuum, ranging from intrinsic motivation to amotivation (Deci \& Ryan, 2000; Howard, Gagné, Morin, \& Forest, 2017). Still, as noted above, numerous studies have established the differential predictive validity of these forms of motivation for a variety of educational covariates (Guay et al., 2008; Litalien, Guay, \& Morin, 2015; Mouratidis et al., 2008). These studies have generally supported SDT in showing that autonomous forms of motivation tend to predict more positive outcomes than controlled forms of motivation and amotivation. Yet, some studies also showed that, contrary to expectations, controlled forms of motivation did not necessarily lead to negative outcomes. For instance, Vallerand et al. (1993) showed that introjected and external regulations toward school were positively related to concentration, the experience of positive emotions in the classroom, and performance. An interesting perspective on this question comes from emerging personcentered research showing that controlled forms of behavioral regulation may be associated with positive outcomes, but only when accompanied by similarly high levels of autonomous motivation (e.g., Gillet, Becker, Lafrenière, Huart, \& Fouquereau, 2017; Ratelle et al., 2007; Wang et al., 2016), underscoring the importance of studying the different forms of motivation in combination, rather than in isolation.

\section{Motivation Profiles}

Few person-centered studies of student motivation have been conducted in the educational area. Among these studies, some have identified profiles based on a combination of motivation types proposed by SDT and additional constructs (e.g., approach-avoidance goals: Smith, Deemer, Thoman, \& Zazworsky, 2014; social achievement goals: Mouratidis \& Michou, 2011), making it impossible to identify configurations of behavioral regulations in isolation from these additional dimensions. Among the studies that solely focused on motivation, many have relied on global dimensions (e.g., intrinsic and extrinsic motivation, Hayenga \& Corpus, 2010; autonomous and controlled motivation, Vansteenkiste et al., 2009), rather than distinguishing all forms of motivation proposed to be important in SDT. Still, when we consider these studies as well as those in which a broader set of behavioral regulations has been combined (e.g., Baars \& Wijnia, 2018; Boiché, Sarrazin, Grouzet, Pelletier, \& Chanal, 2008; Liu et al., 2009; Ullrich-French \& Cox, 2009; Wang et al., 2016), despite some variations, the results seem to converge on profiles characterized by: (a) high levels of autonomous motivation, and low levels of controlled motivation and amotivation (HAu-LC-LAm); (b) high levels of autonomous and controlled motivation, and low levels of amotivation (HAu-HC-LAm), (c) low levels of autonomous motivation, and high levels of controlled motivation and amotivation (LAu-HC-HAm), and (d) low to moderate levels of autonomous motivation, controlled motivation, and amotivation (LAu-LC-LAm). Some of these studies (Boiché et al., 2008; Wang et al., 2016) have also supported the importance of differentiating among more specific motivation types by revealing profiles characterized by distinct levels of introjected and external regulation. Still, none of these studies considered the potentially critical distinction among the three types of intrinsic motivation proposed by Vallerand (1997; Vallerand et al., 1992).

The first purpose of the present study was to identify undergraduate students' academic motivation profiles using LPA, while simultaneously considering all facets of academic motivation proposed to be relevant according to SDT. Despite the fact that none of the prior person-centered studies considered a complete set of behavioral regulations encompassing all three types of intrinsic motivation, extant empirical and theoretical knowledge allow us to propose the following hypotheses:

Hypothesis 1. Students' academic motivation should be characterized by a relatively small 
number (between four and six) of profiles.

Hypothesis 2. Students' motivation should be characterized by profiles matching the four routinely observed configurations: (1) HAu-HC-LAm, (2) LAu-LC-LAm, (3) HAu-LC-LAm, and (4) LAu-HC-HAm.

Hypothesis 3. Students' motivation should be characterized by at least one profile presenting diverging levels of introjected and external regulations (Boiché et al., 2008; Wang et al., 2016).

Research Question 1. Lacking prior empirical guidance in this area, we leave as an open question whether students' motivation would reveal profiles characterized by diverging levels of intrinsic motivation to know, to experience stimulation, and to accomplish.

\section{Gender and Age Similarity}

As noted by Morin, Meyer et al. (2016), it is critical to systematically assess the construct validity of person-centered solutions in order to ascertain that the extracted profiles of participants are meaningful in their own right and can be expected to generalize across samples. The importance of this construct validation process is intimately related to the technical characteristics of person-centered analyses, which make it hard to rule out the extraction of spurious profiles due to violations of the model's distributional assumptions (e.g., Bauer \& Curran, 2004), and the true added-value of a person-centered solution relative to a mathematically equivalent variable-centered solution (e.g., Steinley \& McDonald, 2007). For these reasons, many scholars have reinforced the fact that the only way to really support a substantive interpretation of profiles is to demonstrate that they meaningfully relate to covariates and can reliably be replicated across samples (e.g., Marsh et al., 2009; Morin, Meyer et al., 2016).

To better document the construct validity of the academic motivation profiles identified in the present study, we systematically examined the extent to which these profiles generalized to student subpopulations defined based on gender and age. These two variables were selected in light of previous research results showing that motivational processes are expected to vary as a function of these variables, making it highly relevant to assess whether obtained profiles would generalize to these student subpopulations. For instance, prior variable-centered studies showed that females tend to report higher levels of autonomous motivation than males (Bonneville-Roussy, Evans, Verner-Filion, Vallerand, \& Bouffard, 2017; Ratelle et al., 2007; Vallerand, Fortier, \& Guay, 1997), whereas others studies did not find gender differences on motivation types (Hui, Sun, Chow, \& Chu, 2011; Seghers, Vissers, Rutten, Decroos, $\&$ Boen, 2014). Additional research also suggests that females may be characterized by higher level of introjected regulation and lower levels of amotivation and external regulations relative to males (e.g., Ratelle et al., 2007; Vallerand et al., 1997). Previous person-centered research also tended to yield inconsistent results. Thus, despite tentative evidence that the profile structure may be similar across samples of males and females (Ullrich-French et al., 2016), additional results showed females to be overrepresented in profiles characterized by average levels of motivation across dimensions, or by low levels of autonomous motivation in the sport area (Cox et al., 2013; Ullrich-French et al., 2016). Gillet, Vallerand, and Paty (2013) found that men were underrepresented in a profile characterized by high levels of intrinsic motivation, moderate levels of identified regulation, and low levels of external regulation and amotivation, but overrepresented in a profile with high levels of intrinsic motivation, identified regulation, and external regulation, and low levels of amotivation. Yet, additional studies found no evidence in the gender composition of motivational profiles (Gillet et al., 2009; Gillet, Berjot, Vallerand, Amoura, \& Rosnet, 2012), reinforcing the importance of systematic tests of generalizability.

In terms of age, many studies investigated how the various types of academic motivation fluctuate over students' development. Among variable-centered studies, there is a consensus that intrinsic motivation tends to decrease as a function of age during the elementary and secondary school periods (e.g., Corpus, McClintic-Gilbert, \& Hayenga, 2009; Gottfried, Marcoulides, Gottfried, Oliver, \& Guerin, 2007; Lepper, Corpus, \& Iyengar, 2005). Researchers also generally found a decline on most types of motivation during the same developmental periods (Gillet, Vallerand, \& Lafrenière, 2012; Gnambs \& Hanfstingl, 2016; Martinek, Hofmann, \& Kipman, 2016), although these average tendencies differ across profiles of youths (Ratelle, Guay, \& Sénécal, 2004). During junior high school in Japan $\left(7^{\text {th }}\right.$ to $9^{\text {th }}$ grades), Nishimura and Sakurai's (2017) results showed a decrease in levels of intrinsic and identified regulations, but an increase in introjected and extrinsic regulations. These results clearly suggest the presence of age-related differences in levels of academic motivation among elementary or secondary school students.

To our knowledge, none of these studies looked at whether these age differences on motivation persisted after the entry into university. In particular, university studies are more likely to 
be specific to an actively chosen domain and non-mandatory, suggesting possible differences in the specific motivational configurations that might be observed among university students. In addition, university students are likely to be representative of a wider age range than earlier school levels. Thus, if academic motivation profiles do indeed differ as a function of age, then the age-related diversity of motivation profiles identified among University students is likely to be greater than that observed during earlier educational periods. For instance, older undergraduate students, who tend to have more extensive academic, work, or life experiences, could also display different forms of motivation profiles than younger students who directly transited into university from high school or college.

The present study aimed to provide preliminary responses to these questions. In order to test the generalizability of the motivation profiles across the different age groups represented in undergraduate University programs, we contrasted three distinct samples (17 to 20, 21 to 23, and 24 or more) of undergraduate university students. Rather than using age as a continuous predictor of profile membership, this categorization allow us to more systematically assess the similarity of the identified profiles (i.e., number, structure, within-profile variability, size, and relations with predictors and covariates) across age groups (Morin, Meyer et al., 2016). To reduce the arbitrariness of this categorization, these groups were determined on the basis of Arnett's (2000) theory of emerging adulthood. Thus, the 17 to 20 age category reflects the beginning of the emerging adulthood period (Arnett, 2000) and is more likely to include first-or second-year university students who took a direct path to university. The 21 to 23 age category reflects the middle of the emerging adulthood period and is more likely to correspond to students with slightly longer postsecondary experiences (academic or not) than those who took a direct path from high school. The last category ( 24 or more) refers to the end of the emerging adulthood period and to older adults. Student from this category are more likely to have more academic, work, or life experiences. These various level of experiences could affect the reasons why students undertake undergraduate courses.

Research Question 2. A key objective in the present study is thus to systematically assess whether the profiles would be similar across gender and age groups. However, in the absence of prior empirical guidance and diverging results regarding gender and age effects on motivation profiles, we leave as an open research question the extent, and nature, of this similarity.

\section{The Association between Students' Motivation Profiles and Vitality}

Prior research on academic motivation profiles has documented associations between students' profiles and a variety of covariates. Consistent with SDT predictions (Deci \& Ryan, 2000; Ryan \& Deci, 2017), previous studies (Cox et al., 2013; Liu et al., 2009) showed positive relations between a variety of desirable characteristics and profiles characterized by high levels of autonomous motivation but low levels of controlled motivation and amotivation, and negative relations between these same characteristics and profiles characterized by low levels of autonomous motivation coupled with higher levels of controlled motivation and amotivation. However, and contrary to predictions, additional studies showed that levels of controlled motivation apparently had no relation with these characteristics (i.e., cognitive disorganization in Boiché \& Stephan, 2014; achievement in Ratelle et al., 2007) when levels of autonomous motivation were high, thus calling into question theoretical predictions alluding to the undesirability of controlled types of motivation (Ryan \& Deci, 2017).

In the present study, we focus on general vitality as a key covariate of students' motivational profiles given mounting research evidence supporting the role of students' vitality as a key predictor of academic success (e.g., Coffey, Wray-Lake, Mashek, \& Branand, 2016). This decision to focus on general levels of vitality is aligned with the results from previous studies conducted in the educational (e.g., Burton, Lydon, D’Alessandro, \& Koestner, 2006; Litalien, Guay, \& Morin, 2015) or other areas (religion, Ryan, Rigby, \& King, 1993; weight-loss treatment, Ryan \& Frederick, 1997) which have documented the link between domain-specific measures of motivation and general indicators of wellbeing. Furthermore, Ryan and Deci (2017, p. 256) note that vitality is "perhaps the most general characteristic of a fully functioning person". Indeed, students will invest a large amount of their time in their studies, leading us to expect that the way they approached these studies is likely to have an impact on their global levels of vitality. All of these considerations led us to expect students' motivation profiles to be differentially related to vitality, leading to the following hypotheses:

Hypothesis 4. We expect students' profiles characterized by high levels of autonomous motivation and low levels of amotivation, regardless of their levels of controlled motivation (e.g., HAu-HC-LAm and HAu-LC-LAm profiles), to report the highest levels of vitality. 
Hypothesis 5. We expect students' profiles characterized by high levels of amotivation and low levels of autonomous motivation, regardless of their levels of controlled motivation (e.g., LAuHC-HAm), to report the lowest levels of vitality.

Research Question 3. In the absence of prior guidance, we left as an open research question whether associations between motivational profiles and vitality would differ as a function of age and gender. However, we have no empirical or theoretical reason to expect differences.

\section{Predictors of Motivation Profiles}

Documenting which motivational profile (e.g., HAu-HC-LAm or HAu-LC-LAm profiles) is the most desirable from a covariate perspective (i.e., associated with the highest levels of vitality) is only a first step. In order to achieve a clear understanding of students' motivational profiles, a next logical step is to identify what predicts membership into these motivational profiles. Surprisingly, little research has been conducted to investigate the predictors of motivation profiles in education. Among the few exceptions, students' motivation profiles have been associated with the level of satisfaction of their needs for competence, relatedness, and autonomy (Liu et al., 2009), and to their perceptions of the teaching climate (teacher autonomy support, structure, and involvement), as a key source of satisfaction of these needs (Vansteenkiste et al., 2009). The present study focuses on another key source of need satisfaction for students: students' perceptions of their mother and father involvement, autonomy support, and warmth, as possible predictors of likelihood into the various motivational profiles.

Parental autonomy support refers to behaviors through which mothers and fathers recognize their offspring's unique and volitional nature (Grolnick, 2003). Parental involvement is observed when mothers and fathers allocate important resources to their offspring. These not only include material resources (e.g., purchasing books and dictionaries, organizing a study area with a proper work desk), but also the allocation of time and support to the student. Finally, parental warmth refers to the allocation of more emotional resources to the student, such as like showing interest for what is occurring in their life and the demonstration of warmth and acceptance (see Pomerantz, Kim, \& Cheung, 2012; Ryan \& Deci, 2017).

Based on SDT (Grolnick, Deci, \& Ryan, 1997), in which these parental characteristics are explicitly proposed to contribute to the satisfaction of youth psychological needs, it would be logical to expect mother and father involvement, autonomy support, and warmth to foster autonomous forms of motivation and to decrease controlled forms of motivation and amotivation. Indeed, these parental behaviors have been repeatedly found to predict important academic outcomes such as students' psychological need satisfaction in school, and a variety of desirable motivational outcomes (Guay et al., 2008; Pomerantz et al., 2012; Ratelle \& Duchesne, 2017). To the best of our knowledge, the current study represents the first attempt to examine the role of parental need supporting behaviors in the prediction of membership in motivation profiles. Yet, despite the lack of person-centered evidence, abundant variablecentered evidence thus allow us to propose the following hypotheses:

Hypothesis 6. Parental involvement, autonomy support, and warmth should be positively related to the likelihood of membership into the profile characterized by high levels of autonomous motivation, and low levels of controlled motivation and amotivation (HAu-LC-Lam).

Hypothesis 7. Parental involvement, autonomy support, and warmth should be negatively related to the likelihood of membership into the profile characterized by high levels of amotivation (LAuHC-HAm).

Research Question 4. This study is the first to consider the characteristics of both mothers and fathers in relation to students' motivation profiles. Lacking prior empirical and theoretical guidance in this regard, we leave as an open question possible differences related to the relative importance and contribution of maternal and paternal behaviors.

Research Question 5. Based on limited research suggesting that exposure to parental behaviors may (e.g., Fousiani et al., 2014) or may not (Vallerand et al., 1997) differ as a function of youth gender and age, we also leave as an open research question the extent to which these relations will generalize to the various age- and gender- differentiated subsamples considered here.

\section{Participants and Procedures}

\section{Method}

A total of 1072 undergraduate students from an English-speaking Canadian university participated voluntarily to this study in exchange for extra credits toward an introductory organizational behavior course. Their mean age was 22.7 years $(S D=4.6$; range $=17$ to 48$)$ and $58.4 \%$ of them were female. For similarity tests conducted as a function of age, three age groups are considered: (a) 17 to 20 (38.8\%); (b) 
21 to $23(36.1 \%)$, (c) 24 or more $(25.1 \%)$. This research project was approved by the University research ethics committee and was conducted in accordance with the guidelines and principles for human subjects of the Canadian Interagency Advisory Panel on Research Ethics.

\section{Measures}

Academic motivation. The 28 items from the Academic Motivation Scale (AMS; Vallerand et al., 1992, 1993) were used to assess seven dimensions (4 items each) of academic motivation. Following a general question, Why do you go to college?, participants were asked to indicate to what extent each item corresponds to their reasons for going to college. The seven dimensions were: (a) intrinsic motivation to know ( $\alpha=.74$; e.g., "Because I experience pleasure and satisfaction while learning new things"); (b) intrinsic motivation to experience stimulation $(\alpha=.87$; e.g., "For the pleasure that I experience when I feel completely absorbed by what certain authors have written"); (c) intrinsic motivation to accomplish ( $\alpha=$. 80; e.g., "For the satisfaction I feel when I am in the process of accomplishing difficult academic activities"); (d) identified regulation ( $\alpha=.79$; e.g., "Because I believe that a few additional years of education will improve my competence as a worker"); (e) introjected regulation ( $\alpha=.85$; e.g., "To prove to myself that I am capable of completing my university degree"); (f) external regulation ( $\alpha=.82$; e.g., "In order to have a better salary later on"); and (g) amotivation ( $\alpha=.96$; e.g., "I can't see why I go to university and frankly, I couldn't care less"). Participants were asked to rate each item using a seven-point Likert scale $(1=$ does not correspond at all, $7=$ corresponds exactly). For a review of the psychometric properties of the AMS, see Guay, Morin, Litalien, Valois, and Vallerand (2015).

Vitality. The 7 items from the Subjective Vitality Scale (Ryan \& Frederick, 1997) were used to assess vitality at the general level ( $\alpha=.88$; e.g., "I have been feeling very alert and awake"). Each item was rated on a seven-point Likert scale $(1=$ not at all true, $7=$ very true $)$.

Perceptions of Parents. The 21 items of the Perceptions of Parents Scale (Robbins, 1994) were used to assess students' perceptions of both their mother's and father's involvement ( 6 items, $\alpha=.828$ for mothers and .86 for fathers; e.g., "My mother/father finds time to talk with me"); autonomy support (9 items, $\alpha=.85$ for mothers and .86 for fathers; e.g., "My mother/father helps me to choose my own direction"), and warmth ( 6 items, $\alpha=.88$ for mothers and .89 for fathers; e.g., "My mother/father clearly conveys his love for me"). Students' were asked to respond to each item while referring to the person whom they considered to be their mother and father figures. Students with no father or mother figures were instructed to leave the relevant section empty. Each item was rated on a seven-point Likert scale $(1=$ not at all true, $7=$ very true $)$.

\section{Latent Profile Analyses}

\section{Analyses}

Motivational profiles were first identified separately in each age- and gender- subsample using the robust Maximum Likelihood estimator available in Mplus 7.3 (Muthén, \& Muthén, 2014) and full information maximum likelihood estimation to handle the missing data present at the item level on motivation ratings ( $<2.4 \%$; Enders, 2010; Graham, 2012). Models including one to eight latent profiles were estimated using 5000 random sets of start values, 100 iterations, with the 200 best solutions retained for final stage optimization (Hipp \& Bauer, 2006). These analyses were based on factor scores (estimated with a mean of 0 and a standard deviation of 1 in the total sample) reflecting each of the seven motivation scales. These factor scores were saved from measurement models reported in the initial section of the online supplements. These models supported the factor validity and measurement invariance of the AMS ratings across gender and age groups. Although factor scores do not explicitly control for measurement errors the way latent variables do, they provide a partial control for measurement errors by giving more weight to items presenting lower levels of measurement errors (Skrondal \& Laake, 2001). Furthermore, factor scores are able to preserve the nature of the underlying measurement structure (e.g., invariance) better than scale scores. For a more extensive discussion of factor scores, see Morin, Meyer et al. (2016).

In the estimation of the latent profiles, means and variances of the profile indicators (i.e., the motivation factor scores) were freely estimated in all profiles (Diallo, Morin, \& Lu, 2016; Peugh \& Fan, 2013). Traditional LPA assume that the presence of the profiles will be sufficient to explain all of the correlations between the indicators (i.e., motivation types). Morin and Marsh (2015) highlighted the unrealism of this requirement when profile indicators can be assumed to reflect an overarching construct, and proposed a factor mixture approach as a way to relax this assumption. In line with recent research evidence showing that motivation ratings do indeed tap into a global construct representing the underlying continuum of motivation (Howard, Gagné, Morin, et al., 2017; Litalien et al., 2017), we adopted Morin 
and Marsh's (2015) factor mixture approach in this study. Specifically, this approach incorporates a continuous latent factor, specified to be invariant across profiles, to control for students' global level of motivation. Morin and Marsh (2015) have shown this approach to result in the estimation of profiles presenting clearer structural differences on specific motivation types. Preliminary analyses (available upon request from the first author), supported the superiority of this approach over that of classical LPA models.

To determine the optimal number of profiles in the data, it is important to consider the substantive meaning and theoretical conformity of the profiles (Marsh et al., 2009), the statistical adequacy of the solution (Bauer \& Curran, 2004), and the multiple statistical indicators available to help in this decision (McLachlan \& Peel, 2000). Among these statistical indicators, we report the Akaike Information Criterion (AIC), the Bayesian information criterion (BIC), the Consistent AIC (CAIC), the sample-adjusted BIC (ABIC), the adjusted Lo, Mendell, and Rubin's (2001) likelihood ratio test (aLMR), and the Bootstrap Likelihood Ratio Test (BLRT). Results from simulation studies indicate that the CAIC, BIC, ABIC, and BLRT are particularly effective (e.g., Diallo et al., 2016; Nylund, Asparouhov, \& Muthén, 2007; Tofighi $\&$ Enders, 2008). A lower value on the AIC, CAIC, BIC and ABIC suggests a better-fitting model. Both the aLMR and BLRT compare a $k$-profile model with a $k$ - 1 -profile model. A statistically significant $p$ value indicates that the $k-1$-profile model should be rejected in favor of a $k$-profile model. However, since these tests are all variations of tests of statistical significance, the class enumeration procedure can still be heavily influenced by sample size (Marsh et al., 2009). That is, with sufficiently large samples, these indicators may keep improving with the addition of latent profiles without reaching a minimum. In these cases, information criteria should be graphically presented through "elbow plots" illustrating the gains associated with additional profiles (Morin, Maïano et al., 2011). In these plots, the point after which the slope flattens indicates the optimal number of profiles in the data. Finally, the entropy indicates the precision with which the cases are classified into the profiles (varying from 0 to 1), but should not be used to determine the optimal number of profiles.

\section{Multiple Group Comparisons}

Multiple group LPA (the KNOWNCLASS function was used to identify gender or age groups ${ }^{1}$ ) were conducted to assess the similarity of the profile solutions identified across gender and age groups, following the sequence proposed by Morin, Meyer et al. (2016). Through this sequence, models including equality constraints across subgroups are compared to previous, less restricted models forming the sequence, using the information criteria (AIC, CAIC, BIC, ABIC) described above. Lower values on at least two of those criteria are taken to suggest that the equality constraints imposed across samples are supported by the data (Morin, Meyer et al., 2016).

The first step of this sequence is the verification of whether the same number of profiles can be identified across groups (i.e., the configural similarity). When this is the case, a multiple group model can be estimated, and equality constraints can be progressively integrated. In the second step, the structural similarity of the profiles is tested by including equality constraints across groups (i.e., gender or age groups) on the within-profile means of the various indicators (i.e., types of motivation). Evidence of structural similarity is sufficient to argue that the nature of the profiles is the same for males and females, which represents a prerequisite for the subsequent tests. If structural similarity holds, then the third step tests the dispersion similarity of the profiles by including equality constraints across groups on the withinprofile variances of the indicators. LPA does not assume that all individuals within a profile share the exact same configuration of indicators, but allow for within-profile variability. Testing for dispersion similarity thus verifies whether the profiles are more or less homogenous across groups, or members of specific groups present higher levels of within-profile variability than others. Fourth, the distributional similarity of the profiles is tested by constraining the relative size of the profiles to be equal across groups. This test verifies whether the proportion of students corresponding to each profile is similar across groups.

\section{Associations Between Profile Membership, Vitality, and the Predictors}

Scores on the predictors and vitality were also factor scores from measurement models reported in the online supplements, which supported the factor validity and measurement invariance of these constructs across gender and age groups. Correlations among all variables are reported in Table 1, together with model-based estimates of composite reliability calculated using with McDonald's (1970) omega $(\omega)$

\footnotetext{
${ }^{1}$ Current implementations of tests of profile similarity require the reliance on a multigroup approach, which forced us to rely on a categorization of participants age. This categorization was determined based on Arnett's (2001) emerging adulthood theory $(17-20 ; 21-23 ; 24+)$.
} 
coefficient. These estimates were all fully satisfactory $(\omega=.68$ to $.95 ; M=.82)$.

Vitality was incorporated into the final latent profile solution. The multivariate delta method (Raykov \& Marcoulides, 2004) was used to test mean-level differences across all pairs of profiles. Following the incorporation of this variable to the model, we proceeded to tests of explanatory similarity by constraining the within-profile mean of vitality to equality across gender and age groups.

Predictors were also integrated to the most similar model from the previous sequence through a multinomial logistic regression. In multinomial logistic regressions, each predictor has $k-1$ (with $k$ being the number of profiles) complementary effects for each possible pairwise comparison of profiles. These regression coefficients reflect, for each unit increase in the predictor, the increase that can be expected in the log odds of the outcome (i.e., the probability of membership in one profile versus another). We also report odds ratios (OR), reflecting the change in likelihood of membership in a target profile versus a comparison profile associated for each unit of increase in the predictor. Following the incorporation of the predictors, we proceeded to tests of predictive similarity by constraining these predictions to equality across gender and age groups.

A strong assumption of latent profile models including covariates and predictors is that the nature of the profiles should remain unaffected by the inclusion of these additional variables (Diallo, Morin, \& Lu, 2017; Morin, Morizot, Boudrias, \& Madore, 2011). Observing such a change calls into question the assumption that the causal ordering is from the predictors to the profiles, and from the profiles to the covariates. To ensure that this did not happen, all models including the predictors and vitality were estimated using manual three-step approach described by Asparouhov and Muthén (2014; also see Diallo \& Lu, 2017; McLarnon \& O’Neil, 2018; Morin \& Litalien, 2017). Still, despite this strategy, it was not possible to incorporate predictors related to the mother and father into the same model due to the nonconvergence of models including both parents. For this reason, models involving predictors related to the mother and father were estimated separately. Annotated input codes for the LPA models estimated in the present study are provided at the end of the online supplements, together with instructions for the application of this three-step approach to multiple group models.

\section{Results}

The goodness-of fit indicators for the solutions including 1 to 8 latent profiles estimated separately in each gender and age group are respectively reported in Tables S4 and S5 of the online supplements. For each group, the AIC, ABIC, and BLRT kept on improving with the addition of latent profiles to the data without ever reaching a minimum. The CAIC and the BIC similarly failed to reach a minimum among females, as well as participants aged between 17 and 20 years. The BIC also failed to reach a minimum among participants aged 21 to 23. In other groups however, the CAIC and the BIC respectively converged on solutions varying between five and seven profiles. Finally, the aLMR, an indicator with a known tendency for underextraction, suggested solutions varying between two and three profiles across subgroups. Taken together, these indicators provide limited information to guide the selection of the optimal number of latent profiles. To complement this information, we relied on a graphical representation of the values of the information criteria.

These elbow plots are reported in Figures S1 to S5 of the online supplements, and reveal that the decrease in the value of most indices, in most groups, reaches a plateau at either four or five profiles, although the true inflection point remains unclear, and that the decrease in the value of the statistical indicators observed before reaching this plateau remains substantial (Raftery, 1995). Examination of the 5profile solution, and of the bordering 4- and 6-profile solutions, showed that all solutions were fully proper, statistically. More importantly, these alternative solutions revealed profiles with the same general shape across groups, with initially observed differences disappearing when profiles were added to the solution, thus providing initial support to the configural similarity of the solution. This examination also revealed that adding a fifth profile always resulted in the addition of a well-defined qualitatively distinct and theoretically meaningful profile, whereas adding a sixth profile often resulted in the arbitrary division of an existing profile into ones differing only quantitatively. The 5-profile solution was thus retained for each gender and age group, providing a good level of classification accuracy with an entropy value ranging from .88 for females to .92 for the 21 to 23 -year-old group. This result is consistent with Hypothesis 1 .

\section{Gender and Age Similarity (Structural, Dispersion, and Distributional) of the Profiles}

The goodness-of fit indices associated with the gender and age similarity models are reported in Table 2. For both gender and age groups, the sequence of similarity tests yielded the same conclusions. This sequence started with the estimation of multiple-group 5-profile model of configural similarity. From 
this model, we first estimated a model of structural similarity. For both gender and age solutions, this model resulted in a lower value on the CAIC, BIC, and ABIC, suggesting that the structure of the profiles can be considered to be similar across gender and age groups. Second, we estimated a model of dispersion similarity, which resulted in lower values on the CAIC, BIC, and ABIC for both models (and also in lower values on the AIC in the age model), thus supporting the dispersion similarity of the profiles. Finally, we estimated a model of distributional similarity, in which the relative size of the profiles was constrained to be equivalent across gender and across age groups. This model resulted in lower values on the CAIC and BIC for both models (and also in lower values on the AIC and ABIC in the age model), supporting the distributional similarity of the profiles. In sum, these results address Research Question 2 by showing that the final set of latent profiles can be considered to be fully equivalent across all age and gender groups. The 5-profile model of distributional similarity was thus retained for interpretation and for the next stages.

This 5-profile final solution is illustrated in Figure 1. For greater precision, the exact within-profile means and variance for each type of motivation are reported in Table S6 of the online supplements. Profile 1 is characterized by moderately high levels of intrinsic motivation to know, low levels of amotivation, and average levels on the other types of motivations. This suggests that the desire to acquire knowledge through university study is particularly important in this profile, which we thus labelled "KnowledgeOriented". This profile represents $17.6 \%$ of the total sample ${ }^{2}$. In contrast, the second profile is characterized by moderately high levels of controlled motivation (introjected and external regulations), moderately low levels of amotivation, and average levels on the autonomous motivations (intrinsic motivation to know, to experience stimulation, and to accomplish, and identified regulation). We labelled this profile "Controlled", which appears to be relatively frequent, representing $26.0 \%$ of the sample.

Profile 3 is characterized by moderately high to very high levels on most types of motivation, low levels of amotivation, and average levels of intrinsic motivation to experience stimulation. This "Multifaceted" profile represent $15.0 \%$ of the students. In contrast, Profile 4 presents the opposite pattern of scores, being characterized by low to moderately low scores on most types of motivation, but a moderately high score of amotivation, and an average score of intrinsic motivation to experience stimulation. This "Unmotivated" profile was also relatively frequent, representing $25.4 \%$ of the sample. Finally, Profile 5 is characterized by a very high level of intrinsic motivation to experience stimulation and amotivation, moderately high levels of identified regulation, average levels of intrinsic motivation to know, intrinsic motivation to accomplish, and external regulation, and a very low level of introjected regulation. This result supports Hypothesis 3. We use the label "Hedonist" to describe this profile, which was rather unexpected. On the one hand, these students seem strongly stimulated by the pleasure they experience when reading about interesting authors and communicating their ideas (i.e., very high levels of intrinsic motivation to experience stimulation). On the other hand, they also appear to feel that attending college only offers limited opportunities for such stimulation (i.e., very high level of amotivation), despite acknowledging that higher education can contribute to their socio-professional integration (i.e., moderately high levels of identified regulation). This profile represents $16.0 \%$ of the students. Only two out of the five obtained profiles were similar to those mentioned in Hypothesis 2 (LAu-HC-Ham: Controlled; HAu-HCLam: Multifaceted), thus only providing partial support to this hypothesis. However, in response to our Research Question 1, it is interesting to note that all profiles identified here were characterized by diverging levels of intrinsic motivation to know, to experience stimulation, and to accomplish.

\section{Explanatory Similarity of the Profiles}

Vitality was added as a covariate to the final model of distributional similarity described earlier. We first estimated models in which the within-profile levels of vitality were freely estimated across gender and age groups, and contrasted these models with models in which these levels were constrained to be equal across gender or age groups (i.e., explanatory similarity). As shown in Table 2, the models in which the within-profile levels of vitality were constrained to equality across gender resulted in lower CAIC and $\mathrm{BIC}$ values, whereas those in which these levels were constrained to equality across age groups resulted in lower AIC, CAIC, BIC, and ABIC values. This results supports the explanatory similarity of the model, thus answering our Research Question 3 with evidence of generalizability across samples. Specific levels of vitality observed in each profile, expressed in standard deviations units, are 0.30 for Profile 1 (Knowledge-Oriented), -0.14 for Profile 2 (Controlled), 0.12 for Profile 3 (Multifaceted), -0.26 for Profile

\footnotetext{
${ }^{2}$ Supplementary analyses in which age was operationalized as a continuous predictor of profiles' membership were also realized, and supported the lack of age effects on profile membership.
} 
4 (Unmotivated), and 1.95 for Profile 5 (Hedonist). Results further showed that levels of vitality were statistically higher in Profile 5 (Hedonist) in comparison to all other profiles. No significant difference was observed between Profiles 1 (Knowledge-Oriented) and 3 (Multifaceted). However, these two profiles presented a higher level of vitality than Profiles 2 (Controlled) and 4 (Unmotivated), which did not differ statistically from one another. Detailed results from these comparisons are reported in Table $\mathrm{S} 7$ of the online supplements. These results partly support Hypotheses 4 and 5, respectively showing higher vitality levels to be associated in the profiles characterized by high level of autonomous motivation and low level of amotivation (Multifaceted and Knowledge-Oriented), and lower vitality levels to be associated with the profile showing an opposite pattern (Unmotivated).

\section{Predictive Similarity of the Profiles}

Predictors of profile membership, that is perceptions of maternal and paternal involvement, autonomy support, and warmth, were added to the final model of distributional similarity. We first estimated models (one for the mother, and one for the father) in which the associations between the predictors and the probability of profile membership was freely estimated across gender or age groups, and contrasted these models with models in which these relations were constrained to equality across gender or age groups (i.e., predictive similarity). As shown in Table 2, the models of predictive similarity across gender or age groups, for both sets of predictors resulted in lower values for the AIC, CAIC, BIC, and $\mathrm{ABIC}$ and were thus retained for interpretation. This results supports the predictive similarity of the model, thus answering our Research Question 5 with evidence of generalizability across samples.

Results from the multinomial logistic regressions estimated in the model of predictive similarity are reported in Table 3. For mothers, only warmth predicted profile membership. More precisely, higher levels of perceived maternal warmth increased the likelihood of membership into Profile 1 (KnowledgeOriented) in comparison to any other profiles, as well as decreased the probability of belonging to Profile 4 (Unmotivated) relative to Profiles 3 (Multifaceted) and 2 (Controlled). For fathers, all dimensions presented some levels of association with the likelihood of membership into the various profiles. More precisely, higher levels of perceived paternal involvement were associated with a greater likelihood of membership into Profile 4 (Unmotivated) relative to Profiles 1 (Knowledge-Oriented) and 2 (Controlled). In contrast, higher levels of perceived paternal autonomy support were associated with a greater likelihood of membership into Profile 3 (Multifaceted) in comparison to Profile 5 (Hedonist). Finally, higher levels of perceived paternal warmth were associated with a greater likelihood of membership into Profile 1 (Knowledge-Oriented) in comparison to Profiles 2 (Controlled) and 4 (Unmotivated), and into Profile 3 (Multifaceted) in comparison to Profile 4 (Unmotivated). Results in regard to parental behavior are mixed and only provided partial support to Hypotheses 6 and 7, which respectively proposed that parental involvement, autonomy support, and warmth should be positively related to the likelihood of membership into profiles characterized by high levels of autonomous motivation and low levels of controlled motivation and amotivation, and negatively related to profiles showing an opposite pattern. In response to our Research Question 4, they also support the idea that the identified relations differ across mothers and fathers.

\section{Students' Motivation Profiles}

\section{Discussion}

The first purpose of this study was to identify university students' profiles based on their configuration of motivation types proposed by SDT. Our results revealed that five profiles best represented undergraduate students' academic motivation, thus supporting Hypothesis 1, which suggested that a between four and six motivational profiles would be observed. The Controlled (i.e., moderately high levels of controlled motivations, average levels of autonomous motivations, and low levels of amotivation) and Multifaceted (moderately to high levels on most types of motivation, and low levels of amotivation) profiles were aligned with Hypothesis 2, respectively corresponding to the LAu-HC-Ham and HAu-HCLAm profiles identified in prior studies relying on a less extensive set of behavioral regulations (Boiché et al., 2008; Ratelle et al., 2007; Vansteenkiste et al., 2009). Our results also revealed three additional profiles presenting less similarity to the remaining profiles expected according to Hypothesis 2 (LAu-LC-LAm and HAu-LC-Lam profiles). Yet, it is noteworthy that two of these profiles were still observed in some previous studies, albeit less commonly than those covered in Hypothesis 2 (Ullrich-French \& Cox, 2009; Cannard, Lannegrand-Willems, Safont-Mottay, \& Zimmermann, 2016): Unmotivated (low levels of autonomous and controlled motivation, and high levels of amotivation) and Knowledge-Oriented (high levels of intrinsic motivation to know, low levels of amotivation, and average levels on the other types of 
motivations). In addition, despite the fact that it does not fully match the expected HAu-LC-Lam profile as it was characterized by close to average levels of controlled motivation and of two out of three types of intrinsic motivation, the Knowledge-Oriented profile remains dominated by a high level of intrinsic motivation (to know) and by a lack of amotivation. This profiles thus shares important similarities with the arguably most desired motivational state according to SDT (González et al., 2012) and does match the results from at least some of the prior studies (Cannard et al., 2016).

The Hedonist profile was unexpected, as it simultaneously presented high levels of amotivation coupled with equally high levels of intrinsic motivation to experience stimulation. These students, while being motivated to seek intellectual stimulation as part of their learning curriculum, seem to find that true possibilities to experience such absorbing and stimulating readings, discussions, and courses are far less frequent than what they would desire. A plausible explanation is that introduction courses like the one in which participants were recruited are often given to first-year undergraduate students, who generally tend to be exposed to broadly defined generic courses designed to establish the foundations of the program. As such, these students may feel being exposed to many courses that fail to truly capture their interest, while still being able to attend a small number of possibly optional courses which better match their expectations and desire to go beyond the more basic concepts covered in the majority of introductory courses. Clearly, these various possibilities would need to be more thoroughly considered in future research, which would be needed to assess whether this Hedonist profile will be replicated when considering new and more diversified samples of students.

It is noteworthy that we were able to identify these less common profiles, as well as a total set of five profiles. In contrast, prior research on academic motivation has typically found only three (Boiché et al., 2008; Ratelle et al., 2007) to four (González et al., 2012; Liu et al., 2009; Vansteenkiste et al., 2009) profiles. This greater level of precision supports the value of relying on a finer-grained representation of academic motivation incorporating specific forms of motivation (i.e., seven types) rather than simply focusing on the two global dimensions of autonomous and controlled motivations. Indeed, in line with our expectations (Hypothesis 3), we identified a profile showing diverging levels of introjected (very low) and external (average) regulations (Hedonist profile). In response to our first research question, four of the five profiles were characterized by different scores on the three forms of intrinsic motivation (to know, to experience stimulation, and to accomplish). Thus, in addition to the Knowledge-Oriented and Hedonist profiles which were mainly characterized by one specific type of intrinsic motivation, the levels of intrinsic motivation to experience stimulation were notably lower than those of the other types of intrinsic motivation in the Multifaceted profile, whereas the opposite was true in the Unmotivated profile.

These results support the added value of adopting a finer-grained representation of academic motivation (Ratelle et al., 2007). The added precision of our results may also stem from methodological differences (e.g., LPA rather than cluster analyses, and relying on factor scores providing a partial control for measurement errors). However, additional research using LPA and distinguishing the three types of intrinsic motivation proposed by Vallerand (1997) is needed in order to increase the generalizability of the present findings, especially in regard to less common profiles such as the Hedonist one.

To better document the construct validity of the five academic motivation profiles identified in the present study, we also examined the extent to which they would generalize to subpopulations defined on the basis of gender and age. Indeed, prior variable-centered studies showed significant differences in students' motivation levels as a function of these variables (e.g., Bonneville-Roussy et al., 2017; Lepper et al., 2005). Similarly, previous person-centered research suggested some gender differences in the composition of motivation profiles (e.g., Cox et al., 2013; Gillet et al., 2013). However, when relying on a systematic quantitative procedure aiming to precisely assess profile similarity (Morin, Meyer et al., 2016), our findings address our second research question by revealing that the five latent profiles identified in the present study could be considered to be fully equivalent in shape, within-profile variability, and size, across all age and gender groups. These results are thus in line with past investigations that did not show gender-related differences on the various forms of motivation (e.g., Hui et al., 2011), suggesting that profile structure may be similar across gender groups (e.g., Ullrich-French et al., 2016), or revealing a lack of relation between the likelihood of profile membership and these demographic characteristics (e.g., Gillet, Berjot et al., 2012; Gillet et al., 2009).

\section{The Association between of Students' Motivation Profiles and Vitality}

Another key objective of this study was to document the implications of students' motivational profiles in terms of well-being, as indicated by their general level of vitality, a well-known driver of 
positive educational outcomes (e.g., Coffey et al., 2016). In response to our third research question, our results showed that the motivation profiles presented a generally well-differentiated pattern of associations with vitality that generalized across age and gender. Our results also partially supported Hypotheses 4 and 5 showing that profiles characterized by high levels of autonomous motivation and low levels of amotivation (Multifaceted and Knowledge-Oriented) presented higher vitality than profiles characterized by the opposite pattern (Unmotivated). Students from the Controlled profile also showed a similarly low level of vitality. However, contrary to our expectations, the highest levels of vitality was observed in the most unexpected profile, the Hedonist one.

These results mostly support SDT expectations (Ryan \& Deci, 2017) and results from previous research (e.g., Guay et al., 2008) regarding the desirability of autonomous forms of motivation, and the generally undesirable effects associated with controlled forms of motivation or amotivation. In particular, our results support emerging research evidence suggesting that the combination of low levels of autonomous motivation with high levels of controlled motivation (i.e. the Controlled profile) appeared to be associated with the most undesirable covariates levels, rather than the combined presence of autonomous and controlled motivation (i.e., Multifaceted) (Liu et al., 2009; Ratelle et al., 2007).

Unexpectedly, the highest level of vitality, which was assessed generally and not specifically in the context of education, was higher in the Hedonist profile, which presented the highest level of amotivation. However, the very high levels of intrinsic motivation to stimulation and the moderately high level of identified regulation also observed in this profile could have help to counter the negative effect of academic amotivation on general well-being. In contrast, students from the Unmotivated and Controlled profiles presented the lowest levels of vitality. Intrinsic motivation to experience stimulation may be similar to passion, and could thus bring energy to Hedonist students who might already be more strongly oriented toward pleasure and less oriented toward more formal academic activities. These interpretations remain speculative and further research including a broader range of academic and non-academic covariates is needed to better grasp the nature of this Hedonist profile and its true desirability. Thus, although these students show high levels of vitality, what remains to be seen is how this apparent advantage would translate to academic covariates such as achievement, graduation, etc. For instance, Hedonist students might particularly be at risk of dropping out or changing program due to their high level of amotivation.

Finally, our results provide support for the tripartite model of intrinsic motivation (Carbonneau et al., 2012) suggesting that intrinsic motivation to know, to accomplish, and to experience stimulation really reflect three distinct forms of intrinsic, associated with well-differentiated covariates (Hein, Müür, \& Koka, 2004). Although the present results suggest that the most desirable covariates levels might be associated with intrinsic motivation to experience stimulation, the present study focused on a single specific covariate so that the relative desirability of each form of intrinsic motivation for a greater variety of covariates (e.g., achievement, performance anxiety, dropout) should be further investigated in the future. Reinforcing this cautionary note, Carbonneau et al. (2012) showed that intrinsic motivation to experience stimulation tended to present specific relations with affective states related to stimulation (e.g., excited, entertained). More generally, research conducted in work (Van den Broeck, Lens, De Witte, \& Van Coillie, 2013) and educational (Ratelle et al., 2007) contexts clearly demonstrated that the effects of motivational profiles differed as a function of the covariate under study.

\section{The Role of Parents in the Prediction of Students' Motivation Profiles}

Our final objective was to examine the role of parental involvement, warmth, and autonomy support in the prediction of profile membership. Few studies have investigated the determinants of motivation profiles in education (Vansteeenkiste et al., 2009) and, to the best of our knowledge, no study has considered the role of these parental behaviors in motivation profiles. The present results first showed that higher levels of perceived parental warmth received from both the mother and the father predicted an increased likelihood of membership into profiles characterized by high levels of intrinsic motivation to know and low levels of amotivation (i.e., the Knowledge-Oriented and Multifaceted profiles). This result is partly in line with Hypothesis 6 and with past studies showing that warmth fosters autonomous forms of motivation (Hughes et al., 2012; Pomerantz et al., 2012). Still, this profiles was also characterized by high levels of controlled motivation, which did not match Hypothesis 6. Yet, through its association with the development of a stronger bond with their parents, it is plausible for warmth to generate for some students a stronger desire to succeed in order to avoid disappointing their parents. Further research assessing parental values could also bring light to this result. 
The role of parental autonomy support received the most scrutiny among parental behaviors in the self-determination literature and robust evidence exists on its benefits for students' motivational functioning (see Pomerantz \& Grolnick, 2009; Pomerantz et al., 2012). While past studies adopted a variable-centered approach and focused on younger students, there are indications that parental autonomy support is also important in emerging adulthood (e.g., Ratelle, Simard, \& Guay, 2013). However, the present results failed to support Hypotheses 6 and 7, rather showing a limited contribution of autonomy support. Maternal autonomy support did not predict membership in the motivational profiles, and paternal autonomy support only increased the likelihood of membership in the Multifaceted profile in comparison to the Hedonist one, but not into any of the other profiles. This last result still partly matches those from previous studies (Grolnick et al., 1997; McDavid, Cox, \& Amorose, 2012) regarding students' motivational benefits of parental autonomy support for students' intrinsic motivation to know and to accomplish. However, it is important to keep in mind that levels of introjected and external regulations were also high in the Multifaceted profile, which provides a slightly distinct perspective than that stemming from previous research. The very distinct level of amotivation which characterized the Hedonist (very high) and the Multifaceted profile (low) could also explain this difference. This limited but positive contribution of parental autonomy support is also in line with previous research showing that fatheroffspring interactions are described as more oriented toward achievement and mastery than motheroffspring interactions, characterized by stronger closeness and disclosure (Collins \& Russell, 1991).

Contrary to Hypothesis 7, paternal involvement was associated with a greater likelihood of membership into the Unmotivated profile relative to the Knowledge-Oriented and Controlled profiles. In the interpretation of this result, it is important to keep in mind that this effect comes from a multivariate analysis in which other paternal behaviors are taken into account, and thus reflects the effects of paternal involvement net of what this involvement shares with paternal warmth and support. Keeping also in mind that the current sample comprises university students and that a key developmental task of the postsecondary school transition into adulthood is to gain autonomy from parents, even though they remain an important source of support (Scabini, Marta, \& Lanz, 2006), the current result suggests that levels of involvement from the father into the lives of university students that are not balanced by matching levels of warmth and support may contribute to limit autonomous and controlled motivations and increase amotivation. This negative contribution might reflect the fact that, for university students, having their father demonstrate high levels of involvement might be seen as intrusive and infantilizing, calling into question their feelings of competencies. It is also plausible that fathers increase their involvement when students experience motivational difficulties, which could not be tested adequately with the present research design. Future research would therefore benefit from using a longitudinal design that would allow testing cross-lagged relationships between students' motivational profiles and paternal involvement.

Finally, in regard to our Research Question 4, these findings have important implications for the differential role mothers and fathers play in students' motivational functioning. As mentioned above, the nature of mother-student and father- student relationships tends to be different (Collins \& Russell, 1991). What our findings further suggest is that, in the context of university studies, mothers' contribution operates through the warmth component while fathers' contribution occurs through autonomy support, involvement, and warmth. Indeed, once the effect of maternal warmth was taken into account, no other maternal behavior was found to significantly predict profile membership, suggesting that maternal warmth represents a particularly key parental behavior for university students' motivation. In contrast, all studied paternal behaviors contributed to students' motivational profiles, clearly supporting the importance of pursuing this line of inquiry into the relative contribution of mothers and fathers to students' academic motivation, even at the university level. Unfortunately, researchers often combine maternal and paternal behaviors into a single measure of parental behaviors or directly ask students to focus on their parents in an undifferentiated manner (e.g., Gagné, Ryan, \& Bargmann, 2003). The few exceptions to these approaches (e.g., Guay, Ratelle, Larose, Vallerand, \& Vitaro, 2013; McDavid et al., 2012) suggest that treating mothers and fathers separately may provide a more nuanced understanding of parental influence. It is also interesting to note that this maternal and paternal distinctions and relations with profile membership remained stable across age and gender groups (Research Question 5). While it was not possible to consider maternal and paternal behaviors in a single model in the current study due to nonconvergence, it is a worthwhile direction for future research.

\section{Limitations and Directions for Future Research}

When interpreting the present findings, it is important to keep in mind the limits of the study. 
First, we used self-report measures and such measures can be impacted by social desirability and selfreport biases. We thus encourage researchers to conduct additional research using objective (e.g., achievement, dropout) and informant-reported (e.g., teacher reports of learning strategies, engagement, and creativity) measures. Second, our study is based on a cross-sectional design, making it impossible to reach clear conclusions regarding the directionality of the observed associations among constructs. As such, future investigations would benefit from longitudinal studies allowing for a more precise investigation of the stability of profiles over time, and of the direction of the associations between the profiles, their predictors, and vitality. Third, we only considered a limited set of predictors (parental involvement, autonomy support, and warmth as predictors) and one covariate (vitality). Future research is needed to consider a more diversified set of predictors and covariates of students' motivation profiles. For instance, in line with recent research (Michou, Matos, Gargurevich, Gumus, \& Herrera, 2016; van der Kaap-Deeder et al., 2016) showing that motive dispositions (Lang \& Fries, 2006) are linked to autonomous and controlled forms of motivation, these future studies might assess dimensions such as motive to succeed, motives to avoid failure, and contingent self-esteem. Although vitality has been suggested as a relevant indicator of well-being which presents strong relations with academic motivation among undergraduate students (Miquelon, Vallerand, Grouzet, \& Cardinal, 2005), a more complete understanding of the meaning and implications of the various profiles would require the consideration of a more diversified set of covariates covering both academic and non-academic lives of participants. Given the wide range of non-academic variables which may serve to influence students' global levels of vitality (e.g., perceived needs' support from the romantic partner; Patrick, Knee, Canevello \& Lonsbary, 2007) in addition to their academic motivation profiles, the observed associations might have been more pronounced had we considered academic vitality levels.

Fourth, academic motivation was solely assessed with the AMS, in order to build up on previous studies of academic motivation profiles (see Baars \& Wijnia, 2018 for a review) and to measure the three types of intrinsic motivations. Future research should look at the generalizability of the obtained profiles (e.g., the presence of the Hedonist and the absence of a purely autonomous profile [HAu-LC-Lam] ) using other measures of academic motivation in a SDT perspective. Finally, the motivation profiles reported in the present study were observed only in undergraduate students taking an introductory class on organizational behavior in a Canadian university. No information was available regarding students' program, ethnicity, or the number of completed semesters at university, which limits the generalizability of our results. In addition, although the categorization of participants into three distinct age groups helped us to systematically test the similarity of the profiles as a function of age, this categorization remains artificial and could have been strengthened using more precise information on students' academic background. The transposition of the profile similarity framework adopted in the present study should thus be seen as an important area of future development for future statistical research. Future research should also examine whether the same profiles emerge in student samples attending different academic levels (e.g., primary, secondary, graduate), from different countries or cultural backgrounds (e.g., Chan et al., 2015). In particular, the question of how amotivation may affect students' functioning when it is, or not, combined with matching levels of intrinsic motivation to experience stimulation clearly warrants additional studies, both within and across cultures.

\section{Practical Implications}

From a practical perspective, our results suggest that teachers and parents should be particularly attentive to students displaying a profile mainly characterized by controlled motivation (i.e., the Controlled profile) as well as those with globally low levels of motivation (i.e., the Unmotivated profile) as we found these individuals to be at risk for low vitality. In the self-determination literature, numerous studies demonstrated that teachers' autonomy-supportive behaviors were positively related to autonomous motivation (e.g., Hagger, Sultan, Hardcastle, \& Chatzisarantis, 2015; Leptokaridou, Vlachopoulos, \& Papaioannou, 2016; see Ryan \& Deci, 2017). While the contribution of teachers is an important variable to consider when examining students' motivational profiles, the context of university studies implies that students are typically less exposed to each professor. The contribution of parents can therefore be more stable and enduring, and consequently more informative in the prediction of university students' motivational profiles. Along this line, an important conclusion from the present study is related to the importance of warmth from both parents in the emergence of more autonomously-motivated profiles. Although other types of maternal behaviors do not seem to impact students' motivation, paternal involvement into the lives of these developing adults could be particularly problematic, especially when 
not balanced by warmth and support. These results appear to support the value of educative parent-focused interventions centered on a presentation of the key developmental need for autonomy of emerging adults and how these differ from those of children and adolescents. Interventions from university authorities (e.g., the office of student services) should therefore target their students' parent and inform them of their importance in supporting their offspring's motivational functioning and ensuing well-being through acceptance and warmth in a way that supports them without thwarting their feelings of competence. Whereas warmth appears to help in nurturing this budding autonomy, over involvement could rather be seen as intrusive or worse, as curbing students' motivation for their chosen field of study.

\section{References}

Arnett, J. J. (2000). Emerging adulthood: A theory of development from the late teens through the twenties. American Psychologist, 55, 469-480.

Asparouhov, T., \& Muthén, B.O. (2014). Auxiliary variables in mixture modeling: Three-step approaches using Mplus. Structural Equation Modeling, 21, 1-13.

Baars, M., \& Wijnia, L. (2018). The relation between task-specific motivational profiles and training of self-regulated learning skills. Learning and Individual Differences, 64, 125-137.

Bauer, D.J., \& Curran, P.J. (2004). The integration of continuous and discrete latent variable models: Problems and promising opportunities. Psychological Methods, 9, 3-29.

Boiché, J., Sarrazin, P.G., Grouzet, F.M.E., Pelletier, L.G., \& Chanal, J. (2008). Students' motivational profiles and achievement outcomes in physical education: A self-determination perspective. Journal of Educational Psychology, 10, 688-701.

Boiché, J., \& Stephan, Y. (2014). Motivational profiles and achievement: A prospective study testing potential mediators. Motivation and Emotion, 38, 79-92.

Bonneville-Roussy, A., Evans, P., Verner-Filion, J., Vallerand, R.J., \& Bouffard, T. (2017). Motivation and coping with the stress of assessment: Gender differences in outcomes for university students. Contemporary Educational Psychology, 48, 28-42.

Burton, K.D., Lydon, J.E., D'Alessandro, D.U., \& Koestner, R. (2006). The differential effects of intrinsic and identified motivation on well-being and performance. Journal of Personality \& Social Psychology, 91, 750-762.

Cannard, C., Lannegrand-Willems, L., Safont-Mottay, C., \& Zimmermann, G. (2016). Academic amotivation in light of the dark side of identity formation. Journal of Adolescence, 47, 179-184

Carbonneau, N., Vallerand, R.J., \& Lafrenière, M.-A.K. (2012). Toward a tripartite model of intrinsic motivation. Journal of Personality, 80, 1147-1178.

Chan, D.K.C., Yang, S.X., Hamamura, T., Sultan, S., Xing, S., Chatzisarantis, N.L.D., \& Hagger, M.S. (2015). In-lecture learning motivation predicts students' motivation, intention, and behaviour for after-lecture learning: Examining the trans-contextual model across universities from UK, China, and Pakistan. Motivation and Emotion, 39, 908-925.

Coffey, J.K., Wray-Lake, L., Mashek, D., \& Branand, B. (2016). A multi-study examination of well-being theory in college and community samples. Journal of Happiness Studies, 17, 187-211.

Collins, W., \& Russell, G. (1991). Mother-child and father-child relationships in middle adolescence: A developmental analysis. Developmental Review, 11,99-136.

Corpus, J.H., McClintic-Gilbert, M.S., \& Hayenga, A.O. (2009). Within-year changes in children's intrinsic and extrinsic motivational orientations: Contextual predictors and academic outcomes. Contemporary Educational Psychology, 34, 154-166.

Cox, A.E., Ullrich-French, S., \& Sabiston, C. (2013). Using motivation regulations in a person-centered approach to examine the link between social physique anxiety in physical education and physical activity-related outcomes in adolescents. Psychology of Sport \& Exercise, 14, 461-467.

Deci, E.L., \& Ryan, R.M. (2000). The "what" and "why" of goal pursuits: Human needs and the selfdetermination of behavior. Psychological Inquiry, 11, 227-268.

Diallo, T.M.O., \& Lu, H. (2017). On the application of the three-step approach to growth mixture models. Structural Equation Modeling, 24, 714-732.

Diallo, T.M.O, Morin, A.J.S. \& Lu, H. (2016). Impact of misspecifications of the latent variancecovariance and residual matrices on the class enumeration accuracy of growth mixture models. Structural Equation Modeling, 23, 507-531.

Diallo, T.M.O, Morin, A.J.S. \& Lu, H. (2017). The impact of total and partial inclusion or exclusion of active and inactive time invariant covariates in growth mixture models. Psychological Methods, 22, 
166-190.

Enders, C. (2010). Applied missing data analysis. New York, NY: Guilford.

Fousiani, K., Van Petegem, S., Soenens, B., Vansteenkiste, M., \& Chen, B. (2014). Does parental autonomy support relate to adolescent autonomy? An in-depth examination of a seemingly simple question. Journal of Adolescent Research, 29, 299-330.

Gagné, M., Ryan, R.M., \& Bargmann, K. (2003). Autonomy support and need satisfaction in the motivation and well-being of gymnasts. Journal of Applied Sport Psychology, 15, 372-390.

Gillet, N., Becker, C., Lafrenière, M.-A.K.., Huart, I., \& Fouquereau, E. (2017). Organizational support, job resources, soldiers' motivational profiles, work engagement, and affect. Military Psychology, 29, 418-433.

Gillet, N., Berjot, S., Vallerand, R.J., Amoura, S., \& Rosnet, E. (2012). Examining the motivationperformance relationship in competitive sport: A cluster-analytic approach. International Journal of Sport Psychology, 43, 79-102.

Gillet, N., Fouquereau, E., Vallerand, R.J., Abraham, J., \& Colombat, P. (2017). The role of workers' motivational profiles in affective and organizational factors. Journal of Happiness Studies. Advance online publication. doi: 10.1007/s10902-017-9867-9

Gillet, N., Huyghebaert, T., Barrault, S., Bucourt, E., Gimenes, G., Maillot, A., . . Sorel, O. (2017). Autonomous and controlled reasons underlying self-approach and self-avoidance goals and educational outcomes. Social Psychology of Education, 20, 179-193.

Gillet, N., Vallerand, R.J., \& Lafrenière, M. (2012). Intrinsic and extrinsic school motivation as a function of age: Mediating role of autonomy support. Social Psychology of Education, 15, 77-95.

Gillet, N., Vallerand, R.J., \& Paty, B. (2013). Situational motivational profiles and performance with elite performers. Journal of Applied Social Psychology, 43, 1200-1210.

Gillet, N., Vallerand, R.J., \& Rosnet, E. (2009). Motivational clusters and performance in a real-life context. Motivation and Emotion, 33, 49-62.

Gnambs, T., \& Hanfstingl, B. (2016). The decline of academic motivation during adolescence: An accelerated longitudinal cohort analysis on the effect of psychological need satisfaction. Educational Psychology, 36, 1691-1705.

González, A., Paoloni, V., Donolo, D., \& Rinaudo, C. (2012). Motivational and emotional profiles in university undergraduates: A self-determination theory perspective. The Spanish Journal of Psychology, 15, 1069-1080.

Gottfried, A.E., Marcoulides, G.A., Gottfried, A.W., Oliver, P., \& Guerin, D. (2007). Multivariate latent change modeling of developmental decline in academic intrinsic math motivation and achievement: Childhood through adolescence. International Journal of Behavioral Development, 31, 317-327.

Graham, J.W. (2012). Missing data: Analysis and design. New York, NY: Springer.

Grolnick, W. S. (2003). The psychology of parental control: How well-meant parenting backfires. Mahwah, NJ: Erlbaum.

Grolnick, W.S., Deci, E.L., \& Ryan, R.M. (1997) Internalization within the family: The self-determination theory perspective. In J.E. Grusec \& L. Kuczynski (Eds.), Parenting and children's internalization of values (pp. 135-161). New York, NY: Wiley.

Guay, F., Morin, A.J.S., Litalien, D., Valois, P., \& Vallerand, R.J. (2015). Application of exploratory structural equation modeling to evaluate the Academic Motivation Scale. Journal of Experimental Education, 83, 51-82.

Guay, F., Ratelle, C.F., \& Chanal, J. (2008). Optimal learning in optimal contexts: The role of selfdetermination in education. Canadian Psychology, 49, 233-240.

Guay, F., Ratelle, C., Larose, S., Vallerand, R.J., \& Vitaro, F. (2013). The number of autonomy-supportive relationships: Are more relationships better for motivation, perceived competence, and achievement? Contemporary Educational Psychology, 38, 375-382.

Hagger, M.S., Sultan, S., Hardcastle, S.J., \& Chatzisarantis, N.L.D. (2015). Perceived autonomy support and autonomous motivation toward mathematics activities in educational and out-of-school contexts is related to mathematics homework behavior and attainment. Contemporary Educational Psychology, 41, 111-123.

Hayenga, A.O., \& Corpus, J. (2010). Profiles of intrinsic and extrinsic motivations: A person-centered approach to motivation and achievement in middle school. Motivation and Emotion, 34, 371-383.

Hein, V., Müür, M., \& Koka, A. (2004) Intention to be physically active after school graduation and its 
relationship to intrinsic motivation. European Physical Education Review, 10, 5-19.

Hipp, J.R., \& Bauer, D.J. (2006). Local solutions in the estimation of growth mixture models. Psychological Methods, 11, 36-53.

Howard, J. L., Gagné, M., \& Bureau, J. S. (2017). Testing a continuum structure of self-determined motivation: A meta-analysis. Psychological Bulletin. Advance online publication. doi: 10.1037/bul0000125

Howard, J., Gagné, M., Morin, A.J.S., \& Forest, J. (2017). Using bifactor exploratory structural equation modeling to test for a continuum structure of motivation. Journal of Management. Advance online publication. doi: 10.1177/0149206316645653

Hughes, J.N., Wu, J.Y., Kwok, O.M., Villarreal, V., \& Johnson, A. (2012). Indirect effects of child reports of teacher-student relationship on achievement. Journal of Educational Psychology, 104, 350-365.

Hui, E.K.P., Sun, R.C.F., Chow, S.S.-Y., \& Chu, M.H.-T. (2011). Explaining Chinese students' academic motivation: Filial piety and self-determination. Educational Psychology, 31, 377-392.

Lang, J.W.B., \& Fries, S. (2006). A revised 10-item version of the Achievement Motives Scale: Psychometric properties in German-speaking samples. European Journal of Psychological Assessment, 22, 216-224.

Lepper, M.R., Corpus, J.H., \& Iyengar, S.S. (2005). Intrinsic and extrinsic motivational orientations in the classroom. Journal of Educational Psychology, 97, 184-196.

Leptokaridou, E.T., Vlachopoulos, S.P., \& Papaioannou, A.G. (2016). Experimental longitudinal test of the influence of autonomy-supportive teaching on motivation for participation in elementary school physical education. Educational Psychology, 36, 1135-1156.

Litalien, D., Guay, F., \& Morin, A. J. S. (2015). Motivation for PhD studies: Scale development and validation. Learning and Individual Differences, 41, 1-13.

Litalien, D., Morin, A.J.S., Gagné, M., Vallerand, R.J., Losier, G., \& Ryan, R.M. (2017). Evidence of a continuum structure of academic self-determination: A two-study test using a Bifactor-ESEM representation of academic motivation. Contemporary Educational Psychology, 51, 67-82.

Liu, W., Wang, J., Tan, O., Koh, C., \& Ee, J. (2009). A self-determination approach to understanding students' motivation in project work. Learning and Individual Differences, 19, 139-145.

Lo, Y., Mendell, N., \& Rubin, D. (2001). Testing the number of components in a normal mixture. Biometrika, 88, 767-778.

Marsh, H.W., Lüdtke, O., Trautwein, U., \& Morin, A.J.S. (2009). Classical latent profile analysis of academic self-concept dimensions: Synergy of person- and variable-centered approaches to models of self-concept. Structural Equation Modeling, 16, 191-225.

Martinek, D., Hofmann, F., \& Kipman, U. (2016). Academic self-regulation as a function of age: The mediating role of autonomy support and differentiation in school. Social Psychology of Education, 19, 729-748.

McDavid, L., Cox, A.E., \& Amorose, A.J. (2012). The relative roles of physical education teachers and parents in adolescents' leisure-time physical activity motivation and behavior. Psychology of Sport and Exercise, 13, 99-107.

McDonald, R. (1970). Theoretical foundations of principal factor analysis, canonical factor analysis, and alpha factor analysis. British Journal of Mathematical \& Statistical Psychology, 23, 1-21.

McLachlan, G., \& Peel, D. (2000). Finite mixture models. New York, NY: Wiley.

McLarnon, M. J. W., \& O'Neill, T. A. (2018). Extensions of auxiliary variable approaches for the investigation of mediation, moderation, and conditional effects in mixture models. Organizational Research Methods. Advance online publication. doi: 10.1177/1094428118770731

Meyer, J.P., \& Morin, A.J.S. (2016). A person-centered approach to commitment research: Theory, research, and methodology. Journal of Organizational Behavior, 37, 584-612.

Michou, A., Matos, L., Gargurevich, R., Gumus, B., \& Herrera, D. (2016). Building on the enriched hierarchical model of achievement motivation: Autonomous and controlling reasons underlying mastery goals. Psychologica Belgica, 56, 269-287.

Miquelon, P., Vallerand, R. J., Grouzet, F. M. E., \& Cardinal, G. (2005). Perfectionism, academic motivation, and psychological adjustment: An integrative model. Personality and Social Psychology Bulletin, 31, 913-921.

Morin, A.J.S. (2016). Person-centered research strategies in commitment research. In J.P. Meyer (Ed.), The handbook of employee commitment (pp. 490-508). Cheltenham, UK: Edward Elgar. 
Morin, A.J.S., \& Litalien, D. (2017). Webnote: Longitudinal tests of profile similarity and latent transition analyses. Montreal, QC: Substantive Methodological Synergy Research Lab.

https://smslabstats.weebly.com/webnotes.html smslabstats.weebly.com/uploads/1/0/0/6/100647486/lta_distributional_similarity_v02.pdf

Morin, A.J.S., Maïano, C., Nagengast, B., Marsh, H.W., Morizot, J., \& Janosz, M. (2011). Growth mixture modeling of adolescents trajectories of anxiety: The impact of untested invariance assumptions on substantive interpretations. Structural Equation Modeling, 18, 613-648.

Morin, A.J.S., \& Marsh, H.W. (2015). Disentangling shape from levels effects in person-centred analyses: An illustration based on University teacher multidimensional profiles of effectiveness. Structural Equation Modeling, 22, 39-59.

Morin, A.J.S., Meyer, J.P., Creusier, J., \& Biétry, F. (2016). Multiple-group analysis of similarity in latent profile solutions. Organizational Research Methods, 19, 231-254.

Morin, A.J.S., Morizot, J., Boudrias, J.-S., \& Madore, I. (2011). A multifoci person-centered perspective on workplace affective commitment: A latent profile/factor mixture analysis. Organizational Research Methods, 14, 58-90.

Morin, A.J.S., \& Wang, J.C.K. (2016). A gentle introduction to mixture modeling using physical fitness data. In N. Ntoumanis \& N. Myers (Eds.), An introduction to intermediate and advanced statistical analyses for sport and exercise scientists (pp. 183-210). London, UK: Wiley.

Mouratidis, A., \& Michou, A. (2011). Self-determined motivation and social achievement goals in children's emotions. Educational Psychology, 31, 67-86.

Mouratidis, A., Vansteenkiste, M., Lens, W., \& Sideridis, G. (2008). The motivating role of positive feedback in sport and physical education: Evidence for a motivational model. Journal of Sport \& Exercise Psychology, 30, 240-268.

Muthén, L.K., \& Muthén, B.O. (2014). Mplus user guide. Los Angeles, CA: Muthén \& Muthén.

Nishimura, T., \& Sakurai, S. (2017). Longitudinal changes in academic motivation in Japan: Selfdetermination theory and East Asia. Journal of Applied Developmental Psychology, 48, 42-48.

Nylund, K.L., Asparouhov, T., \& Muthén, B. (2007). Deciding on the number of classes in latent class analysis and growth mixture modeling. A Monte Carlo simulation study. Structural Equation Modeling, 14, 535-569.

Patrick, H., Knee, C. R., Canevello, A., \& Lonsbary, C. (2007). The role of need fulfillment in relationship functioning and well-being: A self-determination theory perspective. Journal of Personality and Social Psychology, 92(3), 434-457. http://doi.org/10.1037/0022-3514.92.3.434

Peugh, J., \& Fan, X. (2013). Modeling unobserved heterogeneity using latent profile analysis: A Monte Carlo simulation. Structural Equation Modeling, 20, 616-639.

Pomerantz, E.M., \& Grolnick, W. S. (2009). Toward a clear and inclusive conceptualization of parental control: Reply to the commentaries. Child Development Perspectives, 3, 176-177.

Pomerantz, E. M., Kim, E. M., \& Cheung, C. S. (2012). Parents' involvement in children's learning. In K. R. Harris, S. Graham, T. C. Urdan, S. Graham, J. M. Royer, and M. Zeidner (Eds.), APA educational psychology handbook (pp. 417-440). Washington, DC: American Psychological Association.

Raftery, A. (1995). Bayesian model selection. Sociological Methodology, 25, 111-164.

Ratelle, C.F. \& Duchesne, S. (2017). The role of parents in supporting adaptation in school: A motivational perspective. In H.W. Marsh, R.G. Craven, D.M. McInerney \& F. Guay (Eds), SELF Driving Positive Psychology and Wellbeing International (Advances in Self Research, Vol. 6). Charlotte, NC: Information Age.

Ratelle, C.F., Guay, F., Larose, S., \& Senécal, C. (2004). Family correlates of trajectories of academic motivation during a school transition: A semiparametric group-based approach. Journal of Educational Psychology, 96, 743-754.

Ratelle, C.F., Guay, F., Vallerand, R.J., Larose, S., \& Senécal, C. (2007). Autonomous, controlled, and amotivated types of academic motivation: A person-oriented analysis. Journal of Educational Psychology, 99, 734-746.

Ratelle, C.F., Simard, K., \& Guay, F. (2013). University students' subjective well-being: The role of autonomy support from parents, friends, and romantic partner. Journal of Happiness Studies, 14, 893910.

Raykov, T., \& Marcoulides, G.A. (2004). Using the delta method for approximate interval estimation of parameter functions in SEM. Structural Equation Modeling, 11, 621-637. 
Robbins, R.J. (1994). An assessment of perceptions of parental autonomy support and control: Child and parent correlates. Unpublished Doctoral Dissertation, Department of Psychology, University of Rochester, New York.

Ryan, R.M., \& Deci, E.L. (2017). Self-determination theory: Basic psychological needs in motivation, development, and wellness. New York, NY: Guilford.

Ryan, R.M., \& Frederick, C. (1997). On energy, personality, and health: Subjective vitality as a dynamic reflection of well-being. Journal of Personality, 65, 529-565.

Ryan, R.M., Rigby, S., \& King, K. (1993). Two types of religious internalization and their relation to religious orientations and mental health. Journal of Personality \& Social Psychology, 65, 586-596.

Scabini, E., Marta, E., \& Lanz, M. (2006). Transition to adulthood and family relations: An intergenerational perspective. New York, NY: Psychology Press.

Seghers, J., Vissers, N., Rutten, C., Decroos, S., \& Boen, F. (2014). Intrinsic goals for leisure-time physical activity predict children's daily step counts through autonomous motivation. Psychology of Sport and Exercise, 15, 247-254.

Skrondal, A., \& Laake, P. (2001). Regression among factor scores. Psychometrika, 66, 563-576.

Smith, J.L., Deemer, E.D., Thoman, D.B., \& Zazworsky, L. (2014). Motivation under the microscope: Understanding undergraduate science students' multiple motivations for research. Motivation and Emotion, 38, 496-512.

Solinger, O.N., Van Olffen, W., Roe, R.A., \& Hofmans, J. (2013). On becoming (un)committed: A taxonomy and test of newcomer onboarding scenarios. Organization Science, 24, 1640-1661.

Steinley, D., \& McDonald, R.P. (2007). Examining factor scores distributions to determine the nature of latent spaces. Multivariate Behavioral Research, 42, 133-156.

Tofighi, D., \& Enders, C. (2008). Identifying the correct number of classes in growth mixture models. In G.R. Hancock \& K.M. Samuelsen (Eds.), Advances in latent variable mixture models (pp. 317-341). Charlotte, NC: Information Age.

Ullrich-French, S., \& Cox, A. (2009). Using cluster analysis to examine the combinations of motivation regulations of physical education students. Journal of Sport \& Exercise Psychology, 31, 358-379.

Ullrich-French, S., Cox, A.E., \& Cooper, B.R. (2016). Examining combinations of social physique anxiety and motivation regulations using latent profile analysis. Measurement in Physical Education and Exercise Science, 20, 63-74.

Vallerand, R.J. (1997). Toward a hierarchical model of intrinsic and extrinsic motivation. In M. Zanna (Ed.), Advances in experimental social psychology (pp. 271-360). New York: Academic Press.

Vallerand, R.J., Fortier, M.S., \& Guay, F. (1997). Self-determination and persistence in a real-life setting: Toward a motivational model of high school dropout. Journal of Personality and Social Psychology, 72, 1161-1176.

Vallerand, R.J., Pelletier, L.G., Blais, M.R., Brière, N.M., Senécal, C., \& Vallières, E.F. (1992). The Academic Motivation Scale: A measure of intrinsic, extrinsic, and amotivation in education. Educational and Psychological Measurement, 52, 1003-1017.

Vallerand, R.J., Pelletier, L.G., Blais, M.R., Brière, N.M., Senécal, C., \& Vallières, E.F. (1993). On the assessment of intrinsic, extrinsic, and amotivation in education: Evidence on the concurrent and construct validity of the Academic Motivation Scale. Educational and Psychological Measurement, $53,159-172$.

Van den Broeck, A., Lens, W., De Witte, H., \& Van Coillie, H. (2013). Unraveling the importance of the quantity and the quality of workers' motivation for well-being: A person-centered perspective. Journal of Vocational Behavior, 82, 69-78.

van der Kaap-Deeder, J., Wouters, S., Verschueren, K., Briers, V., Deeren, B., \& Vansteenkiste, M. (2016). The pursuit of self-esteem and motivation. Psychologica Belgica, 56, 143-168.

Vansteenkiste, M., Sierens, E., Soenens, B., Luyckx, K., \& Lens, W. (2009). Motivational profiles from a self-determination perspective: The quality of motivation matters. Journal of Educational Psychology, 101, 671-688.

Wang, J.C.K., Morin, A.J.S., Ryan, R.M., \& Liu, W.C. (2016). Students' motivational profiles in the physical education context. Journal of Sport \& Exercise Psychology, 38, 612-630. 


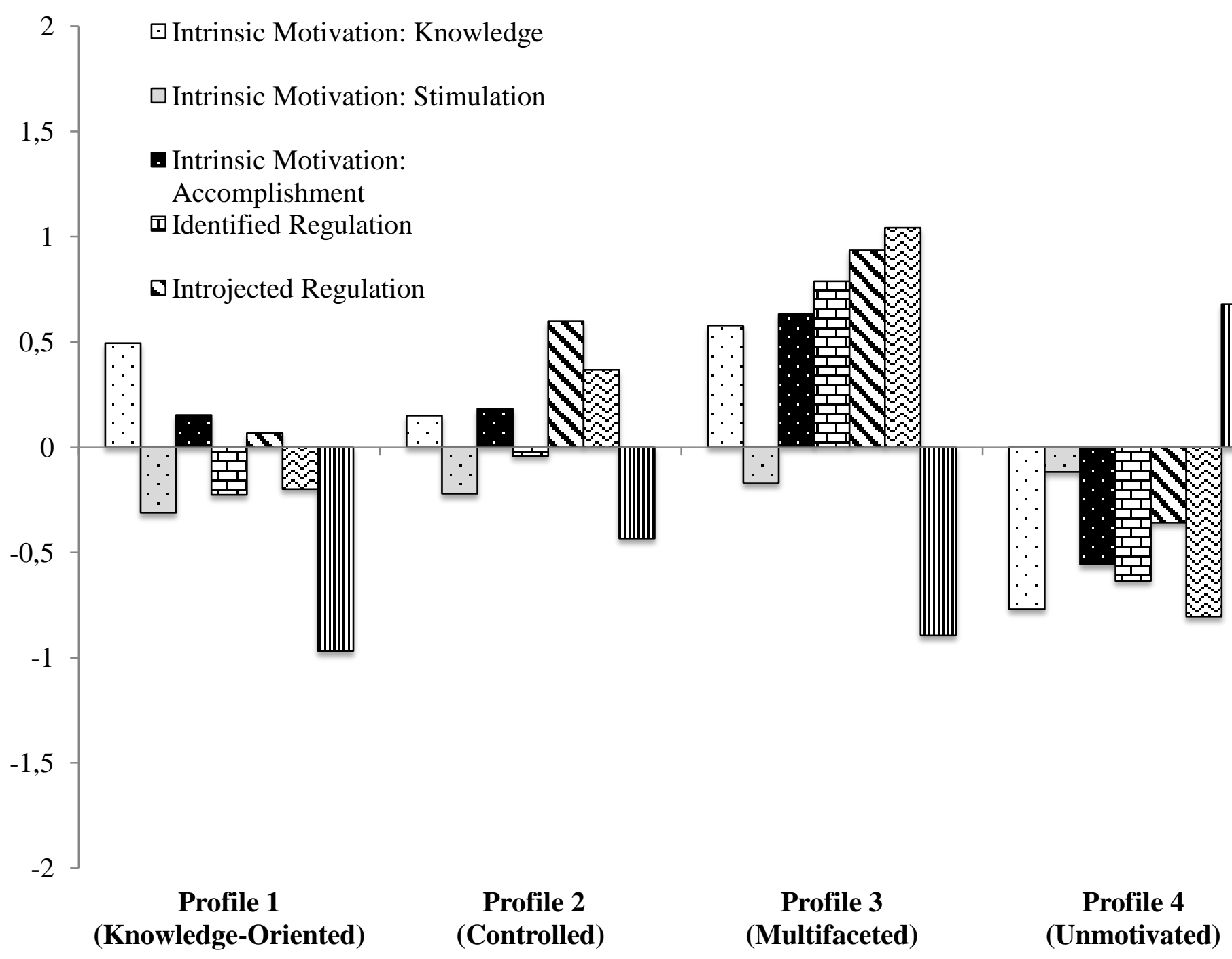

Figure 1. Final Latent Profile Solution. 
Table 1

Correlations Between all Variables Used in the Present Study (Factor Scores)

\begin{tabular}{lllllllllll}
\hline & IMKN & IMSTI & IMACC & IDER & INJR & EXTR & AMOT & VITA & MINV & MAUTS \\
\hline IMKN & .79 & & & & & & & & & \\
IMSTI & $.41^{* *}$ & .87 & & & & & & & & \\
IMACC & $.68^{* *}$ & $.45^{* *}$ & .71 & & & & & & & \\
IDER & $.40^{* *}$ & $.26^{* *}$ & $.27^{* *}$ & .81 & & & & & & \\
INJR & $.37^{* *}$ & $-.22^{* *}$ & $.45^{* *}$ & $.08^{*}$ & .86 & & & & & \\
EXTR & $.38^{* *}$ & $.09^{* *}$ & $.46^{* *}$ & $.49^{* *}$ & $.38^{* *}$ & .83 & & & & \\
AMOT & $-.37^{* *}$ & $.42^{* *}$ & $-.28^{* *}$ & $.06^{*}$ & $-.66^{* *}$ & $-.30^{* *}$ & .95 & & & \\
VITAL & $.25^{* *}$ & .11 & $.13^{*}$ & .09 & .06 & -.01 & -.16 & .86 & & \\
MINV & $.18^{* *}$ & .07 & $.14^{* *}$ & $.16^{* *}$ & .04 & $.13^{*}$ & -.07 & $.31^{* *}$ & .68 & \\
MAUTS & $.15^{* *}$ & $-.14^{*}$ & $.11^{*}$ & .09 & $.23^{* *}$ & .10 & $-.30^{* *}$ & $.26^{* *}$ & $.45^{* *}$ & .84 \\
MWAR & $.19^{* *}$ & $-.28^{* *}$ & $.14^{* *}$ & .03 & $.30^{* *}$ & $.23^{* *}$ & $-.49^{* *}$ & $.23^{* *}$ & $.47^{* *}$ & $.72^{* *}$ \\
FINV & .02 & $.19^{* *}$ & .03 & .11 & -.15 & -.03 & .18 & $.13^{*}$ & $.35^{* *}$ & $.12^{*}$ \\
FAUTS & $.17^{* *}$ & -.08 & .07 & .08 & .10 & .06 & $-.22^{*}$ & $.22^{* *}$ & $.29^{* *}$ & $.38^{* *}$ \\
FWAR & $.17^{* *}$ & -.13 & .10 & .07 & .14 & .08 & $-.27^{* *}$ & $.20^{* *}$ & $.34^{* *}$ & $.42^{* *}$ \\
\hline
\end{tabular}

Note. $* p<.05 ; * * p<.01$; Composite reliability estimates $(\omega)$ are reported in italics in the diagonal; IMKN = intrinsic motivation to know; IMSTI = intrinsic motivation to experience stimulation; IMACC = intrinsic motivation to accomplish; IDER = identified regulation; INJR = introjected regulation; EXTR = external regulation; AMOT $=$ amotivation; VITA $=$ vitality; MINV = mother involvement; MAUTS = mother autonomy support; MWAR = mother warmth; FINV = father involvement; FAUTS $=$ father autonomy support; FWAR = father warmth. 
Table 2

Results from the Latent Profiles Analyses Across Genders and Between Age Groups (5-Profile Solution)

\begin{tabular}{|c|c|c|c|c|c|c|c|c|}
\hline Model & LL & $\begin{array}{l}\# \\
f \\
p\end{array}$ & $\begin{array}{c}\text { Scali } \\
\text { ng }\end{array}$ & AIC & CAIC & $\mathrm{BIC}$ & $\mathrm{ABIC}$ & $\begin{array}{l}\text { Entro } \\
\text { py }\end{array}$ \\
\hline \multicolumn{9}{|l|}{ Gender comparisons } \\
\hline Males: Final 5-Profile Solution & $\begin{array}{l}- \\
3065.4 \\
3\end{array}$ & $\begin{array}{l}8 \\
1\end{array}$ & 1.20 & $\begin{array}{l}6292.8 \\
6\end{array}$ & $\begin{array}{l}6704.8 \\
9\end{array}$ & $\begin{array}{l}6623.8 \\
9\end{array}$ & $\begin{array}{l}6366 . \\
84\end{array}$ & 0.90 \\
\hline Females: Final 5-Profile Solution & $\begin{array}{l}- \\
4039.6 \\
3\end{array}$ & $\begin{array}{l}8 \\
1\end{array}$ & 1.39 & $\begin{array}{l}8241.2 \\
7\end{array}$ & $\begin{array}{l}8680.6 \\
8\end{array}$ & $\begin{array}{l}8599.6 \\
8\end{array}$ & $\begin{array}{l}8342 . \\
52\end{array}$ & 0.88 \\
\hline \multicolumn{9}{|l|}{ Tests of Profile Similarity } \\
\hline Configural Similarity & $\begin{array}{l}- \\
7830.4 \\
3\end{array}$ & $\begin{array}{l}1 \\
5 \\
6\end{array}$ & 1.34 & $\begin{array}{l}15972 . \\
85\end{array}$ & $\begin{array}{l}16903 . \\
11\end{array}$ & $\begin{array}{l}16747 . \\
11\end{array}$ & $\begin{array}{l}16251 \\
.63\end{array}$ & 0.92 \\
\hline Structural Similarity & $\begin{array}{l}- \\
7882.5 \\
9\end{array}$ & $\begin{array}{l}1 \\
2 \\
1\end{array}$ & 1.51 & $\begin{array}{l}16007 . \\
18\end{array}$ & $\begin{array}{l}16728 . \\
73\end{array}$ & $\begin{array}{l}16607 . \\
73\end{array}$ & $\begin{array}{l}16223 \\
.41\end{array}$ & 0.91 \\
\hline Dispersion Similarity & $\begin{array}{l}- \\
7904.9 \\
8\end{array}$ & $\begin{array}{l}8 \\
6\end{array}$ & 1.46 & $\begin{array}{l}15981 . \\
96\end{array}$ & $\begin{array}{l}16494 . \\
79\end{array}$ & $\begin{array}{l}16408 . \\
79\end{array}$ & $\begin{array}{l}16135 \\
.64\end{array}$ & 0.91 \\
\hline Distributional Similarity & $\begin{array}{l}- \\
7916.2 \\
7\end{array}$ & $\begin{array}{l}8 \\
2\end{array}$ & 1.45 & $\begin{array}{l}15996 . \\
54\end{array}$ & $\begin{array}{l}16485 . \\
52\end{array}$ & $\begin{array}{l}16403 . \\
52\end{array}$ & $\begin{array}{l}16143 \\
.07\end{array}$ & 0.91 \\
\hline \multicolumn{9}{|l|}{ Explanatory Similarity } \\
\hline $\begin{array}{l}\text { Relations between profiles and vitality } \\
\text { freely estimated }\end{array}$ & $\begin{array}{l}- \\
3152.4 \\
1\end{array}$ & $\begin{array}{l}1 \\
6\end{array}$ & 0.90 & $\begin{array}{l}6336.8 \\
2\end{array}$ & $\begin{array}{l}6448.2 \\
3\end{array}$ & $\begin{array}{l}6432.2 \\
3\end{array}$ & $\begin{array}{l}6365 . \\
41\end{array}$ & 0.85 \\
\hline $\begin{array}{l}\text { Relations between profiles and vitality } \\
\text { invariant }\end{array}$ & $\begin{array}{l}- \\
3163.3 \\
3\end{array}$ & $\begin{array}{l}1 \\
1\end{array}$ & 0.90 & $\begin{array}{l}6348.6 \\
7\end{array}$ & $\begin{array}{l}6425.2 \\
6\end{array}$ & $\begin{array}{l}6414.2 \\
6\end{array}$ & $\begin{array}{l}6368 . \\
32\end{array}$ & 0.85 \\
\hline \multicolumn{9}{|l|}{ Predictive Similarity (Mothers) } \\
\hline $\begin{array}{l}\text { Relations between predictors and profiles } \\
\text { freely estimated }\end{array}$ & $\begin{array}{l}- \\
4270.7 \\
6\end{array}$ & $\begin{array}{l}4 \\
4\end{array}$ & 1.13 & $\begin{array}{l}8629.5 \\
3\end{array}$ & $\begin{array}{l}8891.9 \\
1\end{array}$ & $\begin{array}{l}8847.9 \\
1\end{array}$ & $\begin{array}{l}8708 . \\
16\end{array}$ & 0.85 \\
\hline $\begin{array}{l}\text { Relations between predictors and profiles } \\
\text { invariant }\end{array}$ & $\begin{array}{l}- \\
4285.6 \\
7\end{array}$ & $\begin{array}{l}3 \\
9\end{array}$ & 1.12 & $\begin{array}{l}8629.3 \\
4\end{array}$ & $\begin{array}{l}8812.2 \\
8\end{array}$ & $\begin{array}{l}8773.2 \\
8\end{array}$ & $\begin{array}{l}8681 . \\
17\end{array}$ & 0.85 \\
\hline \multicolumn{9}{|l|}{ Predictive Similarity (Fathers) } \\
\hline $\begin{array}{l}\text { Relations between predictors and profiles } \\
\text { freely estimated }\end{array}$ & $\begin{array}{l}- \\
4197.7 \\
9\end{array}$ & $\begin{array}{l}4 \\
1\end{array}$ & 1.15 & $\begin{array}{l}8477.5 \\
7\end{array}$ & $\begin{array}{l}8722.0 \\
6\end{array}$ & $\begin{array}{l}8681.0 \\
6\end{array}$ & $\begin{array}{l}8550 . \\
84\end{array}$ & 0.86 \\
\hline $\begin{array}{l}\text { Relations between predictors and profiles } \\
\text { invariant }\end{array}$ & $\begin{array}{l}- \\
4209.2 \\
3\end{array}$ & $\begin{array}{l}2 \\
9\end{array}$ & 1.02 & $\begin{array}{l}8476.4 \\
5\end{array}$ & $\begin{array}{l}8649.3 \\
8\end{array}$ & $\begin{array}{l}8620.3 \\
8\end{array}$ & $\begin{array}{l}8528 . \\
27\end{array}$ & 0.85 \\
\hline \multicolumn{9}{|l|}{ Age group comparisons } \\
\hline 17 to 20: Final 5-Profile Solution & $\begin{array}{l}- \\
2463.1 \\
7\end{array}$ & $\begin{array}{l}8 \\
1\end{array}$ & 1.27 & $\begin{array}{l}5088.3 \\
4\end{array}$ & $\begin{array}{l}5487.2 \\
1\end{array}$ & $\begin{array}{l}5406.2 \\
1\end{array}$ & $\begin{array}{l}5149 . \\
22\end{array}$ & 0.89 \\
\hline 21 to 23: Final 5-Profile Solution & $\begin{array}{l}- \\
2279.7 \\
0\end{array}$ & $\begin{array}{l}8 \\
1\end{array}$ & 1.13 & $\begin{array}{l}4721.4 \\
0\end{array}$ & $\begin{array}{l}5114.4 \\
3\end{array}$ & $\begin{array}{l}5033.4 \\
3\end{array}$ & $\begin{array}{l}4776 . \\
47\end{array}$ & 0.92 \\
\hline 24 or more: Final 5-Profile Solution & $\begin{array}{l}- \\
1618.3 \\
1\end{array}$ & $\begin{array}{l}8 \\
1\end{array}$ & 1.08 & $\begin{array}{l}3398.6 \\
1\end{array}$ & $\begin{array}{l}3761.8 \\
8\end{array}$ & $\begin{array}{l}3680.8 \\
8\end{array}$ & $\begin{array}{l}3424 . \\
13\end{array}$ & 0.90 \\
\hline \multicolumn{9}{|l|}{ Tests of Profile Similarity } \\
\hline Configural Similarity & $\begin{array}{l}- \\
7428.5 \\
0\end{array}$ & $\begin{array}{l}2 \\
3 \\
1\end{array}$ & 1.28 & $\begin{array}{l}15318 . \\
99\end{array}$ & $\begin{array}{l}16674 . \\
97\end{array}$ & $\begin{array}{l}16443 . \\
97\end{array}$ & $\begin{array}{l}15710 \\
.32\end{array}$ & 0.93 \\
\hline Structural Similarity & $\begin{array}{l}- \\
7503.0 \\
4\end{array}$ & $\begin{array}{l}1 \\
6 \\
1\end{array}$ & 1.26 & $\begin{array}{l}15328 . \\
07\end{array}$ & $\begin{array}{l}16273 . \\
15\end{array}$ & $\begin{array}{l}16112 . \\
15\end{array}$ & $\begin{array}{l}15600 \\
.82\end{array}$ & 0.93 \\
\hline Dispersion Similarity & $\begin{array}{l}- \\
7549.7 \\
2\end{array}$ & $\begin{array}{l}9 \\
1\end{array}$ & 1.30 & $\begin{array}{l}15281 . \\
45\end{array}$ & $\begin{array}{l}15815 . \\
62\end{array}$ & $\begin{array}{l}15724 . \\
62\end{array}$ & $\begin{array}{l}15435 \\
.61\end{array}$ & 0.92 \\
\hline Distributional Similarity & 7556.7 & $\begin{array}{l}8 \\
3\end{array}$ & 1.34 & $\begin{array}{l}15279 . \\
57\end{array}$ & $\begin{array}{l}15766 . \\
78\end{array}$ & $\begin{array}{l}15683 . \\
78\end{array}$ & $\begin{array}{l}15420 \\
.18\end{array}$ & 0.92 \\
\hline
\end{tabular}




\section{SUPPLEMENTS FOR ACADEMIC MOTIVATION PROFILES S23}

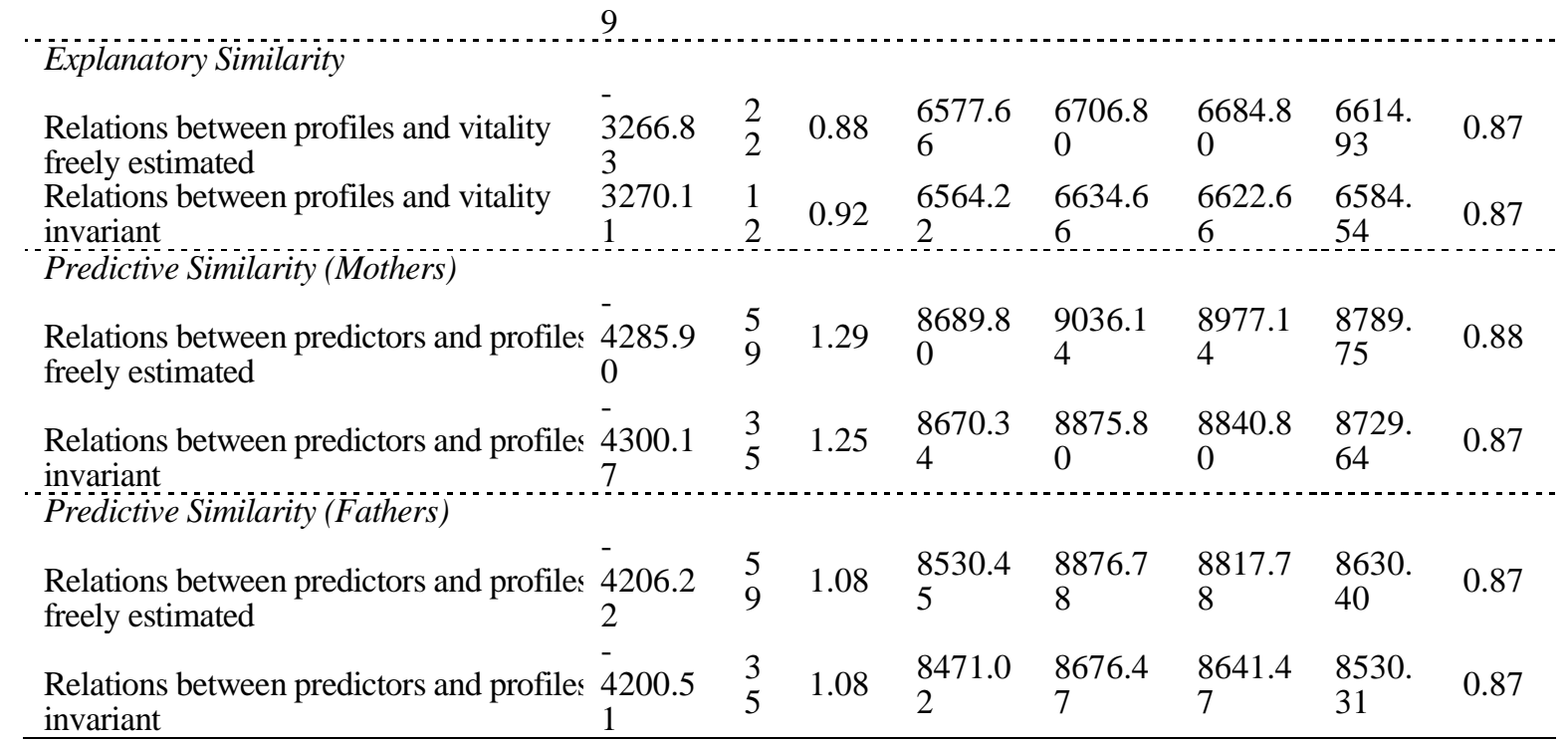

Note. LL: Model LogLikelihood; \#fp: Number of free parameters; Scaling = Scaling correction factor; AIC: Akaike Information Criteria; CAIC: Constant AIC; BIC: Bayesian Information Criteria; ABIC: Sample-Size adjusted BIC; M: Means; V: Variances; P: Class probabilities. 


\section{SUPPLEMENTS FOR ACADEMIC MOTIVATION PROFILES S24}

Table 3

Results from Multinomial Logic Regressions for the Contribution of Perceived Parental Behaviors

\begin{tabular}{|c|c|c|c|c|c|c|c|}
\hline & \multicolumn{2}{|c|}{ Latent profile 1 vs. 2} & \multicolumn{2}{|c|}{ Latent profile 3 vs. 2} & \multicolumn{2}{|c|}{ Latent profile 4 vs. 2} & Latent pr \\
\hline & Coef. (SE) & OR & Coef. (SE) & OR & Coef. (SE) & OR & Coef. (S \\
\hline Mother involvement & $-0.13(0.20)$ & 0.88 & $0.26(0.17)$ & 1.30 & $0.15(0.17)$ & 1.16 & $0.14(0$ \\
\hline Mother autonomy support & $-0.08(0.21)$ & 0.93 & $0.20(0.22)$ & 1.22 & $0.20(0.25)$ & 1.23 & $0.03(0$ \\
\hline Mother warmth & $0.98(0.30)^{* *}$ & 2.67 & $0.15(0.23)$ & 1.16 & $-0.78(0.31)^{*}$ & 0.46 & $-0.54(0$. \\
\hline Father involvement & $-0.24(0.18)$ & 0.79 & $0.22(0.17)$ & 1.25 & $0.65(0.24)^{* *}$ & 1.91 & $0.12(1$ \\
\hline Father autonomy support & $0.17(0.30)$ & 1.18 & $0.26(0.23)$ & 1.29 & $0.05(0.30)$ & 1.05 & $-0.25(0$ \\
\hline \multirow[t]{3}{*}{ Father warmth } & $0.76(0.32)^{*}$ & 2.14 & $0.18(0.27)$ & 1.19 & $-0.57(0.34)$ & 0.57 & $-0.06(0$ \\
\hline & \multicolumn{2}{|c|}{ Latent profile 4 vs. 3} & \multicolumn{2}{|c|}{ Latent profile 5 vs. 3} & \multicolumn{2}{|c|}{ Latent profile 1 vs. 4} & Latent \\
\hline & Coef. (SE) & OR & Coef. (SE) & OR & Coef. (SE) & OR & Coef. (S \\
\hline Mother involvement & $-0.12(0.21)$ & 0.89 & $-0.12(0.31)$ & 0.89 & $-0.27(0.22)$ & 0.76 & $-0.00(0$ \\
\hline Mother autonomy support & $0.01(0.26)$ & 1.01 & $-0.16(0.39)$ & 0.85 & $-0.28(0.27)$ & 0.76 & $-0.17(0$ \\
\hline Mother warmth & $-0.93(0.31)^{* *}$ & 0.40 & $-0.68(0.43)$ & 0.51 & $1.77(0.39)^{* *}$ & 5.84 & $0.25(0$ \\
\hline Father involvement & $0.43(0.25)$ & 1.53 & $-0.10(1.11)$ & 0.91 & $-0.89(0.29) * *$ & 0.41 & $-0.53(1$ \\
\hline Father autonomy support & $-0.21(0.31)$ & 0.81 & $-0.51(0.26)^{*}$ & 0.60 & $0.12(0.35)$ & 1.13 & $-0.30(0$ \\
\hline Father warmth & $-0.75(0.37)^{*}$ & 0.47 & $-0.24(0.50)$ & 0.79 & $1.33(0.41)^{* *}$ & 3.77 & $0.51(0$ \\
\hline
\end{tabular}

Note. ${ }^{*} p<.05 ; * * p<.01$; SE: Standard error of the coefficient; OR: Odds ratio; The coefficients and OR reflects the contribution of the predictors on the likelihood of membership into the first listed profile relative to the second listed profile; Profile 1: Knowledge-Oriented; Profile 2: Controlled; Profile 3: Multifaceted; Profile 4: Unmotivated; Profile 5: Hedonist. 


\section{Online Supplemental Materials for:}

\section{Self-Determined Motivation Profiles among Undergraduate Students: A Robust Test of Profile Similarity as a Function of Gender and Age \\ Authors' note:}

These online technical appendices are to be posted on the journal website and hot-linked to the manuscript. If the journal does not offer this possibility, these materials can alternatively be posted on one of our personal websites (we will adjust the in-text reference upon acceptance).

We would also be happy to have some of these materials brought back into the main manuscript, or included as published appendices if you deem it useful. We developed these materials to provide additional technical information and to keep the main manuscript from becoming needlessly long.

\section{Sections}

1. Preliminary Measurement Models and Tests of Measurement Invariance across Gender and Age Groups.

2. Table S1. Goodness-of-Fit Statistics of the Measurement Models across Gender

3. Table S2. Goodness-of-Fit Statistics of the Measurement Models across Age

4. Table S3. Standardized Parameter Estimates from the Final Measurement Models

5. Table S4. Goodness-of-Fit Results from the Latent Profile Analyses across Gender

6. Table S5. Goodness-of-Fit Results from the Latent Profile Analyses across Age Group

7. Table S6. Detailed Results from the Final LPA Solution of Distributional Similarity

8. Table S7 Means on the Oucome (Vitality) for each Profile and Mean Differences between Profiles

9. Figure S1. Elbow Plot of the Information Criteria for the Latent Profile Analyses (Males).

10. Figure S2. Elbow Plot of the Information Criteria for the Latent Profile Analyses (Females).

11. Figure S3. Elbow Plot of the Information Criteria for the Latent Profile Analyses (17 to 20 years old).

12. Figure S4. Elbow Plot of the Information Criteria for the Latent Profile Analyses (21 to 23 years old).

13. Figure S5. Elbow Plot of the Information Criteria for the Latent Profile Analyses (24 years old or more).

14. Annotated Mplus Codes. 


\section{Preliminary Measurement Models and Tests of Measurement Invariance across Gender and Age Groups}

Preliminary measurement models were estimated using Mplus 7.3 (Muthén \& Muthén, 2014). Due to the complexity of the measurement models underlying all constructs assessed in the present study, these preliminary analyses were conducted separately for the academic motivation scale and the covariates (predictors [perceptions of parents] and covariate [vitality]). These models were estimated as multiple group models, allowing for the estimation of similar models across gender and age groups, and for the progressive integration of invariance constraints to the models. The motivation models included, in each group, seven factors for academic motivation (intrinsic motivation to know, intrinsic motivation to experience stimulation, intrinsic motivation to accomplish, identified regulation, introjected regulation, external regulation, and amotivation). The covariates models included, in each group, six factors related to parental behaviors (perceptions of mother and father involvement, autonomy support, and warmth), and one vitality factor.

Measurement models for academic motivation were estimated using exploratory structural equation modeling (ESEM; Asparouhov \& Muthén, 2009; Marsh et al., 2009; Morin, Marsh, \& Nagengast, 2013). The decision to rely on a ESEM representation of Academic Motivation Scale ratings is based on the results from simulation studies (Asparouhov \& Muthén, 2009; Sass \& Schmitt, 2010; Schmitt \& Sass, 2011) and studies of simulated data (Marsh, Lüdtke, Nagengast, Morin, \& Von Davier, 2013; Morin, Arens, \& Marsh, 2015) showing that forcing cross loadings (even as small as .100) present in the population model to be exactly zero according to typical confirmatory factor analytic (CFA) specification forces these cross loadings to be expressed through the inflation of the factor correlations (for a review, see Asparouhov, Muthén, \& Morin, 2015). In contrast, these same studies showed that the free estimation of cross-loadings, even when none are present in the population model, still provides unbiased estimates of the factor correlations. Furthermore, recent studies have also supported the superiority of an ESEM representation for measures of academic motivation (Guay, Morin, Litalien, Valois, \& Vallerand, 2015; Litalien, Guay, \& Morin, 2015). For similar reasons, ratings of parental behaviors were also represented via an ESEM measurement model, in which one additional CFA factor was integrated to account for ratings of students' levels of vitality.

ESEM factors were specified in a confirmatory manner, using an oblique target rotation (Asparouhov \& Muthén, 2009; Browne, 2001), allowing for the pre-specification of target loadings in a confirmatory manner, while cross-loadings are targeted to be as close to zero as possible, yet still freely estimated. For the covariates model, a priori correlated uniquenesses were also integrated to control for the methodological artefact associated with the parallel wording of a subset of items used to assess maternal and paternal characteristics (e.g., "My mother helps me to choose my own direction" and "My father helps me to choose my own direction"; Marsh, Abduljabbar et al., 2013). One orthogonal method factor was also included to this model to take into account the methodological artefact due to the negative wording of 16 items (e.g., Marsh, Scalas, \& Nagengast, 2010).

All of these measurement models were estimated using the robust maximum Likelihood (MLR) estimator. This estimator provides standard errors and tests of fit that are robust in relation to non-normality and the use of Likert-type rating scales based on seven response categories (Finney \& DiStephano, 2013). Analyses were conducted using the data from all respondents, using Full Information MLR estimation (FIML; Enders, 2010; Graham, 2009) to account for missing data. FIML estimation has been found to result in unbiased parameter estimates under even a very high level of missing data (e.g., 50\%) under Missing At Random (MAR) assumptions, and even in some cases to violations of this assumption (e.g. Enders, 2010; Graham, 2009; Larsen, 2011). 
Before saving the factor scores for our main analyses, we verified that the measurement model operated in the same manner across gender and age groups, through sequential tests of measurement invariance (Millsap, 2011): (1) configural invariance, (2) weak invariance (loadings), (3) strong invariance (loadings and intercepts), (4) strict invariance (loadings, intercepts, and uniquenesses); (5) invariance of the latent variance-covariance matrix (loadings, intercepts, uniquenesses, and latent variances and covariances); and (6) latent means invariance (loadings, intercepts, uniquenesses, latent variances and covariances, and latent means). For the covariates models, an additional step testing the invariance of the parallel-worded correlated uniquenesses was also included.

Given the known oversensitivity of the chi-square test of exact fit $\left(\chi^{2}\right)$ to sample size and minor model misspecifications (e.g., Marsh, Hau, \& Grayson, 2005), we relied on goodnessof-fit indices to describe the fit of the alternative models (Hu \& Bentler, 1999): the comparative fit index (CFI), the Tucker-Lewis index (TLI), as well as the root mean square error of approximation (RMSEA) and its $90 \%$ confidence interval. Values greater than .90 for the CFI and TLI indicate adequate model fit, although values greater than .95 are preferable. Values smaller than .08 or .06 for the RMSEA respectively support acceptable and excellent model fit. Like the chi-square, chi-square difference tests present a known sensitivity to sample size and minor model misspecifications so that recent studies suggest complementing this information with changes in goodness-of-fit indices (Chen, 2007; Cheung \& Rensvold, 2002 ) in the context of tests of measurement invariance. A $\Delta$ CFI/TLI of .010 or less and a $\triangle$ RMSEA of .015 or less between a more restricted model and the preceding one indicate that the invariance hypothesis should not be rejected.

The results from these models are reported in supplementary Tables S1 and S2, for gender and age comparisons, respectively. These results provided clear support to the complete invariance of the models across gender and age groups, with none of the change in goodness-of-fit indices exceeding the recommended guidelines $(\Delta \mathrm{CFI} \leq .010 ; \Delta \mathrm{TLI} \leq .010$; $\triangle \mathrm{RMSEA} \leq .015)$. To ensure that the latent profiles estimated were based on fully comparable measures across gender and age groups, the factor scores used in the main analyses were saved from the most invariant model. Invariant parameter estimates for the motivation and covariates models are reported in Table S3. Although only strict invariance is required to ensure the comparability of factors scores across distinct groups of participants, there are advantages to saving factor scores from a model of complete measurement invariance. More precisely, saving factor scores based on a measurement model in which both the variances and the latent means are equivalent across groups, and respectively constrained to take a value of 1 and 0 in all groups, provides scores on profile indicators that can be readily interpreted in standardized terms as deviation from the grand mean expressed in standard deviation units (Meyer \& Morin, 2016; Morin, 2016).

\section{References used in this supplement}

Asparouhov, T., \& Muthén, B.O. (2009). Exploratory structural equation modeling. Structural Equation Modeling, 16, 397-438.

Asparouhov, T., Muthén, B., \& Morin, A.J.S. (2015). Bayesian structural equation modeling with cross-loadings and residual covariances. Journal of Management, 41, 1561-1577.

Browne, M.W. (2001). An overview of analytic rotation in exploratory factor analysis. Multivariate Behavioral Research, 36, 111-150.

Chen, F.F. (2007). Sensitivity of goodness of fit indexes to lack of measurement. Structural Equation Modeling, 14, 464-504.

Cheung, G.W., \& Rensvold, R.B. (2002). Evaluating goodness-of fit indexes for testing measurement invariance. Structural Equation Modeling, 9, 233-255.

Enders, C. (2010). Applied missing data analysis. New York, NY: Guilford.

Finney, S.J., \& DiStefano, C. (2013). Non-normal and categorical data in structural equation 
modeling. In G.R. Hancock \& R.O. Mueller (Eds), Structural equation modeling: A second course, $2^{\text {nd }}$ edition (pp. 439-492). Charlotte, NC: IAP.

Graham, J.W. (2009). Missing data analysis: Making it work in the real world. Annual Review of Psychology, 60, 549-576.

Guay, F., Morin, A.J.S., Litalien, D., Valois, P., \& Vallerand, R.J. (2015). Application of exploratory structural equation modeling to evaluate the academic motivation scale. Journal of Experimental Education, 83, 51-82.

Hu, L.-T., \& Bentler, P.M. (1999). Cutoff criteria for fit indexes in covariance structure analysis: Conventional criteria versus new alternatives. Structural Equation Modeling, 6, 1-55.

Larsen, R. (2011). Missing data imputation versus full information maximum likelihood with second level dependencies. Structural Equation Modeling, 18, 649-662.

Litalien, D., Guay, F., \& Morin, A.J.S. (2015). Motivation for PhD studies: Scale development and validation. Learning and Individual Differences, 41, 1-13.

Marsh, H.W., Abduljabbar, A.S., Abu-Hilal, M.M., Morin, A.J.S., Abdelfattah, F., Leung, K.C., et al. (2013). Factorial, convergent, and discriminant validity of timss math and science motivation measures: A comparison of Arab and Anglo-Saxon countries. Journal of Educational Psychology, 105, 108-128.

Marsh, H.W., Hau, K., \& Grayson, D. (2005). Goodness of fit in structural equation models. In A. Maydeu-Olivares \& J.J. McArdle (Eds.), Contemporary psychometrics: A festschrift for Roderick P. McDonald. (pp. 275-340). Mahwah, NJ: Erlbaum.

Marsh, H.W., Lüdtke, O., Nagengast, B., Morin, A.J.S., \& Von Davier, M. (2013). Why item parcels are (almost) never appropriate: Two wrongs do not make a right-Camouflaging misspecification with item parcels in CFA models. Psychological Methods, 18, 257284.

Marsh, H.W., Muthén, B., Asparouhov, T., Lüdtke, O., Robitzsch, A., Morin, A.J.S., \& Trautwein, U. (2009). Exploratory structural equation modeling, integrating CFA and EFA: Application to students' evaluations of university teaching. Structural Equation Modeling, 16, 439-476.

Marsh, H.W., Scalas, L.F., \& Nagengast, B. (2010). Longitudinal tests of competing factor structures for the Rosenberg self-esteem scale: Traits, ephemeral artifacts, and stable response styles. Psychological Assessment, 22, 366-381.

Meyer, J.P., \& Morin, A.J.S. (2016). A person-centered approach to commitment research: Theory, research, and methodology. Journal of Organizational Behavior, 37, 584-612.

Millsap, R. (2011). Statistical approaches to measurement invariance. New York, NY: Taylor \& Francis.

Morin, A.J.S. (2016). Person-centered research strategies in commitment research. In J.P. Meyer (Ed.), The handbook of employee commitment (pp. 490-508). Cheltenham, UK: Edward Elgar.

Morin, A.J.S., Arens, A.K., \& Marsh, H.W. (2015). A bifactor exploratory structural equation modeling framework for the identification of distinct sources of construct-relevant psychometric multidimensionality. Structural Equation Modeling, 1-24.

Morin, A.J.S., Marsh, H.W., \& Nagengast, B. (2013). Chapter 10. Exploratory structural equation modeling. In G.R. Hancock \& R.O. Mueller (Eds), Structural equation modeling: A second course, $2^{\text {nd }}$ edition (pp. 395-436). Charlotte, NC: IAP.

Muthén, L.K., \& Muthén, B.O. (2014). Mplus user guide. Los Angeles, CA: Muthén \& Muthén.

Sass, D.A., \& Schmitt, T.A. (2010). A comparative investigation of rotation criteria within exploratory factor analysis. Multivariate Behavioral Research, 45, 73-103.

Schmitt, T.A., \& Sass, D.A. (2011). Rotation criteria and hypothesis testing for exploratory 
factor analysis: implications for factor pattern loadings and interfactor correlations. Educational \& Psychological Measurement, 71, 95-113. 
SUPPLEMENTS FOR ACADEMIC MOTIVATION PROFILES S30

Table S1

Goodness-of-Fit Statistics of the Measurement Models across Gender

Description $\chi^{2}(d f)$ CFI

TLI RMSEA

$90 \% \mathrm{CI}$

$\Delta \chi^{2}(d f)$

$\triangle$ CFI $\quad \Delta$ TLI $\triangle$ RMSEA

Academic Motivation (Gender)

$798.15^{*}(406)$

.975

.954

.043

$[.038, .047]$

estimated in all groups, no equality constraint)

Loadings (Weak) Invariance

$912.03 *(553)$

$939.76 *(574)$

$1037.48 *(602)$

$1078.58 *(630)$

Latent Variance-Covariance Invariance

Latent Means Invariance $1104.52 *(637)$

$\begin{array}{lll}.977 & .969 & .035\end{array}$

$[.031, .039]$

$[.031, .039]$

$[.033, .041]$

$\begin{array}{lll}.972 & .965 & .037\end{array}$

$.972 \quad .966 \quad .037$

$.033, .040]$

.970

.965

.037

$[.034, .041]$

$-$

(1)

(1)

Covariates (Predictors and Covariate)

Configural Invariance (same model freely

estimated in all groups, no equality constraint)

Loadings (Weak) Invariance

$3280.28 *(1993)$

$.911 \quad .895 \quad .048$

$[.045, .051]$

$3274.74 *(2122) \quad .920 \quad .912 \quad .044 \quad[.041, .047]$

$3347.13 *(2164)$

$\begin{array}{llll}.918 & .911 & .044 & {[.041, .047]}\end{array}$

$3382.38 *(2213)$

$.919 \quad .914 \quad .044$

Uniquenesses (Strict) Invariance

Correlation Uniqueness Invariance

$3401.54 *(2234)$

$\begin{array}{lll}919 & 914 & 043\end{array}$

$3445.95 *(2263)$

$\begin{array}{lll}.918 \quad .915 \quad .043 \\ .917 & .914 & .043\end{array}$

$[.041, .046]$

$[.040, .046]$

$\begin{array}{clll}137.15(147) & +.002 & +.015 & -.008 \\ 26.51(21) & .000 & .000 & .000\end{array}$

$\begin{array}{llll}81.45 *(28) & -.005 & -.004 & +.002\end{array}$

$41.77 *(28) \quad .000+.001 \quad .000$

$27.83 *(7)$

$-.002$

$-.001$

.000

Latent Variance-Covariance Invariance

$3464.64 *(2270)$

$\left.\begin{array}{llll}917 & 914 & .043 & 04041,046\end{array}\right]$

$93.02(129) \quad+.009 \quad+.017 \quad-.004$

$72.76 *(42) \quad-.002 \quad-.001 \quad .000$

$\begin{array}{llll}50.54(49) & +.001 & +.003 & .000\end{array}$

$\begin{array}{llll}22.75(21) & .000 & .000 & -.001\end{array}$

$\begin{array}{llll}44.35^{*}(29) & -.001 & +.001 & .000\end{array}$

Note $* p<01 ; \chi^{2}$. Robust chi-square test of exact fit; $d f$ : Degrees of freedom; CFI: Comparative fit index; TLI: Tucker-Lewis index; RMSEA: Root mean s

approximation; $90 \%$ CI: $90 \%$ confidence interval of the RMSEA; $\Delta \chi^{2}$ : Robust chi-square difference tests; $\Delta$ : Change in relation to the preceding model in the sequence. 
SUPPLEMENTS FOR ACADEMIC MOTIVATION PROFILES S31

Table S2

Goodness-of-Fit Statistics of the Measurement Models across Age

\begin{tabular}{|c|c|c|c|c|c|c|c|c|c|}
\hline Description & $\chi^{2}(d f)$ & CFI & TLI & RMSEA & $90 \% \mathrm{CI}$ & $\Delta \chi^{2}(d f)$ & $\Delta \mathrm{CFI}$ & $\Delta \mathrm{TLI}$ & $\triangle \mathrm{RMSEA}$ \\
\hline \multicolumn{10}{|l|}{ Academic Motivation (Age) } \\
\hline $\begin{array}{l}\text { Configural Invariance (same model freely } \\
\text { estimated in all groups, no equality constraint) }\end{array}$ & $1023.98 *(609)$ & .972 & .948 & .046 & {$[.041, .051]$} & - & - & - & - \\
\hline Loadings (Weak) Invariance & $1307.34 *(903)$ & .973 & .966 & .037 & {$[.033, .042]$} & $310.18(294)$ & +.001 & +.018 & -.009 \\
\hline Intercepts (Strong) Invariance & $1342.43 *(945)$ & .973 & .968 & .036 & {$[.032, .041]$} & $31.69(42)$ & .000 & +.002 & -.001 \\
\hline Uniquenesses (Strict) Invariance & $1389.30 *(1001)$ & .974 & .970 & .035 & {$[.030, .039]$} & $59.06(56)$ & +.001 & +.002 & -.001 \\
\hline Latent Variance-Covariance Invariance & $1453.86 *(1057)$ & .973 & .971 & .034 & {$[.030, .038]$} & $66.02(56)$ & -.001 & +.001 & -.001 \\
\hline Latent Means Invariance & $1488.89 *(1071)$ & .972 & .970 & .035 & {$[.031, .039]$} & $36.97 *(14)$ & -.001 & -.001 & +.001 \\
\hline \multicolumn{10}{|l|}{ Covariates (Predictors and Vitality) } \\
\hline $\begin{array}{l}\text { Configural Invariance (same model freely } \\
\text { estimated in all groups, no equality constraint) }\end{array}$ & $2954.17 *(1993)$ & .925 & .912 & .044 & {$[.040, .047]$} & - & - & - & - \\
\hline Loadings (Weak) Invariance & $3082.83 *(2122)$ & .925 & .917 & .042 & {$[.039, .046]$} & $146.72(129)$ & .000 & +.005 & -.002 \\
\hline Intercepts (Strong) Invariance & $3137.33 *(2164)$ & .924 & .918 & .042 & {$[.039, .045]$} & $54.04(42)$ & -.001 & +.001 & .000 \\
\hline Uniquenesses (Strict) Invariance & $3237.48 *(2213)$ & .920 & .915 & .043 & {$[.040, .046]$} & $89.51 *(49)$ & -.004 & -.003 & +.001 \\
\hline Correlation Uniqueness Invariance & $3296.00 *(2234)$ & .917 & .913 & .043 & {$[.040, .047]$} & $53.60 *(21)$ & -.003 & -.002 & .000 \\
\hline Latent Variance-Covariance Invariance & $3334.19 *(2263)$ & .917 & .913 & .043 & {$[.040, .046]$} & $38.95(29)$ & .000 & .000 & .000 \\
\hline Latent Means Invariance & $3337.48 *(2270)$ & .917 & .914 & .043 & {$[.040, .046]$} & $2.96(7)$ & .000 & +.001 & .000 \\
\hline
\end{tabular}

Note. ${ }^{*} p<.01 ; \chi^{2}$ : Robust chi-square test of exact fit; $d f$ : Degrees of freedom; CFI: Comparative fit index; TLI: Tucker-Lewis index; RMSEA: Root mean square error of approximation; $90 \%$ CI: $90 \%$ confidence interval of the RMSEA; $\Delta \chi^{2}$ : Robust chi-square difference tests; $\Delta$ : Change in relation to the preceding model in the sequence. 
Table S3

Standardized Parameter Estimates from the Final Measurement Models

\begin{tabular}{|c|c|c|c|c|c|c|c|c|c|c|c|c|c|c|}
\hline \multirow[b]{2}{*}{ Items } & \multicolumn{2}{|c|}{ IMKN } & \multicolumn{2}{|c|}{ IMSTI } & \multicolumn{2}{|c|}{ IMACC } & \multicolumn{2}{|c|}{ IDER } & \multicolumn{2}{|c|}{ INJR } & \multicolumn{2}{|c|}{ EXTR } & \multicolumn{2}{|c|}{ AMOT } \\
\hline & $\lambda$ & $\delta$ & $\lambda$ & $\delta$ & $\lambda$ & $\delta$ & $\lambda$ & $\delta$ & $\lambda$ & $\delta$ & $\lambda$ & $\delta$ & $\lambda$ & $\delta$ \\
\hline \multicolumn{15}{|c|}{ Motivation } \\
\hline Item 1 & .32 & .27 & .48 & .51 & .36 & .54 & .72 & .50 & .86 & .26 & .54 & .63 & .86 & .14 \\
\hline Item 2 & .81 & .35 & .88 & .28 & .23 & .30 & .74 & .41 & .22 & .47 & .81 & .32 & .87 & .20 \\
\hline Item 3 & .55 & .42 & .96 & .22 & .72 & .29 & .66 & .53 & .75 & .26 & .67 & .42 & .85 & .15 \\
\hline Item 4 & .66 & .42 & .66 & .35 & .60 & .35 & .70 & .50 & .84 & .15 & .82 & .30 & .89 & .12 \\
\hline & \multicolumn{2}{|c|}{ MINV } & \multicolumn{2}{|c|}{ MAUTS } & \multicolumn{2}{|c|}{ MWAR } & \multicolumn{2}{|c|}{ FINV } & \multicolumn{2}{|c|}{ FAUTS } & \multicolumn{2}{|c|}{ FWAR } & \multicolumn{2}{|c|}{ VITA } \\
\hline Items & $\lambda$ & $\delta$ & $\lambda$ & $\delta$ & $\lambda$ & $\delta$ & $\lambda$ & $\delta$ & $\lambda$ & $\delta$ & $\lambda$ & $\delta$ & $\lambda$ & $\delta$ \\
\hline \multicolumn{15}{|c|}{ Covariates } \\
\hline Item 1 & .56 & .35 & .26 & .47 & .46 & .35 & .64 & .31 & .27 & .41 & .77 & .26 & .65 & .58 \\
\hline Item 2 & .20 & .53 & .49 & .70 & .49 & .36 & .34 & .48 & .43 & .56 & .49 & .37 & .56 & .69 \\
\hline Item 3 & .70 & .35 & .72 & .40 & .23 & .30 & .68 & .36 & .85 & .26 & .39 & .33 & .70 & .51 \\
\hline Item 4 & .30 & .49 & .51 & .32 & .48 & .37 & .42 & .52 & .58 & .33 & .65 & .31 & .86 & .26 \\
\hline Item 5 & .19 & .72 & .69 & .42 & .58 & .44 & .38 & .49 & .92 & .27 & .63 & .36 & .65 & .58 \\
\hline Item 6 & .55 & .38 & .69 & .43 & .57 & .41 & .46 & .37 & .69 & .36 & .71 & .33 & .59 & .65 \\
\hline Item 7 & - & - & .70 & .47 & - & - & - & - & .70 & .40 & - & - & .86 & .26 \\
\hline Item 8 & - & - & .55 & .47 & - & - & - & - & .52 & .37 & - & - & - & - \\
\hline Item 9 & - & - & .13 & .63 & - & - & - & - & .17 & .65 & - & - & - & - \\
\hline
\end{tabular}

Note $\lambda=$ factor loading; $\delta=$ item uniqueness; IMKN = intrinsic motivation to know; IMSTI = intrinsic motivation to experience stimulation; IMACC = intrinsic motivation to accomplish; IDER = identified regulation; INJR = introjected regulation; EXTR = external regulation; AMOT = amotivation; MINV = mother involvement; MAUTS = mother autonomy support; MWAR = mother warmth; FINV = father involvement;

FAUTS $=$ father autonomy support FWAR = father warmth; VITA = vitality. 
SUPPLEMENTS FOR ACADEMIC MOTIVATION PROFILES S33

Table S4

Goodness-of-Fit Results From the Latent Profile Analyses Across Gender

\begin{tabular}{|c|c|c|c|c|c|c|c|c|c|c|}
\hline Model & LL & $\# \mathrm{fp}$ & Scaling & AIC & CAIC & BIC & ABIC & Entropy & aLMR & BLRT \\
\hline \multicolumn{11}{|l|}{ Males } \\
\hline 1 profile & -3873.55 & 21 & 1.06 & 7789.11 & 7895.93 & 7874.93 & 7808.29 & $\mathrm{Na}$ & $\mathrm{Na}$ & $\mathrm{Na}$ \\
\hline 2 profile & -3517.46 & 36 & 1.17 & 7106.93 & 7290.05 & 7254.05 & 7139.81 & 0.89 & $\leq .001$ & $\leq .001$ \\
\hline 3 profile & -3279.42 & 51 & 1.23 & 6660.83 & 6920.26 & 6869.26 & 6707.41 & 0.92 & $\leq .001$ & $\leq .001$ \\
\hline 4 profile & -3175.37 & 66 & 1.27 & 6482.74 & 6818.47 & 6752.47 & 6543.02 & 0.92 & .076 & $\leq .001$ \\
\hline 5 profile & -3065.43 & 81 & 1.20 & 6292.86 & 6704.89 & 6623.89 & 6366.84 & 0.90 & .017 & $\leq .001$ \\
\hline 6 profile & -2963.23 & 96 & 1.18 & 6118.46 & 6606.79 & 6510.79 & 6206.13 & 0.91 & .027 & $\leq .001$ \\
\hline 7 profile & -2919.00 & 111 & 1.16 & 6060.00 & 6624.63 & 6513.63 & 6161.37 & 0.90 & .531 & $\leq .001$ \\
\hline 8 profile & -2881.53 & 126 & 1.13 & 6015.06 & 6655.99 & 6529.99 & 6130.13 & 0.91 & .209 & $\leq .001$ \\
\hline \multicolumn{11}{|l|}{ Females } \\
\hline 1 profile & -5276.41 & 21 & 1.15 & 10594.81 & 10708.73 & 10687.73 & 10621.06 & $\mathrm{Na}$ & $\mathrm{Na}$ & $\mathrm{Na}$ \\
\hline 2 profile & -4676.42 & 36 & 1.40 & 9424.85 & 9620.14 & 9584.14 & 9469.85 & 0.97 & $\leq .001$ & $\leq .001$ \\
\hline 3 profile & -4327.58 & 51 & 1.59 & 8757.16 & 9033.82 & 8982.82 & 8820.91 & 0.91 & .167 & $\leq .001$ \\
\hline 4 profile & -4183.62 & 66 & 1.32 & 8499.23 & 8857.27 & 8791.27 & 8581.73 & 0.92 & .013 & $\leq .001$ \\
\hline 5 profile & -4039.63 & 81 & 1.39 & 8241.27 & 8680.68 & 8599.68 & 8342.52 & 0.88 & .272 & $\leq .001$ \\
\hline 6 profile & -3934.06 & 96 & 1.261 & 8060.12 & 8580.90 & 8484.90 & 8180.12 & 0.90 & .178 & $\leq .001$ \\
\hline 7 profile & -3845.02 & 111 & 1.21 & 7912.04 & 8514.20 & 8403.20 & 8050.80 & 0.90 & .154 & $\leq .001$ \\
\hline 8 profile & -3759.20 & 126 & 1.351 & 7770.40 & 8453.93 & 8327.93 & 7927.90 & 0.90 & .473 & $\leq .001$ \\
\hline
\end{tabular}

Note. LL: Model LogLikelihood; \#fp: Number of free parameters; Scaling = Scaling factor associated with MLR loglikelihood estimates; AIC: Akaïke Information Criteria; CAIC: Constant AIC; BIC: Bayesian Information Criteria; ABIC: Sample-Size Adjusted BIC; aLMR: Adjusted Lo-Mendell-Rubin likelihood ratio test; BLRT: Bootstrap Likelihood Ratio Test; LRT: Likelihood Ratio Test; df: Degrees of Freedom associated with the LRT; M: Means; V: Variances; P: Class probabilities. 
Table S5

Goodness-of-Fit Results from the Latent Profile Analyses across Age Group

\begin{tabular}{|c|c|c|c|c|c|c|c|c|c|c|}
\hline Model & LL & $\#$ fp & Scaling & AIC & CAIC & BIC & ABIC & Entropy & aLMR & BLRT \\
\hline \multicolumn{11}{|l|}{17 to 20} \\
\hline 1 profile & -3240.21 & 21 & 1.19 & 6522.43 & 6625.84 & 6604.84 & 6538.21 & $\mathrm{Na}$ & $\mathrm{Na}$ & $\mathrm{Na}$ \\
\hline 2 profile & -2861.49 & 36 & 1.25 & 5794.99 & 5972.26 & 5936.26 & 5822.04 & 0.92 & $\leq .001$ & $\leq .001$ \\
\hline 3 profile & -2658.05 & 51 & 1.32 & 5418.10 & 5669.24 & 5618.24 & 5456.43 & 0.90 & .016 & $\leq .001$ \\
\hline 4 profile & -2558.38 & 66 & 1.23 & 5248.76 & 5573.76 & 5507.76 & 5298.36 & 0.85 & .059 & $\leq .001$ \\
\hline 5 profile & -2463.17 & 81 & 1.27 & 5088.34 & 5487.21 & 5406.21 & 5149.22 & 0.89 & .192 & $\leq .001$ \\
\hline 6 profile & -2381.48 & 96 & 1.26 & 4954.96 & 5427.69 & 5331.69 & 5027.11 & 0.88 & .884 & $\leq .001$ \\
\hline 7 profile & -2317.75 & 111 & 1.14 & 4857.51 & 5404.10 & 5293.10 & 4940.93 & 0.91 & .192 & $\leq .001$ \\
\hline 8 profile & -2259.17 & 126 & 1.26 & 4770.34 & 5390.79 & 5264.79 & 4865.03 & 0.91 & .727 & $\leq .001$ \\
\hline \multicolumn{11}{|l|}{21 to 23} \\
\hline 1 profile & -2981.03 & 21 & 1.08 & 6004.07 & 6105.96 & 6084.96 & 6018.34 & $\mathrm{Na}$ & $\mathrm{Na}$ & $\mathrm{Na}$ \\
\hline 2 profile & -2640.49 & 36 & 1.36 & 5352.99 & 5527.67 & 5491.67 & 5377.46 & 0.96 & .004 & $\leq .001$ \\
\hline 3 profile & -2464.32 & 51 & 1.31 & 5030.64 & 5278.11 & 5227.11 & 5065.32 & 0.92 & .017 & $\leq .001$ \\
\hline 4 profile & -2363.34 & 66 & 1.28 & 4858.69 & 5178.93 & 5112.93 & 4903.56 & 0.94 & .194 & $\leq .001$ \\
\hline 5 profile & -2279.70 & 81 & 1.13 & 4721.40 & 5114.43 & 5033.43 & 4776.47 & 0.92 & .055 & $\leq .001$ \\
\hline 6 profile & -2212.37 & 96 & 1.15 & 4616.74 & 5082.55 & 4986.55 & 4682.01 & 0.92 & .091 & $\leq .001$ \\
\hline 7 profile & -2163.91 & 111 & 1.10 & 4549.82 & 5088.42 & 4977.42 & 4625.29 & 0.90 & .106 & $\leq .001$ \\
\hline 8 profile & -2117.37 & 126 & 1.07 & 4486.75 & 5098.12 & 4972.12 & 4572.41 & 0.91 & .025 & $\leq .001$ \\
\hline \multicolumn{11}{|l|}{24 or more } \\
\hline 1 profile & -2105.38 & 21 & 1.12 & 4252.76 & 4346.94 & 4325.94 & 4259.37 & $\mathrm{Na}$ & $\mathrm{Na}$ & $\mathrm{Na}$ \\
\hline 2 profile & -1885.57 & 36 & 1.14 & 3843.13 & 4004.58 & 3968.58 & 3854.47 & 0.96 & $\leq .001$ & $\leq .001$ \\
\hline 3 profile & -1745.16 & 51 & 1.13 & 3592.32 & 3821.04 & 3770.04 & 3608.38 & 0.92 & $\leq .001$ & $\leq .001$ \\
\hline 4 profile & -1673.28 & 66 & 1.13 & 3478.57 & 3774.56 & 3708.56 & 3499.36 & 0.93 & .036 & $\leq .001$ \\
\hline 5 profile & -1618.31 & 81 & 1.08 & 3398.61 & 3761.88 & 3680.88 & 3424.13 & 0.90 & .051 & $\leq .001$ \\
\hline 6 profile & -1572.02 & 96 & 1.03 & 3336.05 & 3766.59 & 3670.59 & 3366.29 & 0.91 & .764 & $\leq .001$ \\
\hline 7 profile & -1526.32 & 111 & 0.99 & 3274.64 & 3772.45 & 3661.45 & 3309.61 & 0.91 & .223 & $\leq .001$ \\
\hline 8 profile & -1496.79 & 126 & 1.08 & 3245.58 & 3810.67 & 3684.67 & 3285.27 & 0.92 & .623 & $\leq .001$ \\
\hline
\end{tabular}

Note. LL: Model LogLikelihood; \#fp: Number of free parameters; Scaling = Scaling factor associated with MLR loglikelihood estimates; AIC: Akaïke Information Criteria; CAIC: Constant AIC; BIC: Bayesian Information Criteria; ABIC: Sample-Size Adjusted BIC; aLMR: Adjusted Lo-Mendell-Rubin likelihood ratio test; BLRT: Bootstrap Likelihood Ratio Test;

LRT: Likelihood Ratio Test; df: Degrees of Freedom associated with the LRT; M: Means; V: Variances; P: Class probabilities. 
SUPPLEMENTS FOR ACADEMIC MOTIVATION PROFILES S35

Table S6

Detailed Results from the Final LPA Solution of Distributional Similarity.

\begin{tabular}{|c|c|c|c|c|c|c|}
\hline \multirow[b]{2}{*}{ Type of regulation } & \multicolumn{2}{|c|}{ Profile 1} & \multicolumn{2}{|l|}{ Profile 2} & \multicolumn{2}{|c|}{ Profile 3} \\
\hline & Mean $(\mathrm{CI})$ & Variance & Mean $(\mathrm{CI})$ & Variance & Mean $(\mathrm{CI})$ & Variance \\
\hline IMKN & $0.49(0.34 ; 0.64)$ & 0.14 & $0.15(-0.07 ; 0.37)$ & 0.10 & $0.58(0.41 ; 0.75)$ & 0.21 \\
\hline IMSTI & $-0.31(-0.48 ;-0.15)$ & 0.37 & $-0.22(-0.38 ;-0.07)$ & 0.18 & $-0.17(-0.36 ; 0.01)$ & 0.38 \\
\hline IMACC & $0.15(-0.05 ; 0.35)$ & 0.26 & $0.18(-0.04 ; 0.40)$ & 0.09 & $0.63(0.46 ; 0.81)$ & 0.15 \\
\hline IDER & $-0.23(-0.42 ;-0.04)$ & 0.53 & $-0.04(-0.27 ; 0.19)$ & 0.36 & $0.79(0.70 ; 0.87)$ & 0.06 \\
\hline INJR & $0.07(-0.12 ; 0.26)$ & 0.57 & $0.60(0.42 ; 0.78)$ & 0.23 & $0.93(0.79 ; 1.08)$ & 0.23 \\
\hline EXTR & $-0.20(-0.42 ; 0.01)$ & 0.76 & $0.37(0.22 ; 0.51)$ & 0.28 & $1.04(1.00 ; 1.09)$ & 0.03 \\
\hline \multirow[t]{3}{*}{ AMOT } & $-0.97(-0.98 ;-0.96)$ & 0.00 & $-0.43(-0.58 ;-0.29)$ & 0.16 & $-0.89(-0.92 ;-0.87)$ & 0.01 \\
\hline & \multicolumn{2}{|c|}{ Profile 4} & \multicolumn{2}{|l|}{ Profile 5} & \multicolumn{2}{|l|}{-} \\
\hline & Mean $(\mathrm{CI})$ & Variance & Mean $(\mathrm{CI})$ & Variance & - & - \\
\hline IMKN & $-0.77(-0.92 ;-0.62)$ & 0.39 & $-0.11(-0.48 ; 0.26)$ & 0.28 & - & - \\
\hline IMSTI & $-0.12(-0.39 ; 0.16)$ & 0.33 & $1.05(0.77 ; 1.34)$ & 0.11 & - & - \\
\hline IMACC & $-0.56(-0.70 ;-0.42)$ & 0.35 & $-0.17(-0.46 ; 0.12)$ & 0.39 & - & - \\
\hline IDER & $-0.64(-0.88 ;-0.39)$ & 1.11 & $0.59(0.26 ; 0.91)$ & 0.31 & - & - \\
\hline INJR & $-0.36(-0.69 ;-0.03)$ & 0.53 & $-1.35(-1.47 ;-1.23)$ & 0.06 & - & - \\
\hline EXTR & $-0.81(-1.00 ;-0.62)$ & 0.77 & $-0.08(-0.42 ; 0.27)$ & 0.45 & - & - \\
\hline AMOT & $0.68(0.38 ; 0.98)$ & 0.32 & $1.53(1.38 ; 1.68)$ & 0.05 & - & - \\
\hline
\end{tabular}

Note. Profile indicators are factor scores estimated with a mean of 0 and a standard deviation of 1 in the total sample; $\mathrm{CI}=95 \%$ confidence interval; $\mathrm{IMKN}=$ intrinsic motivation to know; IMSTI = intrinsic motivation to experience stimulation; IMACC $=$ intrinsic motivation to accomplish; IDER $=$ identified regulation; INJR = introjected regulation; EXTR = external regulation; AMOT = amotivation; VITA = vitality; MINV = mother involvement; MAUTS = mother autonomy support; MWAR = mother warmth; FINV = father involvement; FAUTS = father autonomy support; FWAR = father warmth; Profile 1: Knowledge-Oriented; Profile 2: Controlled; Profile 3: Multifaceted; Profile 4: Unmotivated; Profile 5: Hedonist. 
SUPPLEMENTS FOR ACADEMIC MOTIVATION PROFILES S36

Table S7

Means on the Oucome (Vitality) for each Profile and Mean Differences between Profiles.

\begin{tabular}{|c|c|c|c|c|c|}
\hline Profile & Mean $(\mathrm{CI})$ & $\Delta(\mathrm{SE})$ vs. Profile 2 & $\Delta(\mathrm{SE})$ vs. Profile 3 & $\Delta(\mathrm{SE})$ vs. Profile 4 & $\Delta(\mathrm{SE})$ vs. Profile 5 \\
\hline Profile 1 & $0.30(0.07 ; 0.54)$ & $0.44(0.14)^{* *}$ & $0.18(0.16)$ & $0.56(0.15)^{* *}$ & $-1.65(0.12)^{* *}$ \\
\hline Profile 2 & $-0.14(-0.26 ;-0.02)$ & - & $-0.26(0.12)^{*}$ & $0.12(0.12)$ & $-2.09(0.06)^{* *}$ \\
\hline Profile 3 & $0.12(-0.07 ; 0.31)$ & & - & $0.38(0.14)^{* *}$ & $-1.83(0.10)^{* *}$ \\
\hline Profile 4 & $-0.26(-0.45 ;-0.07)$ & & & - & $-2.21(0.10)^{* *}$ \\
\hline
\end{tabular}

Note. ${ }^{*} p<.05 ; * * p<.01$; Vitality means are based on factor scores estimated with a mean of 0 and a standard deviation of 1 in the total sample, so that differences across profiles can be interpreted in standardized units; $\mathrm{CI}=95 \%$ confidence interval; $\Delta$ (SE): Mean difference across profiles and their standard erros calculated using the multivariate delta method (Raykov \& Marcoulides, 2004); Profile 1: Knowledge-Oriented; Profile 2: Controlled; Profile 3: Multifaceted; Profile 4: Unmotivated; Profile 5: Hedonist. 


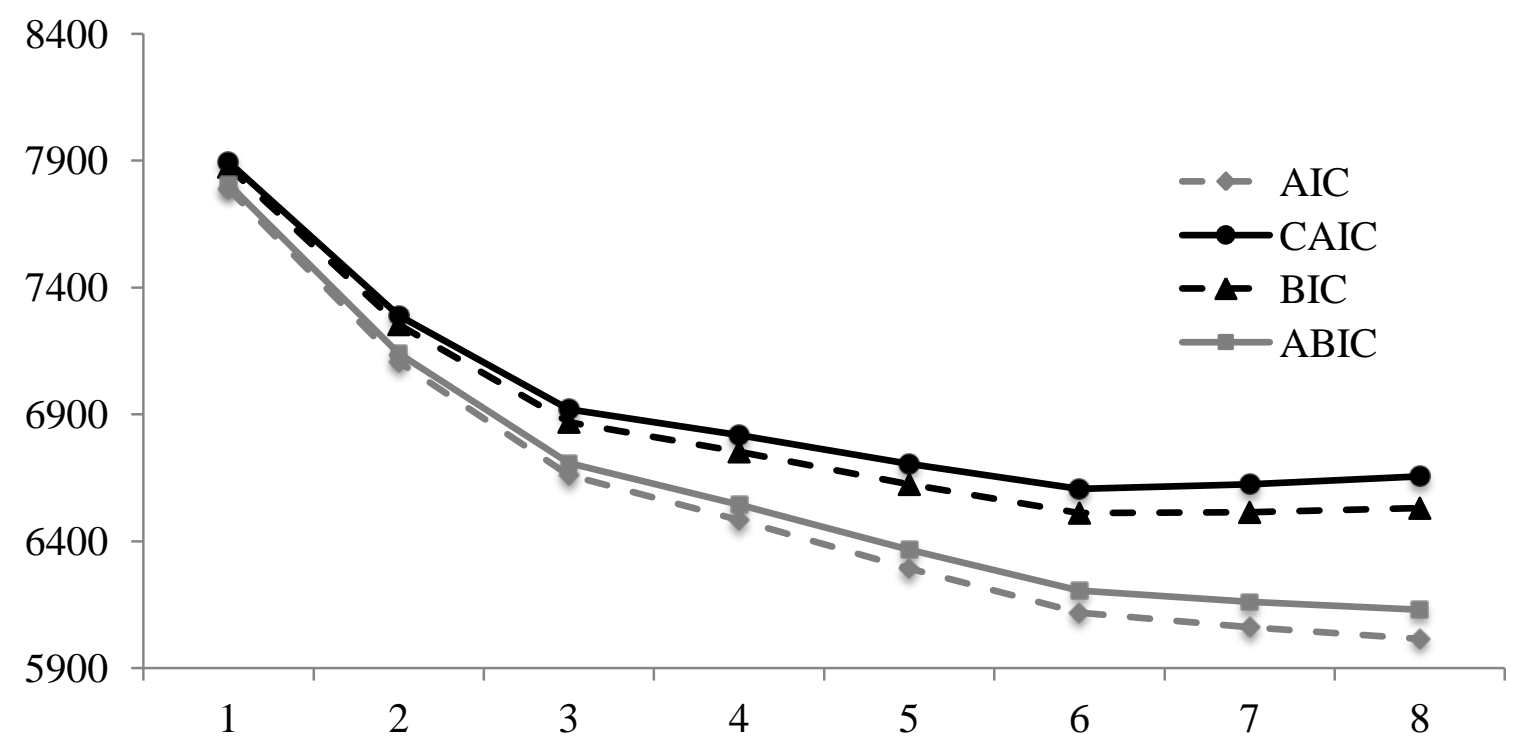

Figure S1. Elbow Plot of the Information Criteria for the Latent Profile Analyses (Males). 


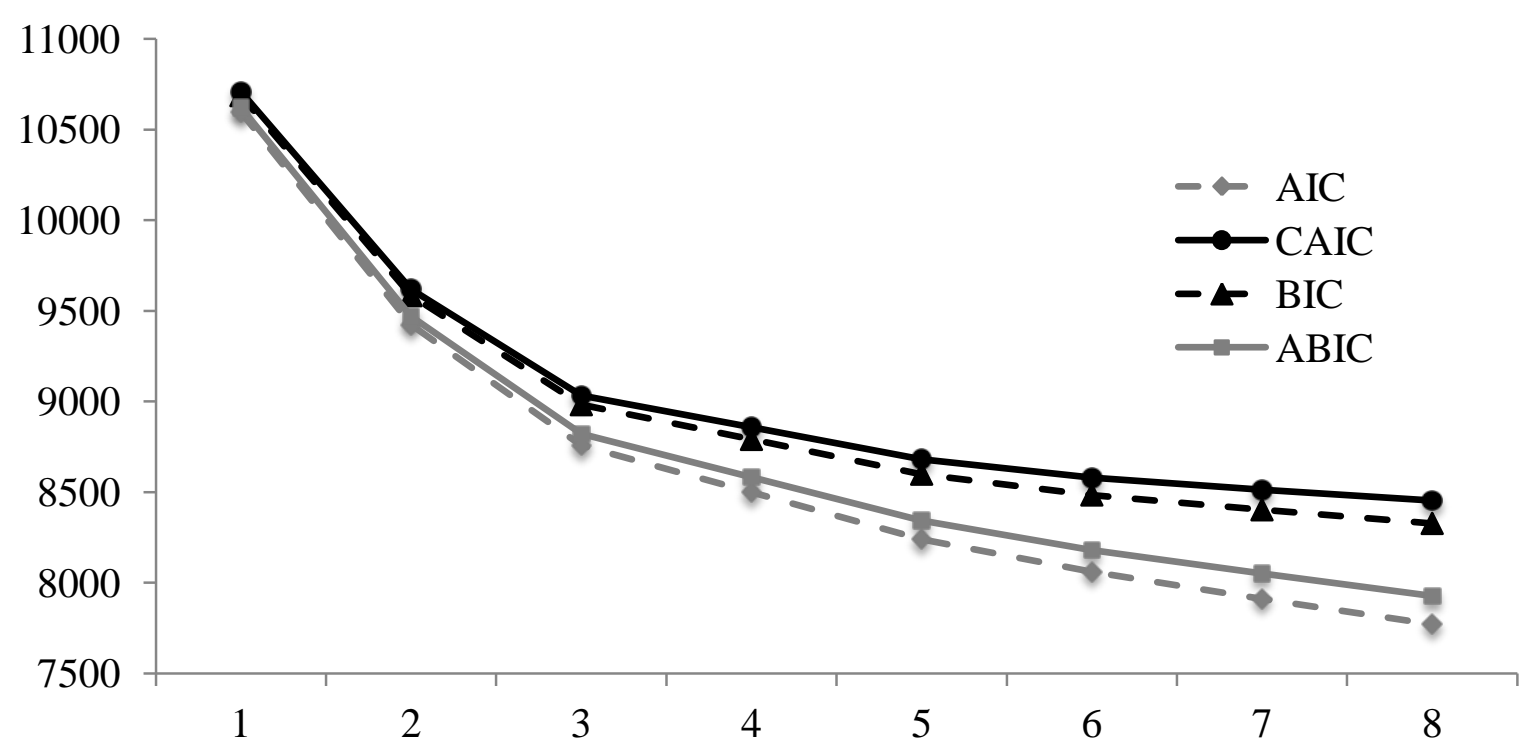

Figure S2. Elbow Plot of the Information Criteria for the Latent Profile Analyses (Females). 


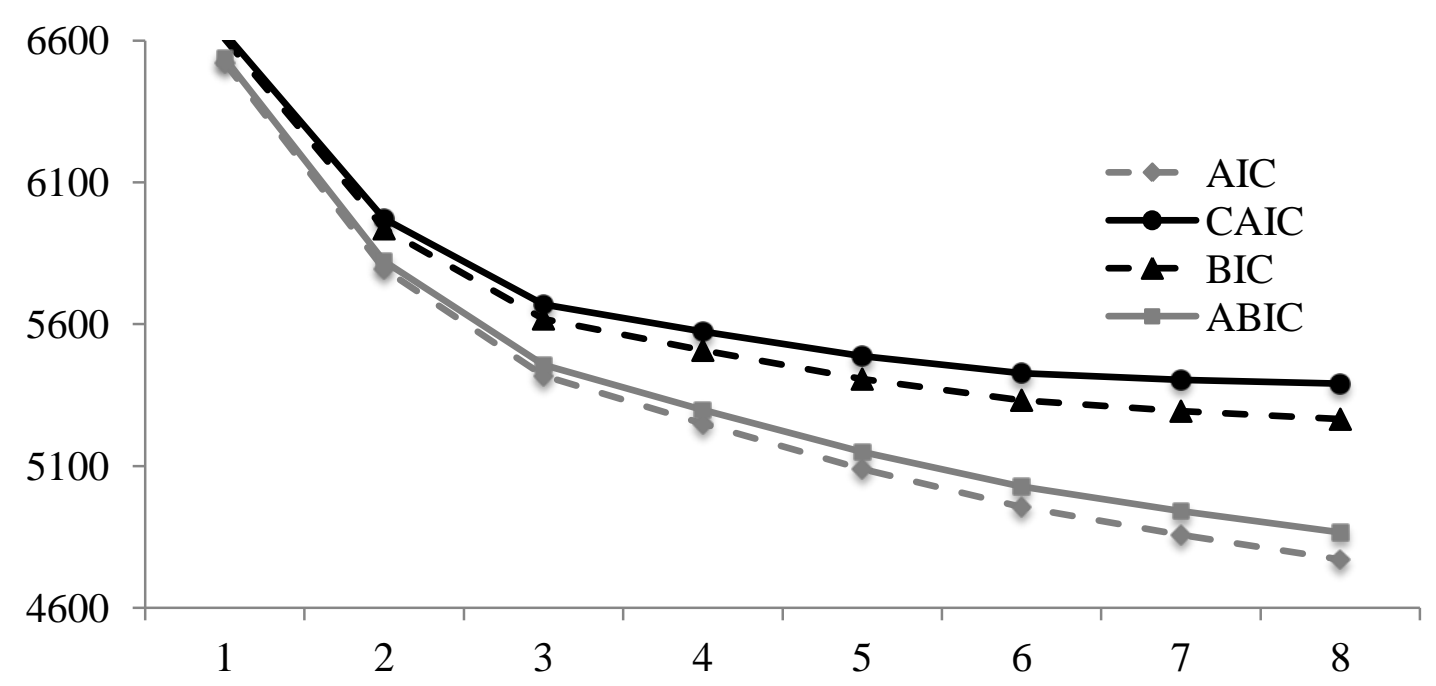

Figure S3. Elbow Plot of the Information Criteria for the Latent Profile Analyses (17 to 20 years old).

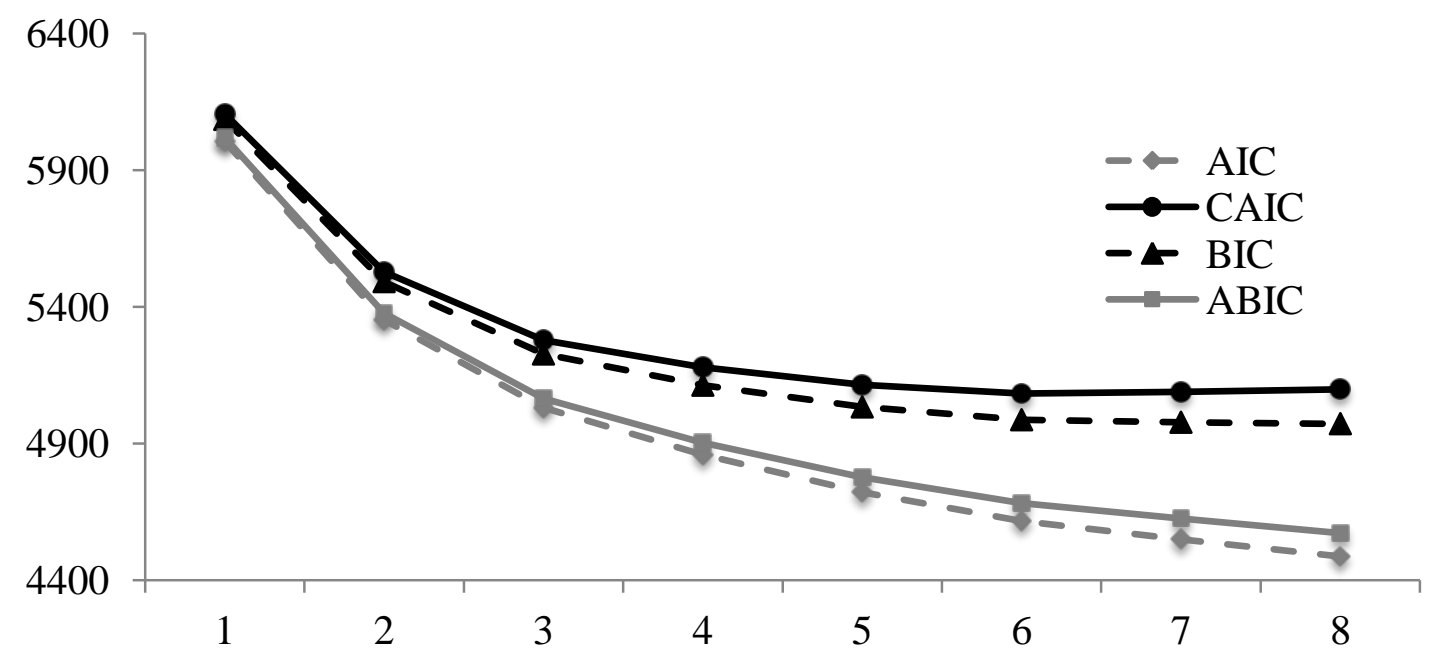


Figure S4. Elbow Plot of the Information Criteria for the Latent Profile Analyses (21 to 23 years old).

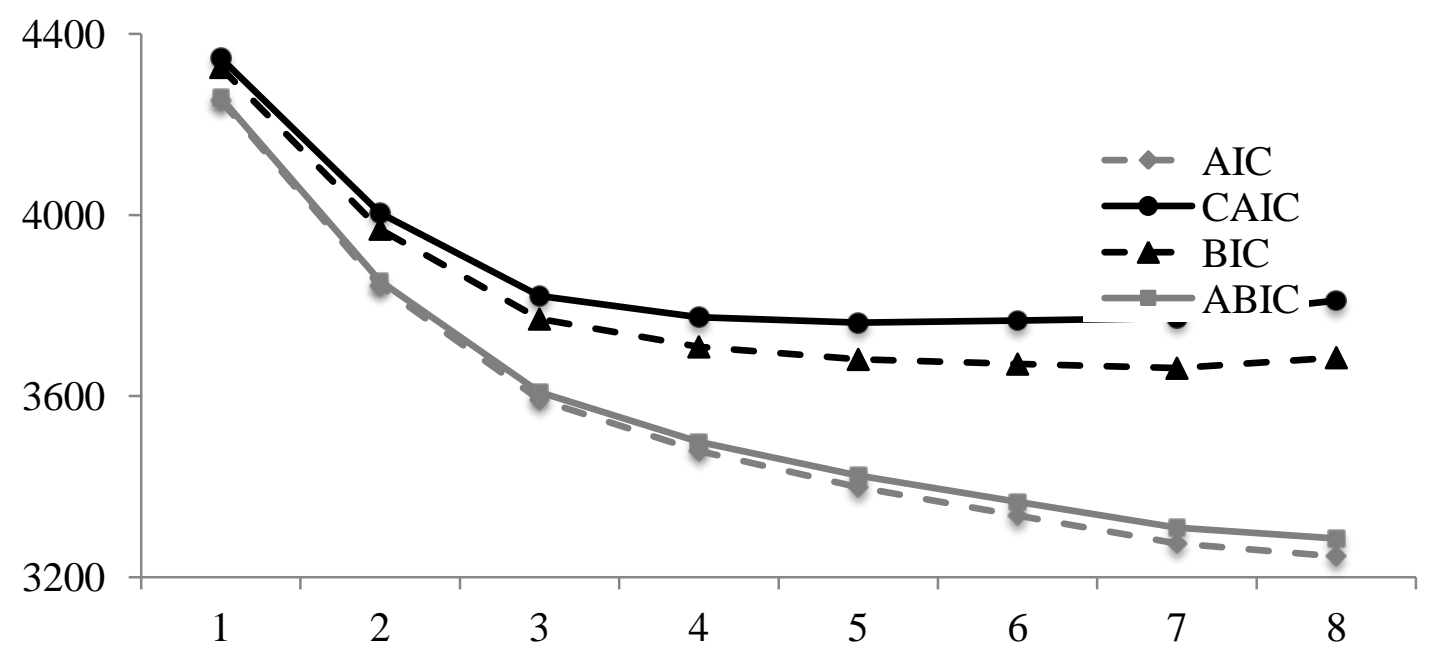

Figure S5. Elbow Plot of the Information Criteria for the Latent Profile Analyses (24 years old or more). 
TITLE: Mplus Input Code to Estimate a 5-Class Latent Profile Solution Without Predictors and Covariate in a Single Groups.

! In all input files, statements preceded by! are annotations.

! Use the following statement to identify the data set. Here, the data set is labelled FSCORES.dat.

DATA: FILE IS FSCORES.dat;

! The variables names function identifies all variables in the data set, in order of appearance,

! whereas the usevar command identifies the variables used in the analysis.

VARIABLE:

NAMES = ID GENDER AGEG3 IMKNOW IMSTI IMACC IDER INJR EXTR AMOT

VITAL MINVOLV MAUTOSUP MWARM FINVOLV PAUTOSUP FWARM;

USEVARIABLES = IMKNOW IMSTI IMACC IDER INJR EXTR AMOT;

! To indicate the missing data code used for all variables

MISSING ARE ALL *;

! The variables GENDER or AGEG3 are the grouping variables. Here, we select the gender group

! with a value of 1 (i.e., Males).

\section{USEOBS GENDER EQ 1;}

! To estimate the model in the FEMALE sample, replace this statement by:

! USEOBS GENDER EQ 2;

! To estimate the model in the YOUNGEST sample, replace this statement by:

! USEOBS AGEG3 EQ 1;

! To estimate the model in the MIDDLE age sample, replace this statement by:

! USEOBS AGEG3 EQ 2;

! To estimate the model in the OLDEST age sample, replace this statement by:

! USEOBS AGEG3 EQ 2;

! The following identifies the number of latent profiles requested in the analysis.

CLASSES $=\mathrm{c}(5)$

! The next section defines the analysis.

! By default, the Robust Maximum Likelihood (MLR) estimator is used.

ANALYSIS:

ESTIMATOR $=$ MLR;

! To request the use of multiple processors.

Process $=3$;

! The following set up is to estimate the model using 3 processors, 5000 starts values, 200 final stage optimizations, and 100 iterations, 
TYPE $=$ MIXTURE; STARTS $=5000$ 200; STITERATIONS $=100 ;$

! The following are to increase the defaults starts and iteration for the BLRT.

LRTBOOTSTRAP = 100; LRTSTARTS = $1058020 ;$

! The next statement defines the model. Here, a simple latent profile model is specified with variances equal across profiles. MODEL:

! In this input, the overall model statement would define sections of the models that are common

! across profiles.

! It is in this section that the factor mixture specification is indicated, with a single factor added to

! control for the commonality shared among all indicators (global levels of motivation).

! The \%c\#1\% to \%c\#5\% sections are class-specific statement with which to specify which part of the

$!$ model is freely estimated in each profile.

! For a simple latent profile model, include the means of the indicators (using []) in all profiles.

! To also freely estimate all variances, add the following in each class-specific statement:

! IMKNOW IMSTI IMACC IDER INJR EXTR AMOT;

\%OVERALL\%

FG BY IMKNOW* IMSTI IMACC IDER INJR EXTR AMOT;

FG@1;

[FG@0];

$\%$ c\#1\%

[IMKNOW IMSTI IMACC IDER INJR EXTR AMOT];

IMKNOW IMSTI IMACC IDER INJR EXTR AMOT;

$\% \mathrm{c \# 2} \%$

[IMKNOW IMSTI IMACC IDER INJR EXTR AMOT];

IMKNOW IMSTI IMACC IDER INJR EXTR AMOT;

$\% \mathrm{c} \# 3 \%$

[IMKNOW IMSTI IMACC IDER INJR EXTR AMOT];

IMKNOW IMSTI IMACC IDER INJR EXTR AMOT;

$\% \mathrm{c} \# 4 \%$

[IMKNOW IMSTI IMACC IDER INJR EXTR AMOT];

IMKNOW IMSTI IMACC IDER INJR EXTR AMOT;

$\% \mathrm{c} \# 5 \%$

[IMKNOW IMSTI IMACC IDER INJR EXTR AMOT]; 
IMKNOW IMSTI IMACC IDER INJR EXTR AMOT;

OUTPUT:

STDYX SAMPSTAT CINTERVAL SVALUES RESIDUAL TECH1 TECH7 TECH11 TECH14; 
TITLE: Mplus Input Code to Estimate a Configural Similarity Model for a Latent Profile Solution Without Predictors and Covariates

! Annotations only focus on functions not previously defined.

! The variables GENDER or AGEG3 are the grouping variables.

! Here, we focus this illustration on the gender groups

! To estimate a multiple group latent profile model, we identify the groups using the

! KNOWNCLASS function to create a latent categorical variable reflecting these groups.

! Here, we label this variable $C G$ and then define the 2 values of $C G$ (based on the value of the

$!$ observed variable GENDER where $1=$ Males and $2=$ Females).

! Then the CLASSES function is used to label all latent categorical variables: CG (previously defined,

! with 2 levels, based on the observed grouping variable), and $C$ (the latent profiles)

KNOWNCLASS $=$ CG $($ GENDER $=1$ GENDER $=2)$;

CLASSES = CG (2) C (5);

ANALYSIS:

ESTIMATOR $=$ MLR; Process $=3$;

TYPE $=$ MIXTURE; STARTS $=5000$ 200; STITERATIONS = 100;

MODEL:

\%OVERALL\%

FG BY IMKNOW* IMSTI IMACC IDER INJR EXTR AMOT;

FG@1; [FG@0];

! The following statements in the \%OVERALL\% section indicate that the class sizes (class

! probabilities) are freely estimated in all samples.

! Only k-1 statements are required (i.e. 4 for a 5 class model)

c\#1 on cg\#1; c\#2 on cg\#1; c\#3 on cg\#1; c\#4 on cg\#1;

! Class specific statements now need to be defined using a combination of both the known classes CG

! and the estimated classes $C$.

! Labels in parentheses identify parameters that are estimated to be equal.

! Lists of constraints (e.g., m1-m3) apply to the parameters in order of appearance (e.g., mmale11

! applies to IMSTI, mmale2 to IMACC, and so on.

$\%$ cg\#1.c\#1\%

FG by IMKNOW* (fg1)

IMSTI IMACC IDER INJR EXTR AMOT(fg2-fg7);

[IMKNOW IMSTI IMACC IDER INJR EXTR AMOT](mmale1-mmale7); 
IMKNOW IMSTI IMACC IDER INJR EXTR AMOT(vmale1-vmale7);

$\%$ cg\#1.c\#2\%

FG by IMKNOW* (fg1)

IMSTI IMACC IDER INJR EXTR AMOT(fg2-fg7);

[IMKNOW IMSTI IMACC IDER INJR EXTR AMOT](mmale8-mmale14);

IMKNOW IMSTI IMACC IDER INJR EXTR AMOT(vmale8-vmale14);

$\%$ cg\#1.c\#3\%

FG by IMKNOW* (fg1)

IMSTI IMACC IDER INJR EXTR AMOT(fg2-fg7);

[IMKNOW IMSTI IMACC IDER INJR EXTR AMOT](mmale15-mmale21);

IMKNOW IMSTI IMACC IDER INJR EXTR AMOT(vmale15-vmale21);

$\%$ cg\#1.c\#4\%

FG by IMKNOW* (fg1)

IMSTI IMACC IDER INJR EXTR AMOT(fg2-fg7);

[IMKNOW IMSTI IMACC IDER INJR EXTR AMOT](mmale22-mmale28);

IMKNOW IMSTI IMACC IDER INJR EXTR AMOT(vmale22-vmale28);

$\%$ cg\#1.c\#5\%

FG by IMKNOW* (fg1)

IMSTI IMACC IDER INJR EXTR AMOT(fg2-fg7);

[IMKNOW IMSTI IMACC IDER INJR EXTR AMOT](mmale29-mmale35);

IMKNOW IMSTI IMACC IDER INJR EXTR AMOT(vmale29-vmale35);

$\%$ cg\#2.c\#1\%

FG by IMKNOW* (fg1)

IMSTI IMACC IDER INJR EXTR AMOT(fg2-fg7);

[IMKNOW IMSTI IMACC IDER INJR EXTR AMOT](mfem1-mfem7);

IMKNOW IMSTI IMACC IDER INJR EXTR AMOT(vfem1-vfem7);

$\%$ cg\#2.c\#2\%

FG by IMKNOW* (fg1)

IMSTI IMACC IDER INJR EXTR AMOT(fg2-fg7);

[IMKNOW IMSTI IMACC IDER INJR EXTR AMOT](mfem8-mfem14);

IMKNOW IMSTI IMACC IDER INJR EXTR AMOT(vfem8-vfem14); 
$\%$ cg\#2.c\#3\%

FG by IMKNOW* (fg1)

IMSTI IMACC IDER INJR EXTR AMOT(fg2-fg7);

[IMKNOW IMSTI IMACC IDER INJR EXTR AMOT](mfem15-mfem21);

IMKNOW IMSTI IMACC IDER INJR EXTR AMOT(vfem15-vfem21);

$\%$ cg\#2.c\#4\%

FG by IMKNOW* (fg1)

IMSTI IMACC IDER INJR EXTR AMOT(fg2-fg7);

[IMKNOW IMSTI IMACC IDER INJR EXTR AMOT](mfem22-mfem28);

IMKNOW IMSTI IMACC IDER INJR EXTR AMOT(vfem22-vfem28);

$\%$ cg\#2.c\#5\%

FG by IMKNOW* (fg1)

IMSTI IMACC IDER INJR EXTR AMOT(fg2-fg7);

[IMKNOW IMSTI IMACC IDER INJR EXTR AMOT](mfem29-mfem35);

IMKNOW IMSTI IMACC IDER INJR EXTR AMOT(vfem29-vfem35);

OUTPUT:

STDYX SAMPSTAT CINTERVAL SVALUES RESIDUAL TECH1 TECH7; 
TITLE: Mplus Input Code to Estimate a Structural Similarity Model for a Latent Profile Solution Without Predictors and Covariates.

! Annotations only focus on functions not previously defined.

\%OVERALL\%

FG BY IMKNOW* IMSTI IMACC IDER INJR EXTR AMOT; FG@1; [FG@0];

c\#1 on cg\#1; c\#2 on cg\#1; c\#3 on cg\#1; c\#4 on cg\#1;

! Here, the means are freely estimated in all profiles but constrained to be equal across gender.

$\%$ cg\#1.c\#1\%

FG by IMKNOW* (fg1)

IMSTI IMACC IDER INJR EXTR AMOT(fg2-fg7);

[IMKNOW IMSTI IMACC IDER INJR EXTR AMOT](mmale1-mmale7);

IMKNOW IMSTI IMACC IDER INJR EXTR AMOT(vmale1-vmale7);

$\%$ cg\#1.c\#2\%

FG by IMKNOW* (fg1)

IMSTI IMACC IDER INJR EXTR AMOT(fg2-fg7);

[IMKNOW IMSTI IMACC IDER INJR EXTR AMOT](mmale8-mmale14);

IMKNOW IMSTI IMACC IDER INJR EXTR AMOT(vmale8-vmale14);

$\%$ cg\#1.c\#3\%

FG by IMKNOW* (fg1)

IMSTI IMACC IDER INJR EXTR AMOT(fg2-fg7);

[IMKNOW IMSTI IMACC IDER INJR EXTR AMOT](mmale15-mmale21);

IMKNOW IMSTI IMACC IDER INJR EXTR AMOT(vmale15-vmale21);

$\%$ cg\#1.c\#4\%

FG by IMKNOW* (fg1)

IMSTI IMACC IDER INJR EXTR AMOT(fg2-fg7);

[IMKNOW IMSTI IMACC IDER INJR EXTR AMOT](mmale22-mmale28);

IMKNOW IMSTI IMACC IDER INJR EXTR AMOT(vmale22-vmale28);

$\%$ cg\#1.c\#5\%

FG by IMKNOW* (fg1)

IMSTI IMACC IDER INJR EXTR AMOT(fg2-fg7);

[IMKNOW IMSTI IMACC IDER INJR EXTR AMOT](mmale29-mmale35);

IMKNOW IMSTI IMACC IDER INJR EXTR AMOT(vmale29-vmale35);

$\%$ cg\#2.c\#1\%

FG by IMKNOW* (fg1)

IMSTI IMACC IDER INJR EXTR AMOT(fg2-fg7);

[IMKNOW IMSTI IMACC IDER INJR EXTR AMOT](mmale1-mmale7);

IMKNOW IMSTI IMACC IDER INJR EXTR AMOT(vfem1-vfem7);

$\%$ cg\#2.c\#2\%

FG by IMKNOW* (fg1)

IMSTI IMACC IDER INJR EXTR AMOT(fg2-fg7);

[IMKNOW IMSTI IMACC IDER INJR EXTR AMOT](mmale8-mmale14); 
IMKNOW IMSTI IMACC IDER INJR EXTR AMOT(vfem8-vfem14);

$\%$ cg\#2.c\#3\%

FG by IMKNOW* (fg1)

IMSTI IMACC IDER INJR EXTR AMOT(fg2-fg7);

[IMKNOW IMSTI IMACC IDER INJR EXTR AMOT](mmale15-mmale21);

IMKNOW IMSTI IMACC IDER INJR EXTR AMOT(vfem15-vfem21);

$\%$ cg\#2.c\#4\%

FG by IMKNOW* (fg1)

IMSTI IMACC IDER INJR EXTR AMOT(fg2-fg7);

[IMKNOW IMSTI IMACC IDER INJR EXTR AMOT](mmale22-mmale28);

IMKNOW IMSTI IMACC IDER INJR EXTR AMOT(vfem22- vfem28);

$\%$ cg\#2.c\#5\%

FG by IMKNOW* (fg1)

IMSTI IMACC IDER INJR EXTR AMOT(fg2-fg7);

[IMKNOW IMSTI IMACC IDER INJR EXTR AMOT](mmale29-mmale35);

IMKNOW IMSTI IMACC IDER INJR EXTR AMOT(vfem29- vfem35);

OUTPUT:

STDYX SAMPSTAT CINTERVAL SVALUES RESIDUAL TECH1 TECH7; 
TITLE: Mplus Input Code to Estimate a Dispersion Similarity Model for a Latent Profile Solution Without Predictors and Covariates.

! Annotations only focus on functions not previously defined.

\%OVERALL\%

FG BY IMKNOW* IMSTI IMACC IDER INJR EXTR AMOT;

FG@1; [FG@0];

c\#1 on cg\#1; c\#2 on cg\#1; c\#3 on cg\#1; c\#4 on cg\#1;

! Here, the variances are freely estimated in all profiles but constrained to equality across gender.

$\%$ cg\#1.c\#1\%

FG by IMKNOW* (fg1)

IMSTI IMACC IDER INJR EXTR AMOT(fg2-fg7);

[IMKNOW IMSTI IMACC IDER INJR EXTR AMOT](mmale1-mmale7);

IMKNOW IMSTI IMACC IDER INJR EXTR AMOT(vmale1-vmale7);

$\%$ cg\#1.c\#2\%

FG by IMKNOW* (fg1)

IMSTI IMACC IDER INJR EXTR AMOT(fg2-fg7);

[IMKNOW IMSTI IMACC IDER INJR EXTR AMOT](mmale8-mmale14);

IMKNOW IMSTI IMACC IDER INJR EXTR AMOT(vmale8-vmale14);

$\%$ cg\#1.c\#3\%

FG by IMKNOW* (fg1)

IMSTI IMACC IDER INJR EXTR AMOT(fg2-fg7);

[IMKNOW IMSTI IMACC IDER INJR EXTR AMOT](mmale15-mmale21);

IMKNOW IMSTI IMACC IDER INJR EXTR AMOT(vmale15-vmale21);

$\%$ cg\#1.c\#4\%

FG by IMKNOW* (fg1)

IMSTI IMACC IDER INJR EXTR AMOT(fg2-fg7);

[IMKNOW IMSTI IMACC IDER INJR EXTR AMOT](mmale22-mmale28);

IMKNOW IMSTI IMACC IDER INJR EXTR AMOT(vmale22-vmale28);

$\%$ cg\#1.c\#5\%

FG by IMKNOW* (fg1)

IMSTI IMACC IDER INJR EXTR AMOT(fg2-fg7):

[IMKNOW IMSTI IMACC IDER INJR EXTR AMOT](mmale29-mmale35);

IMKNOW IMSTI IMACC IDER INJR EXTR AMOT(vmale29-vmale35);

$\%$ cg\#2.c\#1\%

FG by IMKNOW* (fg1)

IMSTI IMACC IDER INJR EXTR AMOT(fg2-fg7);

[IMKNOW IMSTI IMACC IDER INJR EXTR AMOT](mmale1-mmale7);

IMKNOW IMSTI IMACC IDER INJR EXTR AMOT(vmale1-vmale7);

$\%$ cg\#2.c\#2\%

FG by IMKNOW* (fg1)

IMSTI IMACC IDER INJR EXTR AMOT(fg2-fg7); 
[IMKNOW IMSTI IMACC IDER INJR EXTR AMOT](mmale8-mmale14); IMKNOW IMSTI IMACC IDER INJR EXTR AMOT(vmale8-vmale14);

$\%$ cg\#2.c\#3\%

FG by IMKNOW* (fg1)

IMSTI IMACC IDER INJR EXTR AMOT(fg2-fg7);

[IMKNOW IMSTI IMACC IDER INJR EXTR AMOT](mmale15-mmale21);

IMKNOW IMSTI IMACC IDER INJR EXTR AMOT(vmale15-vmale21);

$\%$ cg\#2.c\#4\%

FG by IMKNOW* (fg1)

IMSTI IMACC IDER INJR EXTR AMOT(fg2-fg7):

[IMKNOW IMSTI IMACC IDER INJR EXTR AMOT](mmale22-mmale28);

IMKNOW IMSTI IMACC IDER INJR EXTR AMOT(vmale22-vmale28);

$\%$ co\#2.c\#5\%

FG by IMKNOW* (fg1)

IMSTI IMACC IDER INJR EXTR AMOT(fg2-fg7);

[IMKNOW IMSTI IMACC IDER INJR EXTR AMOT](mmale29-mmale35);

IMKNOW IMSTI IMACC IDER INJR EXTR AMOT(vmale29-vmale35);

OUTPUT:

STDYX SAMPSTAT CINTERVAL SVALUES RESIDUAL TECH1 TECH7; 
TITLE: Mplus Input Code to Estimate a Distributional Similarity Model for a Latent Profile Solution Without Predictors and

Covariates.

! Annotations only focus on functions not previously defined.

$\%$ OVERALL\%

FG BY IMKNOW* IMSTI IMACC IDER INJR EXTR AMOT;

FG@1;[FG@0];

! Taking out the following statements in the \%OVERALL\% section constrains the class sizes (class

! probabilities) to be equal across samples.

! c\#1 on cg\#1; c\#2 on cg\#1; c\#3 on cg\#1; c\#4 on cg\#1;

! Here, the variances are freely estimated in all profiles but constrained to equality across gender.

$\%$ cg\#1.c\#1\%

FG by IMKNOW* (fg1)

IMSTI IMACC IDER INJR EXTR AMOT(fg2-fg7);

[IMKNOW IMSTI IMACC IDER INJR EXTR AMOT](mmale1-mmale7);

IMKNOW IMSTI IMACC IDER INJR EXTR AMOT(vmale1-vmale7);

$\%$ cg\#1.c\#2\%

FG by IMKNOW* (fg1)

IMSTI IMACC IDER INJR EXTR AMOT(fg2-fg7):

[IMKNOW IMSTI IMACC IDER INJR EXTR AMOT](mmale8-mmale14);

IMKNOW IMSTI IMACC IDER INJR EXTR AMOT(vmale8-vmale14);

$\%$ cg\#1.c\#3\%

FG by IMKNOW* (fg1)

IMSTI IMACC IDER INJR EXTR AMOT(fg2-fg7);

[IMKNOW IMSTI IMACC IDER INJR EXTR AMOT](mmale15-mmale21);

IMKNOW IMSTI IMACC IDER INJR EXTR AMOT(vmale15-vmale21);

$\%$ cg\#1.c\#4\%

FG by IMKNOW* (fg1)

IMSTI IMACC IDER INJR EXTR AMOT(fg2-fg7);

[IMKNOW IMSTI IMACC IDER INJR EXTR AMOT](mmale22-mmale28);

IMKNOW IMSTI IMACC IDER INJR EXTR AMOT(vmale22-vmale28);

$\%$ cg\#1.c\#5\%

FG by IMKNOW* (fg1)

IMSTI IMACC IDER INJR EXTR AMOT(fg2-fg7);

[IMKNOW IMSTI IMACC IDER INJR EXTR AMOT](mmale29-mmale35);

IMKNOW IMSTI IMACC IDER INJR EXTR AMOT(vmale29-vmale35);

$\%$ cg\#2.c\#1\%

FG by IMKNOW* (fg1)

IMSTI IMACC IDER INJR EXTR AMOT(fg2-fg7);

[IMKNOW IMSTI IMACC IDER INJR EXTR AMOT](mmale1-mmale7); 
IMKNOW IMSTI IMACC IDER INJR EXTR AMOT(vmale1-vmale7); $\%$ cg\#2.c\#2\%

FG by IMKNOW* (fg1)

IMSTI IMACC IDER INJR EXTR AMOT(fg2-fg7);

[IMKNOW IMSTI IMACC IDER INJR EXTR AMOT](mmale8-mmale14);

IMKNOW IMSTI IMACC IDER INJR EXTR AMOT(vmale8-vmale14);

$\%$ cg\#2.c\#3\%

FG by IMKNOW* (fg1)

IMSTI IMACC IDER INJR EXTR AMOT(fg2-fg7);

[IMKNOW IMSTI IMACC IDER INJR EXTR AMOT](mmale15-mmale21);

IMKNOW IMSTI IMACC IDER INJR EXTR AMOT(vmale15-vmale21);

$\%$ cg\#2.c\#4\%

FG by IMKNOW* (fg1)

IMSTI IMACC IDER INJR EXTR AMOT(fg2-fg7);

[IMKNOW IMSTI IMACC IDER INJR EXTR AMOT](mmale22-mmale28);

IMKNOW IMSTI IMACC IDER INJR EXTR AMOT(vmale22-vmale28);

$\%$ cg\#2.c\#5\%

FG by IMKNOW* (fg1)

IMSTI IMACC IDER INJR EXTR AMOT(fg2-fg7);

[IMKNOW IMSTI IMACC IDER INJR EXTR AMOT](mmale29-mmale35);

IMKNOW IMSTI IMACC IDER INJR EXTR AMOT(vmale29-vmale35);

OUTPUT:

STDYX SAMPSTAT CINTERVAL SVALUES RESIDUAL TECH1 TECH7; 


\section{Mplus Input to Build the Manual 3-Step Approach}

In this study, the distribution similarity of the model was supported, which mean that we started from this model to build the manual 3-step approach prior to the integration of the covariates to ensure that the profiles themselves remained unchanged following the inclusion of the covariates. This is not always necessary, and Morin, Meyer, Creusier, \& Biétry (2016; Organizational Research Methods, 19, 231-254) provide annotated inputs scripts to guide the direct inclusion of covariates to the final multi-group latent profile solution, an approach that should always be attempted first. When this direct approach results in a switch in terms of profile definition - which was the case in the present study, then the manual 3-step approach should be retained.

Essentially, this approach consists of assigning participants to their most likely profile, while taking into account the probability that each participant has of being a member of each latent profile in the estimation of the model. To achieve this, the approach relies on the exact parameter estimates obtained from the most invariant model from the preceding sequence (here Distributional Similarity). These parameter estimates can be obtained, ready to cut and paste in a new input, while using the SVALUES function of the OUTPUT statement.

Using these exact values (these would need to be fixed with @, rather than used as start values with *), one model is estimated separately for groups (i.e. a separate model was estimated here for each gender, and age group) and used to export the class membership information to an external data file. Because one separate external data file will thus be created for each group, it will then be necessary to rely on your preferred data management package (e.g., SPSS, EXCEL) to combine the saved class membership information into a complete data set, including a clear group identification variable.

In these analyses, and all further analyses, the random start function is set to 0 , to ensure that the model converges on the same solution as retained before.

! Annotations only focus on functions not previously defined.

! This is a model estimated only for Time 1 data, using the SVALUES from the model of Distributional

! Similarity and replacing*by@.

CLASSES = c (5);

! The random start function is turned off.

Analysis:

TYPE = MIXTURE ;

ESTIMATOR $=$ MLR;

STARTS $=0$

MODEL:

\%OVERALL\%

fg BY IMKNow; fg BY IMSTI; fg BY IMACC; 
fg BY IDER; fg BY INJR; fg BY EXTR; fg BY amot;

[ c\#1@0.09309 ]; [ c\#2@0.48347 ]; [ c\#3@-0.06648 ]; [ c\#4@0.45815 ];

$\% \mathrm{C \# 1} \%$

fg BY IMKNow@0.63056 (fg1); fg BY IMSTI@0.65088 (fg2);

fg BY IMACC@0.64087 (fg3); fg BY IDER@0.17924 (fg4);

fg BY INJR@0.13154 (fg5); fg BY EXTR@0.07628 (fg6);

fg BY amot@0.01618 (fg7);

[ IMKNow@0.49434 ](mmale1); [ IMSTI@-0.31195 ] (mmale2);

[ IMACC@0.15231 ] (mmale3); [ IDER@-0.22682 ] (mmale4);

[ INJR@0.06746 ] (mmale5); [ EXTR@-0.20043 ] (mmale6);

[ amot@-0.96880 ] (mmale7); [ fg@0 ];

IMKNow@0.14447 (vmale1); IMSTI@0.36754 (vmale2);

IMACC@0.26143 (vmale3); IDER@0.52732 (vmale4);

INJR@0.57039 (vmale5); EXTR@0.75453 (vmale6);

amot@0.00343 (vmale7); fg@1;

$\% \mathrm{C \# 2} \%$

fg BY IMKNow@0.63056 (fg1); fg BY IMSTI@0.65088 (fg2);

fg BY IMACC@0.64087 (fg3); fg BY IDER@0.17924 (fg4);

fg BY INJR@0.13154 (fg5); fg BYEXTR@0.07628 (fg6);

fg BY amot@0.01618 (fg7);

[ IMKNow@0.14982 ] (mmale8); [ IMSTI@-0.22246 ] (mmale9);

[ IMACC@0.18099] (mmale10); [ IDER@-0.04261 ] (mmale11);

[ INJR@0.59827 ] (mmale12); [ EXTR@0.36692 ] (mmale13);

[ amot@-0.43390 ] (mmale14); [ fg@0 ];

IMKNow@0.09726 (vmale8); IMSTI@0.17533 (vmale9);

IMACC@0.08638 (vmale10); IDER@0.35891 (vmale11);

INJR@0.23111 (vmale12); EXTR@0.28358 (vmale13);

amot@0.15639 (vmale14); fg@1;

$\% \mathrm{C \# 3 \%}$

fg BY IMKNow@0.63056 (fg1); fg BY IMSTI@0.65088 (fg2);

fg BY IMACC@0.64087 (fg3); fg BY IDER@0.17924 (fg4);

fg BY INJR@0.13154 (fg5); fg BY EXTR@0.07628 (fg6); 
fg BY amot@0.01618 (fg7);

[ IMKNow@0.57658 ] (mmale15); [ IMSTI@-0.17109 ] (mmale16);

[ IMACC@0.63215 ] (mmale17); [ IDER@0.78720] (mmale18);

[ INJR@0.93419] (mmale19); [ EXTR@1.04222 ] (mmale20);

[ amot@-0.89382 ] (mmale21); [ fg@0 ];

IMKNow@0.20748 (vmale15); IMSTI@0.38178 (vmale16);

IMACC@0.14594 (vmale17); IDER@0.06393 (vmale18);

INJR@0.22515 (vmale19); EXTR@0.02866 (vmale20);

amot@0.00602 (vmale21); fg@1;

$\% \mathrm{C} \# 4 \%$

fg BY IMKNow@0.63056 (fg1); fg BY IMSTI@0.65088 (fg2);

fg BY IMACC@0.64087 (fg3); fg BY IDER@0.17924 (fg4);

fg BY INJR@0.13154 (fg5); fg BY EXTR@0.07628 (fg6);

fg BY amot@0.01618 (fg7);

[ IMKNow@-0.76964 ] (mmale22); [ IMSTI@-0.11838 ] (mmale23);

[ IMACC@-0.55830 ] (mmale24); [ IDER@-0.63603 ] (mmale25);

[ INJR@-0.36029 ] (mmale26); [ EXTR@-0.80624 ] (mmale27);

[ amot@0.67860 ] (mmale28); [ fg@0 ];

IMKNow@0.38756 (vmale22); IMSTI@0.33262 (vmale23);

IMACC@0.34946 (vmale24); IDER@1.10926 (vmale25);

INJR@0.53180 (vmale26); EXTR@0.76665 (vmale27);

amot@0.31889 (vmale28); fg@1;

$\% \mathrm{C \# 5 \%}$

fg BY IMKNow@0.63056 (fg1); fg BY IMSTI@0.65088 (fg2);

fg BY IMACC@0.64087 (fg3); fg BY IDER@0.17924 (fg4);

fg BY INJR@0.13154 (fg5); fg BY EXTR@0.07628 (fg6);

fg BY amot@0.01618 (fg7);

[ IMKNow@-0.10818 ] (mmale29); [ IMSTI@1.05040] (mmale30);

[ IMACC@-0.16937 ] (mmale31); [ IDER@0.58715 ] (mmale32);

[ INJR@-1.34869 ] (mmale33); [ EXTR@-0.07544 ] (mmale34);

[ amot@1.53027] (mmale35); [ fg@0 ];

IMKNow@0.27725 (vmale29); IMSTI@0.10598 (vmale30); 
IMACC@0.38452 (vmale31); IDER@0.31324 (vmale32);

INJR@0.05684 (vmale33); EXTR@0.44880 (vmale34);

amot@0.05338 (vmale35); fg@1;

! The SAVEDATA function is used to export the class membership information to an external data file,

! that is labelled cmale.dat here.

SAVEDATA: file $=$ cmale.dat; save $=$ cprob;

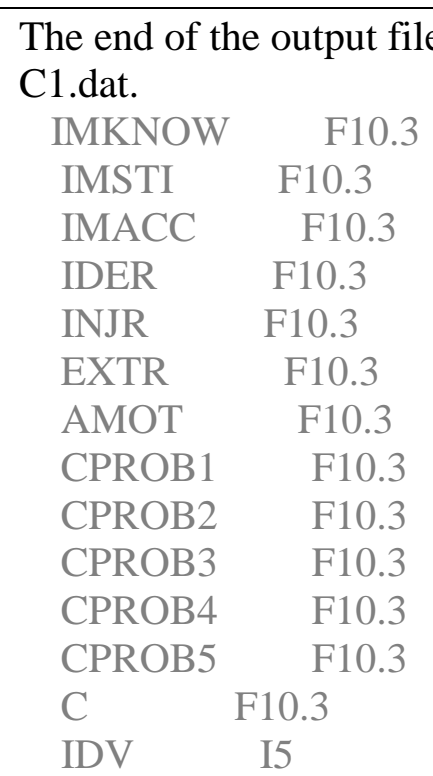

The variable $\mathrm{C}$ refers to the most likely class membership of each participant. This is the variable that needs to be brought back into the main data file, where it is relabeled CL to avoid confusion with the way Mplus labels latent profiles. The same process is then repeated for female participants (and all other groups, whenever necessary). Using this information (CL), it then becomes possible to build an input file that will automatically, and systematically, result in the same classification of participants, while taking into account the class probabilities. More precisely, these variables are used directly in the model estimation process to force the assignment of participants into each profile.

Before doing so, however, we need further information from the outputs associated with the constrained estimations of the male and female models. Just before the MODEL RESULTS section, at the end of the MODEL FIT INFORMATION section, there is a Table of logits: 


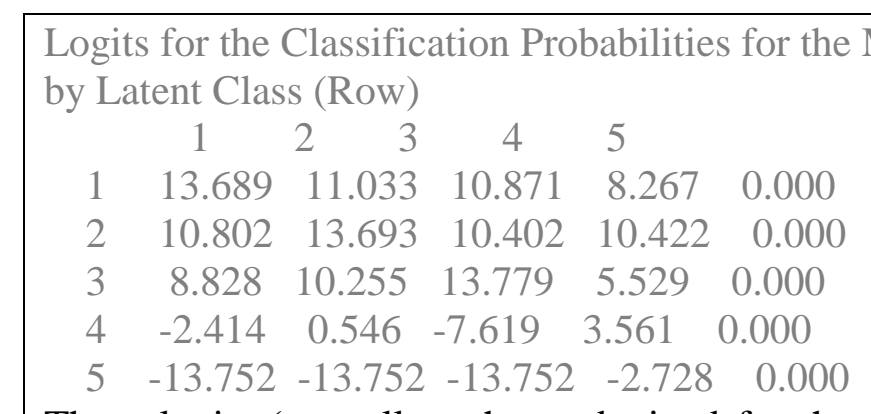

These logits (as well as those obtained for the female participants) reflect the class probabilities of assignments of the participants into the various profiles and are used in the input to "correct" the class assignment of the participants into each profile while taking into account their probability of membership into the other profiles. This process corresponds to modeling class assignment while controlling for the imprecise nature of this assignment, a process akin to controlling for measurement errors.

On next page is the input used to provide an exact replication of the model of Distributional Similarity. 
! The only variable that needs to be used is $C L$, which is nominal in nature and reflect the

! most likely profile membership of each participant.

!Then, in the class-specific statement, this membership is "corrected" using the logit values (@).

\section{USEVARIABLES = CL;}

nominal $=\mathrm{CL}$;

missing are all *;

IDVARIABLE = ID;

KNOWNCLASS $=$ CG $($ GENDER $=1$ GENDER $=2)$

CLASSES = CG (2) C (5);

Analysis:

TYPE = MIXTURE $;$ ESTIMATOR = MLR; PROCESS = 3;

STARTS $=0$

MODEL:

$\%$ OVERALL $\%$

$\%$ cg\#1.c\#1\%

[Cl\#1@13.689 ]; [Cl\#2@11.033];

[Cl\#3@10.871]; [Cl\#4@8.267];

$\%$ cg\#1.c\#2\%

[Cl\#1@10.802 ]; [Cl\#2@13.693];

[Cl\#3@10.402]; [Cl\#4@ 10.422];

$\%$ cg\#1.c\#3\%

[Cl\#1@8.828 ]; [Cl\#2@10.255];

[Cl\#3@13.779]; [Cl\#4@5.529];

$\%$ cg\#1.c\#4\%

[Cl\#1@-2.414]; [Cl\#2@0.546];

[Cl\#3@-7.619]; [Cl\#4@3.561];

$\%$ cg\#1.c\#5\%

[Cl\#1@-13.752]; [Cl\#2@-13.752];

[Cl\#3@-13.752]; [Cl\#4@-2.768];

$\%$ cg\#2.c\#1\%

[Cl\#1@13.705 ]; [Cl\#2@11.110]; 
[Cl\#3@10.540]; [Cl\#4@4.061];

$\%$ cg\#2.c\#2\%

[Cl\#1@11.502]; [Cl\#2@13.653];

[Cl\#3@10.199]; [Cl\#4@10.097];

$\%$ cg\#2.c\#3\%

[Cl\#1@9.221]; [Cl\#2@8.836];

[Cl\#3@13.798]; [Cl\#4@0];

$\%$ cg\#2.c\#4\%

[Cl\#1@-1.802 ]; [Cl\#2@0.295];

[Cl\#3@-5.048]; [Cl\#4@3.106];

$\%$ cg\#2.c\#5\%

[Cl\#1@-13.783]; [Cl\#2@-13.783];

[Cl\#3@-13.783]; [Cl\#4@-3.399];

This model is then used to incorporate predictors and covariates, as shown in the following sections. 
TITLE: Mplus Input Code to Estimate a Latent Profile Solution With Predictors Effects Freely Estimated Across Samples Using the Manual 3-Step Approach.

! Annotations only focus on functions not previously defined.

DATA: FILE IS FSCORES.dat;

VARIABLE:

NAMES = ID GENDER AGEG3 IMKNOW IMSTI IMACC IDER INJR EXTR AMOT

VITAL MINVOLV MAUTOSUP MWARM FINVOLV PAUTOSUP FWARM CL;

USEVARIABLES = CL MINVOLV MAUTOSUP MWARM;

MISSING ARE ALL *;

NOMINAL $=$ CL;

IDVARIABLE $=$ ID;

KNOWNCLASS $=$ CG $($ GENDER $=1$ GENDER $=2)$;

CLASSES = CG (2) C (5);

Analysis:

TYPE = MIXTURE; ESTIMATOR = MLR; PROCESS = 3;

STARTS $=0$

! The following are required to activate FIML when missing data are present on the predictors ALGORITHM=INTEGRATION;

INTEGRATION = montecarlo;

MODEL:

$\%$ OVERALL\%

! For identification purposes, the effects of the predictors on class membership needs to be

! constrained to zero in the overall statement, in order to be freely estimated in all groups. c\#1-c\#4 ON MINVOLV@0 MAUTOSUP@0 MWARM@0 ;

! The following are required to activate FIML when missing data are present on the predictors MINVOLV MAUTOSUP MWARM;

! The following are required to activate FIML when missing data are present on the predictors $\%$ cg\#1.c\#1\%

[Cl\#1@13.689 ]; [Cl\#2@11.033]; [Cl\#3@10.871]; [Cl\#4@ @ 8.267];

$\%$ cg\#1.c\#2\%

[Cl\#1@10.802 ]; [Cl\#2@13.693]; [Cl\#3@10.402]; [Cl\#4@10.422];

$\%$ cg\#1.c\#3\% 
[Cl\#1@8.828 ]; [Cl\#2@10.255]; [Cl\#3@13.779]; [Cl\#4@ 5.529];

$\%$ cg\#1.c\#4\%

[Cl\#1@-2.414]; [Cl\#2@0.546]; [Cl\#3@-7.619]; [Cl\#4@ 3.561];

$\%$ cg\#1.c\#5\%

[Cl\#1@-13.752]; [Cl\#2@-13.752]; [Cl\#3@-13.752]; [Cl\#4@-2.768];

$\%$ cg\#2.c\#1\%

[Cl\#1@13.705 ]; [Cl\#2@11.110]; [Cl\#3@10.540]; [Cl\#4@4.061];

$\%$ cg\#2.c\#2\%

[Cl\#1@11.502]; [Cl\#2@ 13.653]; [Cl\#3@10.199]; [Cl\#4@ 10.097];

$\%$ cg\#2.c\#3\%

[Cl\#1@9.221]; [Cl\#2@8.836]; [Cl\#3@13.798]; [Cl\#4@0];

$\%$ cg\#2.c\#4\%

[Cl\#1@-1.802 ]; [Cl\#2@0.295]; [Cl\#3@-5.048]; [Cl\#4@3.106];

$\%$ cg\#2.c\#5\%

[Cl\#1@-13.783]; [Cl\#2@-13.783]; [Cl\#3@-13.783]; [Cl\#4@-3.399];

! One additional section is needed to allow the predictions to differ across groups (MODEL CG)

MODEL cg:

$\%$ cg\#1\%

c\#1-c\#4 ON MINVOLV MAUTOSUP MWARM ;

MINVOLV MAUTOSUP MWARM ;

$\%$ cg\# $2 \%$

c\#1-c\#4 ON MINVOLV MAUTOSUP MWARM ;

MINVOLV MAUTOSUP MWARM ; 
TITLE: Mplus Input Code to Estimate a Predictive Similarity Latent Profile Solution Using the Manual 3-Step Approach.

! Annotations only focus on functions not previously defined.

MODEL:

\%OVERALL\%

! Here, the effects of the predictors on class membership is freely estimated in the overall statement.

! By default, this is estimated as equal across groups

c\#1-c\#4 ON MINVOLV MAUTOSUP MWARM;

MINVOLV MAUTOSUP MWARM;

$\%$ cg\#1.c\#1\%

[Cl\#1@13.689 ]; [Cl\#2@11.033]; [Cl\#3@10.871]; [Cl\#4@8.267];

$\%$ cg\#1.c\#2\%

[Cl\#1@10.802 ]; [Cl\#2@ 13.693]; [Cl\#3@10.402]; [Cl\#4@ 10.422];

\%cg\#1.c\#3\%

[Cl\#1@8.828 ]; [Cl\#2@10.255]; [Cl\#3@13.779]; [Cl\#4@5.529];

$\%$ cg\#1.c\#4\%

[Cl\#1@-2.414]; [Cl\#2@0.546]; [Cl\#3@-7.619]; [Cl\#4@3.561];

\%cg\#1.c\#5\%

[Cl\#1@-13.752]; [Cl\#2@-13.752]; [Cl\#3@-13.752]; [Cl\#4@-2.768];

$\%$ cg\#2.c\#1\%

[Cl\#1@13.705 ]; [Cl\#2@11.110]; [Cl\#3@ 10.540]; [Cl\#4@4.061];

$\%$ cg\#2.c\#2\%

[Cl\#1@ 11.502]; [Cl\#2@ 13.653]; [Cl\#3@ 10.199]; [Cl\#4@ 10.097];

$\%$ cg\#2.c\#3\%

[Cl\#1@9.221]; [Cl\#2@8.836]; [Cl\#3@13.798]; [Cl\#4@0];

$\%$ cg\#2.c\#4\%

[Cl\#1@-1.802 ]; [Cl\#2@0.295]; [Cl\#3@-5.048]; [Cl\#4@3.106];

$\%$ cg\#2.c\#5\%

[Cl\#1@-13.783]; [Cl\#2@-13.783]; [Cl\#3@-13.783]; [Cl\#4@-3.399];

!There is no need for a $C G$ section. 
TITLE: Mplus Input Code to Estimate a Latent Profile Solution With Covariate Levels Freely Estimated Across Samples Using the Manual 3-Step Approach.

! Annotations only focus on functions not previously defined.

DATA: FILE IS FSCORES.dat;

VARIABLE:

NAMES = ID GENDER AGEG3 IMKNOW IMSTI IMACC IDER INJR EXTR AMOT

VITAL MINVOLV MAUTOSUP MWARM FINVOLV PAUTOSUP FWARM CL;

USEVARIABLES = CL VITAL;

MISSING ARE ALL *;

NOMINAL $=$ CL;

IDVARIABLE = ID;

KNOWNCLASS $=$ CG $($ GENDER $=1$ GENDER $=2)$;

CLASSES $=$ CG (2) C (5);

Analysis:

TYPE = MIXTURE; ESTIMATOR = MLR; PROCESS = 3;

STARTS $=0$

! Statements related to the covariates are added in each specific profile/group combination and labels

! are used to specify that mean levels on these covariates are freely estimated in all profiles/groups

MODEL:

\%OVERALL\%

$\%$ cg\#1.c\#1\%

[Cl\#1@13.689 ]; [Cl\#2@11.033]; [Cl\#3@10.871]; [Cl\#4@8.267];

[Vital] (o1);

$\%$ cg\#1.c\#2\%

[Cl\#1@10.802 ]; [Cl\#2@13.693]; [Cl\#3@ 10.402]; [Cl\#4@ 10.422];

[Vital] (o2);

$\%$ cg\#1.c\#3\%

[Cl\#1@8.828 ]; [Cl\#2@ 10.255]; [Cl\#3@ 13.779]; [Cl\#4@ 5.529];

[Vital] (o3);

$\%$ cg\#1.c\#4\%

[Cl\#1@-2.414]; [Cl\#2@0.546]; [Cl\#3@-7.619]; [Cl\#4@3.561];

[Vital] (o4); 
$\%$ cg\#1.c\#5\%

[Cl\#1@-13.752]; [Cl\#2@-13.752]; [Cl\#3@-13.752]; [Cl\#4@-2.768];

[Vital] (o5);

$\%$ cg\#2.c\#1\%

[Cl\#1@13.705 ]; [Cl\#2@11.110]; [Cl\#3@10.540]; [Cl\#4@4.061];

[Vital] (oo1);

$\%$ cg\#2.c\#2\%

[Cl\#1@11.502]; [Cl\#2@13.653]; [Cl\#3@10.199]; [Cl\#4@10.097];

[Vital] (oo2);

$\%$ cg\#2.c\#3\%

[Cl\#1@9.221]; [Cl\#2@8.836]; [Cl\#3@13.798]; [Cl\#4@0];

[Vital] (oo3);

$\%$ cg\#2.c\#4\%

[Cl\#1@-1.802 ]; [Cl\#2@0.295]; [Cl\#3@-5.048]; [Cl\#4@3.106];

[Vital] (oo4);

$\%$ cg\#2.c\#5\%

[Cl\#1@-13.783]; [Cl\#2@-13.783]; [Cl\#3@-13.783]; [Cl\#4@-3.399];

[Vital] (oo5); 
TITLE: Mplus Input Code to Estimate an Explanatory Similarity Latent Profile Solution Using the Manual 3-Step Approach.

! Annotations only focus on functions not previously defined.

DATA: FILE IS FSCORES.dat;

VARIABLE:

NAMES = ID GENDER AGEG3 IMKNOW IMSTI IMACC IDER INJR EXTR AMOT

VITAL MINVOLV MAUTOSUP MWARM FINVOLV PAUTOSUP FWARM CL;

USEVARIABLES = CL VITAL;

MISSING ARE ALL *;

NOMINAL $=$ CL;

IDVARIABLE $=$ ID;

KNOWNCLASS $=$ CG $($ GENDER $=1$ GENDER $=2)$;

CLASSES = CG (2) C (5);

Analysis:

TYPE $=$ MIXTURE; ESTIMATOR = MLR; PROCESS $=3$;

STARTS $=0$

! Statements related to the covariates are added in each specific profile/group combination and labels

! are used to specify that mean levels on these covariates are similar across groups

MODEL:

$\%$ OVERALL \%

$\%$ cg\#1.c\#1\%

[Cl\#1@13.689]; [Cl\#2@11.033]; [Cl\#3@10.871]; [Cl\#4@8.267];

[Vital] (o1);

$\%$ cg\#1.c\#2\%

[Cl\#1@10.802 ]; [Cl\#2@13.693]; [Cl\#3@10.402]; [Cl\#4@10.422];

[Vital] (o2);

$\%$ cg\#1.c\#3\%

[Cl\#1@8.828 ]; [Cl\#2@10.255]; [Cl\#3@13.779]; [Cl\#4@ 5.529];

[Vital] (o3);

$\%$ cg\#1.c\#4\%

[Cl\#1@-2.414]; [Cl\#2@0.546]; [Cl\#3@-7.619]; [Cl\#4@3.561];

[Vital] (o4);

$\%$ cg\#1.c\#5\% 
[Cl\#1@-13.752]; [Cl\#2@-13.752]; [Cl\#3@-13.752]; [Cl\#4@-2.768];

[Vital] (05);

$\%$ cg\#2.c\#1\%

[Cl\#1@13.705 ]; [Cl\#2@11.110]; [Cl\#3@10.540]; [Cl\#4@4.061];

[Vital] (o1);

$\%$ cg\#2.c\#2\%

[Cl\#1@11.502]; [Cl\#2@13.653]; [Cl\#3@10.199]; [Cl\#4@10.097];

[Vital] (o2);

$\%$ cg\#2.c\#3\%

[Cl\#1@9.221]; [Cl\#2@8.836]; [Cl\#3@13.798]; [Cl\#4@0];

[Vital] (o3);

$\%$ cg\#2.c\#4\%

[Cl\#1@-1.802 ]; [Cl\#2@0.295]; [Cl\#3@-5.048]; [Cl\#4@3.106];

[Vital] (o4);

$\%$ cg\#2.c\#5\%

[Cl\#1@-13.783]; [Cl\#2@-13.783]; [Cl\#3@-13.783]; [Cl\#4@-3.399];

[Vital] (o5); 


\section{MODEL CONSTRAINT:}

! New parameters are created using this function and reflect pairwise mean differences between

! profiles. So the first of those (yo12) reflect the differences between the means of profiles 1 and 2.

! This will be included in the outputs as new parameters reflecting the significance of

! the differences between the means, without those parameters having an impact on the model.

NEW (yo12);

yo12 = o1-o2;

NEW (yo13);

yo13 = o1-o3;

NEW (yo14);

yo14 = o1-o4;

NEW (yo15);

yo15 = o1-o5;

NEW (yo23);

yo23 = o2-o3;

NEW (yo24);

yo24 = o2-o4;

NEW (yo25);

yo25 = o2-o5;

NEW (yo34);

yo34 = o3-o4;

NEW (yo35);

yo35 = o3-o5;

NEW (yo45);

yo45 = o4-o5; 